\title{
Enhanced recovery after gynecologic surgery
}

Citation for published version (APA):

de Groot, J. J. A. (2020). Enhanced recovery after gynecologic surgery: A focus on implementation challenges. [Doctoral Thesis, Maastricht University]. Maastricht University. https://doi.org/10.26481/dis.20200408jg

Document status and date:

Published: 01/01/2020

DOI:

10.26481/dis.20200408jg

Document Version:

Publisher's PDF, also known as Version of record

\section{Please check the document version of this publication:}

- A submitted manuscript is the version of the article upon submission and before peer-review. There can be important differences between the submitted version and the official published version of record.

People interested in the research are advised to contact the author for the final version of the publication, or visit the DOI to the publisher's website.

- The final author version and the galley proof are versions of the publication after peer review.

- The final published version features the final layout of the paper including the volume, issue and page numbers.

Link to publication

\footnotetext{
General rights rights.

- You may freely distribute the URL identifying the publication in the public portal. please follow below link for the End User Agreement:

www.umlib.nl/taverne-license

Take down policy

If you believe that this document breaches copyright please contact us at:

repository@maastrichtuniversity.nl

providing details and we will investigate your claim.
}

Copyright and moral rights for the publications made accessible in the public portal are retained by the authors and/or other copyright owners and it is a condition of accessing publications that users recognise and abide by the legal requirements associated with these

- Users may download and print one copy of any publication from the public portal for the purpose of private study or research.

- You may not further distribute the material or use it for any profit-making activity or commercial gain

If the publication is distributed under the terms of Article $25 \mathrm{fa}$ of the Dutch Copyright Act, indicated by the "Taverne" license above, 
Enhanced recovery after gynecologic surgery

A focus on implementation challenges

Jeanny de Groot 
The research presented in this thesis was conducted at CAPHRI Care and Public Health Research Institute, Department Family Medicine, of Maastricht University. CAPHRI participates in the Netherlands School of Public Health and Care Research CaRe.

The studies in this thesis were supported by a grant from the Netherlands Organization for Health Research and Development (ZonMw).

For most articles published, the copyright has been transferred to the respective publisher. No part of this thesis may be reproduced in any form or by any means without written permission from the author or, when appropriate, from the publisher.

(C) Jeanny de Groot, Maastricht 2020

Cover design, layout and print: Optima Grafische Communicatie

ISBN 978-94-6361-401-6 


\section{Enhanced recovery after gynecologic surgery A focus on implementation challenges}

\section{PROEFSCHRIFT}

Ter verkrijging van de graad van doctor aan de Universiteit Maastricht, op gezag van Rector Magnificus, Prof. dr. R.M. Letschert, volgens het besluit van het College van Decanen, in het openbaar te verdedigen op woensdag 8 april 2020 om 16 h00

$$
\text { door }
$$

Jeanny Johanna Anna de Groot 


\section{Promotor}

Prof. dr. T. van der Weijden

\section{Copromotores}

Dr. J.M.C. Maessen

Dr. B.F.M. Slangen

\section{Beoordelingscommissie}

Prof. dr. C.G. Faber (voorzitter)

Dr. L. van Bodegom - Vos (Leids Universitair Medisch Centrum)

Prof. dr. T. van Gorp (Universitair Ziekenhuis Leuven, België)

Prof. dr. I.H.J.Th. de Hingh

Prof. dr. G.G. van Merode 


\section{Table of contents}

$\begin{array}{lll}\text { Chapter } 1 & \text { General introduction } & 7\end{array}$

Chapter 2 Enhanced recovery pathways in abdominal gynecologic surgery: 21 a systematic review and meta-analysis

Chapter 3 Interdepartmental spread of innovations: a multicenter study 53 of the enhanced recovery after surgery program

Chapter 4 Diffusion of Enhanced Recovery principles in gynecologic on69 cology surgery: is active implementation still necessary?

Chapter 5 A stepped strategy that aims at the nationwide implementation of the Enhanced Recovery After Surgery program in major gynecologic surgery: study protocol of a cluster randomized controlled trial

Chapter 6 Evaluating a tailored stepped implementation strategy on the improvement of perioperative care in gynecologic oncology. A randomized clinical trial.

Chapter 7 Expanding the scope of quality improvement strategies: a multiphase observational study to increase insight into nonparticipants

Chapter 8 General discussion

Chapter 9 Summary

Chapter 10 Nederlandse samenvatting

Chapter 11 Epilogue 179

Valorisation

Contributing centers

Dankwoord

Curriculum Vitae 189

List of publications 



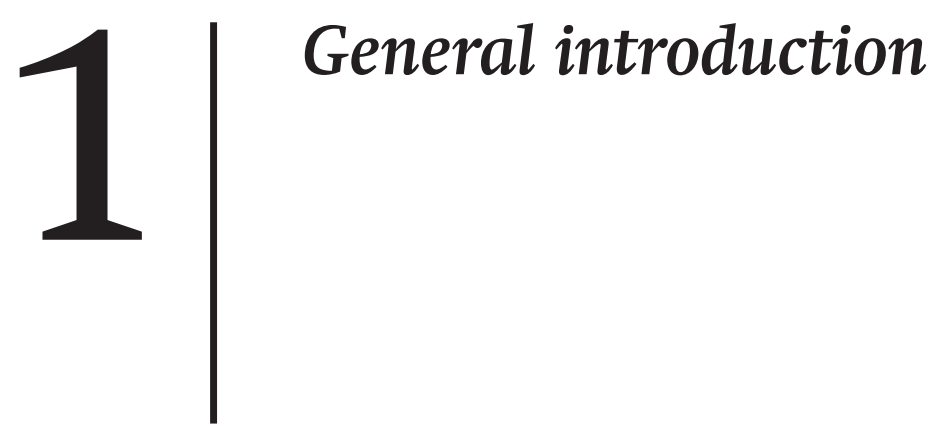



"The immediate challenge to improving the quality of surgical care is not discovering new knowledge, but rather how to integrate what we already know into practice"

- Urbach et al [1]-

Implementation science focuses on challenges associated with the systematic uptake of research findings into routine professional practice [2]. The statement of Urbach and colleagues [1] characterizes one of the major problems faced in health care today. The substantial gap between best practices and current care around surgical procedures is the main focus of this thesis. It aims to provide insight into the challenges of implementation in actual practice, with a focus on perioperative care around gynecologic surgery in the Netherlands. The design and effectiveness of an empirically driven and theoretically based implementation strategy is described and analyzed in order to provide a more efficient implementation. This introductory chapter captures background information on knowledge translation and the enhanced recovery after surgery program with a summary on the state of art at the start of this thesis in 2013. The introduction ends with a short outline of the thesis content.

\section{The challenges of using evidence based practice}

Key to improving quality and effectiveness of health care is using the knowledge already gained. Evidence based practice is not just determined by increasing or validating medical knowledge. It also depends on the application of this evidence, ideally synthesized and interpreted in clinical practice guidelines, in specific clinical settings $[3,4]$. By incorporating the best available evidence in daily practice, it is aimed to improve patient outcomes in an efficient manner to deliver the highest quality of care possible. New clinical evidence has become more easily accessible during the past quarter century, with the number of newly published articles increasing substantially each year. The annual number of indexed biomedical articles in Embase (Excerpta Medica Database) has reached the one million mark. This results in a significant source of medical information which is important to guide evidence-based daily practice.

Although much progress on evidence-based medicine has been made, we have become increasingly aware of the challenges induced by this major growth in available literature. This results at best in a reduced half-life of the available knowledge, or even in unused knowledge. The growing body of evidence emerges a challenge, as it is well known that translation of established research evidence into daily practice is already often delayed and incomplete $[5,6]$. This phenomenon has been identified as the evidence-practice gap and may last for several years. Apart from the overwhelming annual amount of evidence produced, multiple underlying reasons can be determined to clarify this evidence-practice gap [7]. 
Research findings of mostly randomized controlled trials and systematic literature reviews are thoroughly captured into (discipline-specific) national guidelines. Those guidelines are subsequently translated into local hospital protocols which clinicians use in their decision-making for the individual patient [8]. Although clinical practice guidelines are helpful in itself for the adoption of research findings [9], the development procedures of guidelines take time and result in an additional delay. Furthermore, studies have shown that actual adherence to clinical guidelines, once disseminated and accessible, is limited $[10,11]$. Adherence is often much lower than the desirable benchmarks and is often overestimated by healthcare professionals. Outcome measures of studies used in guidelines are not always applicable to specific clinical situations and the complex structure of healthcare systems may also complicate optimal translation of new knowledge in actual practice.

Patients are therefore not only receiving suboptimal health care, but are also exposed to potential unnecessary, or even harmful, elements of care. It is estimated that those percentages are at least $30-40 \%$ and $20-25 \%$, respectively $[6,12-14]$. In addition, this will lead to a waste of valuable healthcare resources. Without substantial changes, these challenges, resulting from the growing complexity of evidence-based health care, will make it even more difficult to apply medical knowledge efficiently.

\section{Knowledge translation}

The effect of new clinical evidence could be maximized by optimizing the knowledge translation processes in which healthcare systems use the available evidence. [3, 15] Change management has become increasingly important on system level. Quality improvement interventions are needed to change daily practice and can be defined as 'ensuring that stakeholders are aware of and use research evidence to inform their health and healthcare decision-making' [16]. These efforts can be described differently and in literature overlapping terms are used. Grimshaw et al. [16] provided an overview including terms as knowledge translation, knowledge utilization, knowledge transfer, quality improvement, innovation diffusion, implementation, research utilization, and quality assurance.

The process of change is multi-factorial and is influenced by individual factors related to the end users of medical knowledge, social factors related to e.g. teams of professionals, and factors at the system level [17]. As a result, many theories of change are involved. Rogers' diffusion of innovation theory [18] is one of the most referred theories and plays a key role throughout this thesis. The theory includes factors that influence adoption and describes different adopter categories of a target population: innovators, early adopters, early majority, late majority, and laggards [18]. It is thought that the so-called innovators start adopting the innovation, with 
the early majority, late majority and laggards to follow consecutively. This framework enables the conceptualization of different types of social change.

When different healthcare professionals, disciplines and locations are involved, the change process will be even more complex as needs, priorities and abilities to change may vary between subgroups, and will require more effort. An efficient, theoretically based implementation strategy that is adaptable to the varying circumstances is necessary. The Cochrane Effective Practice and Organization of Care (EPOC) group describes a taxonomy of different quality improvement interventions aimed at professional, patient, organizational, financial, and regulatory level [19, 20]. These interventions may for example be printed educational materials, educational meetings, local opinion leaders, audit and feedback, clinical multidisciplinary teams, or changing continuity of care by redesigning the care pathway. Multifaceted strategies, combining several quality improvement interventions, have demonstrated that healthcare can be partially improved, however studies showed mixed results [21-23]. Overall, effects of quality improvement programs may be limited, but relevant, and improvement is not directly related to the number of components used [16]. Grimshaw et al. reported an overall absolute effect size of around 10\% [12, 22]. Tailored interventions, which are characterized by addressing previously identified barriers and facilitators to change, are thought to be superior in the improvement of clinical practice [24]. Although the need for an adaptive strategy is evident, overall effectiveness varies.

\section{Multi-organizational change}

In their systematic literature review, Nadeem et al. [25] tried to describe the specific components and impact of twenty multi-organizational quality improvement efforts in health care. The included collaboratives of multiple organizations mainly utilized the Breakthrough Series model, a short-term cyclic learning system developed by the Institute for Healthcare Improvement, as a tool for quality improvement [26]. The Breakthrough Series was published in 1998 and is worldwide one of the most used and best documented model for quality improvement [27]. Although there are many variations of this model, the basic concepts have remained unchanged. Interdisciplinary improvement teams conduct serial plan-do-study-act cycles to improve specific care. By using a plan-do-study-act approach, refinements can be made after each cycle. The teams participate in three learning sessions and receive training from an expert team. Nadeem et al. [25] showed that reporting of specific components in quality improvement collaboratives was often incomplete, hindering further analysis of the impact on both patient and provider level. Only three studies involved an active comparison condition in an experimental design, and evaluation showed that those studies were less likely to find any effect at all [25]. 
In conclusion, implementation of quality improvement initiatives on large scale remains suboptimal and evidence about their impact could be improved [27]. Research on how to optimize resources is warranted to deliver an optimal effect of quality improvement interventions with minimal investments. More primary data are needed to define the ideal implementation strategies for improving quality of care that can be modified and adapted to specific healthcare settings [22]. Adapting implementation strategies to a more efficient and tailored model might be important for effective large scale implementation in the future.

\section{Updating the quality of perioperative care}

The quality improvement of perioperative care was particularly initiated by Kehlet in 1997, in patients undergoing elective colonic surgery [28]. He proved that the perioperative stress response could be minimized by controlling physiology. A multimodal approach was designed to prevent cellular dysfunction, changes in metabolism, and disturbances in homeostasis [29, 30]. Innovations in the field of surgery and anesthesiology, such as minimally invasive surgery and regional analgesia, contributed to this progress. As a consequence, functional recovery accelerated resulting in improved postoperative outcomes [31]. After initial criticism, the positive outcomes of his approach in patients undergoing elective colonic surgery were widely acknowledged. Four other hospitals of different countries joined his initiative and founded the Enhanced Recovery After Surgery (ERAS) collaboration in 2001. The large unwarranted practice variation in perioperative care between their hospitals was addressed [32] and the fast track approach evolved further towards a more extensive perioperative program for colorectal surgery patients [33]. The elements included in the ERAS program are evidence-based and have a synergistic effect on outcome measures as the improvements seen were larger than what could be expected from the single elements. The ERAS program reduced the length of hospital stay with approximately $30 \%$ and the percentage of major complications was almost halved compared to traditional care [34, 35]. Studies have shown that overall hospital costs could be reduced by the implementation of the program and patient satisfaction could be improved, without an increase in workload [36, 37]. These positive results have shown to be transferable to other surgical disciplines, inducing the spread of ERAS principles by variations of the protocol [38]. On top of the ongoing aim to improve postoperative recovery and the development of perioperative care, optimizing implementation and sustainability of the program has gained attention nowadays.

In the Netherlands, the ERAS program was implemented in colonic surgery in 33 hospitals by using a Breakthrough Series model between 2005 and 2009 [39]. Participating hospitals followed a one-year implementation project consisting of 
three general learning sessions. Their performance was monitored and feedback was provided by an expert team under guidance of the Dutch Institute for Health Care Improvement (CBO). These hospitals were able to reduce length of stay and improved their clinical practice in elective colonic surgery [39]. A follow-up study performed three to six years after initial implementation showed that the improvements made were not fully sustained for a longer period of time [40].

More evidence about the value of ERAS for gynecologic surgery has become available during the last decade [41-45]. Several preoperative, intra-operative, and postoperative recommendations were studied. The main recommendations during these phases are described in Table 1. Compliance of both patients and professionals to the single protocol elements is essential to ensure successful outcomes [46]. Fast recovery after oncologic surgery in gynecology is particularly important to improve oncologic outcomes by enabling the urgent start of adjuvant treatment. Furthermore, implementation of the ERAS guideline leads to standardization of clinical practice. This contributes to the prerequisite that patients should all be treated in the same way which is important for the comparison of specific outcome measures for other multicenter trials. The ongoing hospital consolidation and centralization of clinical care in the Netherlands, resulting in a higher patients load per hospital, is an additional argument for the adoption of ERAS principles within gynecology to endeavor fast recovery and hospital discharge. In conclusion, several arguments strengthen the fact that ERAS nowadays should be standard care within gynecologic surgery.

Table 1 Main ERAS recommendations

\begin{tabular}{|c|c|}
\hline \multirow[t]{5}{*}{ Preoperative } & Preadmission counselling \\
\hline & Use of carbohydrate load \\
\hline & No prolonged fasting \\
\hline & No bowel preparation \\
\hline & No premedication \\
\hline \multirow[t]{6}{*}{ Intra-operative } & Use of local analgesia \\
\hline & Avoidance of drains \\
\hline & Avoidance of intravenous fluid overload \\
\hline & Maintenance of normothermia \\
\hline & No nasogastric tubes \\
\hline & Prevention of nausea and vomiting \\
\hline \multirow[t]{7}{*}{ Postoperative } & Non-opioid oral analgesia \\
\hline & Early discontinuation of intravenous fluids \\
\hline & Early removal of urinary catheter \\
\hline & Early oral nutrition \\
\hline & Early ambulation \\
\hline & Stimulation of gut motility (standard laxatives, chewing gum) \\
\hline & Audit of compliance and outcomes \\
\hline
\end{tabular}

ERAS, enhanced recovery after surgery 


\section{OBJECTIVES AND OUTLINE}

Although ERAS pathways are based on single evidence-based elements [33], overall effects in abdominal gynecologic surgery remain undetermined. However, the ERAS program can still be regarded as the best available evidence-based practice in perioperative settings. Optimal perioperative care represents a team-based effort. The multimodal approach with interacting elements challenges efficient and sustainable implementation. The Breakthrough Series model is the most commonly used collaborative for quality improvement on a large scale and has proven to be effective for the implementation of ERAS [39]. However, underlying evidence of this collaborative is limited and the model with generic sessions for the varying settings is time consuming [27, 47], questioning the cost-effectiveness of the Breakthrough Series approach. The ideal multi-organizational implementation strategy should be based on the existing evidence, could be adapted to varying clinical settings, and would be able to deliver an optimal effect at the lowest possible costs.

This thesis aims to provide further information on the process of implementation by exploring the adoption of the ERAS guideline in gynecologic surgery in the Netherlands. It takes up the challenge to define a preferred efficient strategy for the nationwide dissemination of a multimodal perioperative improvement program. The following research questions were defined to provide insight into the context, process and effectiveness of the nationwide implementation of ERAS in actual perioperative practice around gynecologic surgical procedures:

- Which ERAS elements are already used in gynecologic surgery and what is known about their effect on postoperative outcomes? (chapter 2)

- What is the current status of perioperative care in gynecologic surgery in the Netherlands and how is the adoption of ERAS elements in actual practice? (chapter 3)

- How do spontaneous diffusion and structured implementation of ERAS in other departments impact implementation of ERAS in gynecologic surgery and what is their relation to patient's outcomes? (chapter 3,4)

- Does an efficient, tailored model to implement ERAS across multiple hospitals make large scale implementation strategies more effective and to what degree do intervention fidelity and contextual factors influence implementation? (chapter $5,6,7)$

These questions are addressed in six studies, comprising a systematic literature review, retrospective pre-post studies, and a cluster randomized controlled trial. The results of these studies are presented in the current thesis. 
Chapter 2 aims to provide a systematic overview of the available literature on ERAS in abdominal gynecologic surgery. The review presents an overview of the ERAS elements used and examines the effect on postoperative outcomes.

Chapter 3 describes the influence of the breakthrough series collaborative in colonic surgery on spontaneous spread to another closely related department, gynecologic surgery. Perioperative care and outcomes of gynecological procedures are compared in a retrospective multicenter study.

Chapter 4 presents a non-randomized, pre-post intervention study that examines the value of active implementation of ERAS in addition to spontaneous diffusion.

Chapter 5 describes the study protocol of a cluster randomized controlled trial comparing an innovative tailored stepwise implementation approach to the traditionally used generic breakthrough approach. The protocol provides insight into the structure of both strategies.

Chapter 6 presents and discusses the results of this SINERGY trial (stepped implementation of enhanced recovery in gynecology) on the effectiveness of implementation strategies on the adherence to the ERAS protocol and clinical outcomes, along with a process analysis to identify intervention fidelity and contextual factors that influence implementation.

Chapter 7 provides insight in the differences between the non-participating and participating hospitals of the SINERGY trial and additionally explores if and how perioperative practice changed among the non-participants.

Chapter 8 provides a general discussion of the results presented in this thesis and recommendations for future research are given.

Chapter $\mathbf{9}$ and 10 summarizes the results presented in this thesis. 


\section{REFERENCES}

1. Urbach DR, Baxter NN: Reducing variation in surgical care. BMJ 2005, 330(7505):14011402.

2. Eccles MP, Mittman BS: Welcome to Implementation Science. Implementation Science 2006, 1(1):1.

3. Grol R, Grimshaw J: From best evidence to best practice: effective implementation of change in patients' care. Lancet 2003, 362(9391):1225-1230.

4. Sackett DL, Rosenberg WM, Gray JA, Haynes RB, Richardson WS: Evidence based medicine: what it is and what it isn't. BMJ 1996, 312(7023):71-72.

5. Morris ZS, Wooding S, Grant J: The answer is 17 years, what is the question: understanding time lags in translational research. Journal of the Royal Society of Medicine 2011, 104(12):510-520.

6. Grol R: Successes and failures in the implementation of evidence-based guidelines for clinical practice. Med Care 2001, 39(8 Suppl 2):II46-54.

7. Berwick DM: Disseminating innovations in health care. JAMA 2003, 289(15):1969-1975.

8. Shekelle P, Woolf S, Grimshaw JM, Schunemann HJ, Eccles MP: Developing clinical practice guidelines: reviewing, reporting, and publishing guidelines; updating guidelines; and the emerging issues of enhancing guideline implementability and accounting for comorbid conditions in guideline development. Implement Sci 2012, 7:62.

9. Woolf SH, Grol R, Hutchinson A, Eccles M, Grimshaw J: Clinical guidelines: potential benefits, limitations, and harms of clinical guidelines. BMJ 1999, 318(7182):527-530.

10. McGlynn EA, Asch SM, Adams J, Keesey J, Hicks J, DeCristofaro A, Kerr EA: The quality of health care delivered to adults in the United States. The New England journal of medicine 2003, 348(26):2635-2645.

11. Sheldon TA, Cullum N, Dawson D, Lankshear A, Lowson K, Watt I, West P, Wright D, Wright J: What's the evidence that NICE guidance has been implemented? Results from a national evaluation using time series analysis, audit of patients' notes, and interviews. BMJ 2004, 329(7473):999.

12. Grimshaw J, Eccles M, Tetroe J: Implementing clinical guidelines: current evidence and future implications. J Contin Educ Health Prof 2004, 24 Suppl 1:S31-37.

13. Schuster MA, McGlynn EA, Brook RH: How good is the quality of health care in the United States? The Milbank quarterly 1998, 76(4):517-563, 509.

14. Fisher ES, Bynum JP, Skinner JS: Slowing the growth of health care costs-lessons from regional variation. The New England journal of medicine 2009, 360(9):849-852.

15. Cabana MD, Rand CS, Powe NR, Wu AW, Wilson MH, Abboud PA, Rubin HR: Why don't physicians follow clinical practice guidelines? A framework for improvement. JAMA 1999, 282(15):1458-1465.

16. Grimshaw JM, Eccles MP, Lavis JN, Hill SJ, Squires JE: Knowledge translation of research findings. Implement Sci 2012, 7:50.

17. Clay-Williams R, Nosrati H, Cunningham FC, Hillman K, Braithwaite J: Do large-scale hospital- and system-wide interventions improve patient outcomes: a systematic review. BMC health services research 2014, 14:369.

18. Rogers E. Diffusion of Innovations. 5th ed. New York: Free Press: 2003.

19. Cochrane Effective Practice and Organisation of Care Review Group: Data Collection Checklist. Ontario: Institute of Population Health. 2011, University of Ottawa. In. 
20. Mazza D, Bairstow P, Buchan H, Chakraborty SP, Van Hecke O, Grech C, Kunnamo I: Refining a taxonomy for guideline implementation: results of an exercise in abstract classification. Implement Sci 2013, 8:32.

21. Wensing M, van der Weijden T, Grol R: Implementing guidelines and innovations in general practice: which interventions are effective? The British journal of general practice : the journal of the Royal College of General Practitioners 1998, 48(427):991-997.

22. Grimshaw JM, Thomas RE, MacLennan G, Fraser C, Ramsay CR, Vale L, Whitty P, Eccles MP, Matowe L, Shirran L et al: Effectiveness and efficiency of guideline dissemination and implementation strategies. Health technology assessment 2004, 8(6):iii-iv, 1-72.

23. Squires JE, Sullivan K, Eccles MP, Worswick J, Grimshaw JM: Are multifaceted interventions more effective than single-component interventions in changing health-care professionals' behaviours? An overview of systematic reviews. Implement Sci 2014, 9:152.

24. Baker R, Camosso-Stefinovic J, Gillies C, Shaw EJ, Cheater F, Flottorp S, Robertson N: Tailored interventions to overcome identified barriers to change: effects on professional practice and health care outcomes. Cochrane Database Syst Rev 2010(3):CD005470.

25. Nadeem E, Olin SS, Hill LC, Hoagwood KE, Horwitz SM: Understanding the components of quality improvement collaboratives: a systematic literature review. The Milbank quarterly 2013, 91(2):354-394.

26. Kilo CM: A framework for collaborative improvement: lessons from the Institute for Healthcare Improvement's Breakthrough Series. Quality management in health care 1998, 6(4):1-13.

27. Schouten LM, Hulscher ME, van Everdingen JJ, Huijsman R, Grol RP: Evidence for the impact of quality improvement collaboratives: systematic review. BMJ 2008, 336(7659):1491-1494.

28. Kehlet H: Multimodal approach to control postoperative pathophysiology and rehabilitation. British Journal of Anaesthesia 1997, 78(5):606-617.

29. Kehlet H: Surgical stress response: does endoscopic surgery confer an advantage? World J Surg 1999, 23(8):801-807.

30. Kehlet H: Fast-track surgery-an update on physiological care principles to enhance recovery. Langenbecks Arch Surg 2011, 396(5):585-590.

31. Kehlet H, Wilmore DW: Evidence-based surgical care and the evolution of fast-track surgery. Ann Surg 2008, 248(2):189-198.

32. Lassen K, Hannemann P, Ljungqvist O, Fearon K, Dejong CH, von Meyenfeldt MF, Hausel J, Nygren J, Andersen J, Revhaug A et al: Patterns in current perioperative practice: survey of colorectal surgeons in five northern European countries. BMJ 2005, 330(7505):14201421.

33. Fearon KC, Ljungqvist O, Von Meyenfeldt M, Revhaug A, Dejong CH, Lassen K, Nygren J, Hausel J, Soop M, Andersen J et al: Enhanced recovery after surgery: a consensus review of clinical care for patients undergoing colonic resection. Clin Nutr 2005, 24(3):466-477.

34. Gustafsson UO, Hausel J, Thorell A, Ljungqvist O, Soop M, Nygren J, Enhanced Recovery After Surgery Study G: Adherence to the enhanced recovery after surgery protocol and outcomes after colorectal cancer surgery. Arch Surg 2011, 146(5):571-577.

35. Varadhan KK, Neal KR, Dejong CH, Fearon KC, Ljungqvist O, Lobo DN: The enhanced recovery after surgery (ERAS) pathway for patients undergoing major elective open 
colorectal surgery: a meta-analysis of randomized controlled trials. Clin Nutr 2010, 29(4):434-440.

36. Gustafsson UO, Scott MJ, Schwenk W, Demartines N, Roulin D, Francis N, McNaught CE, Macfie J, Liberman AS, Soop M et al: Guidelines for perioperative care in elective colonic surgery: Enhanced Recovery After Surgery (ERAS((R))) Society recommendations. World J Surg 2013, 37(2):259-284.

37. Khan S, Wilson T, Ahmed J, Owais A, MacFie J: Quality of life and patient satisfaction with enhanced recovery protocols. Colorectal Dis 2009, 12(12):1175-1182.

38. Ansari D, Gianotti L, Schroder J, Andersson R: Fast-track surgery: procedure-specific aspects and future direction. Langenbecks Arch Surg 2013, 398(1):29-37.

39. Gillissen F, Hoff C, Maessen JM, Winkens B, Teeuwen JH, von Meyenfeldt MF, Dejong CH: Structured synchronous implementation of an enhanced recovery program in elective colonic surgery in 33 hospitals in The Netherlands. World J Surg 2013, 37(5):1082-1093.

40. Gillissen F, Ament SM, Maessen JM, Dejong CH, Dirksen CD, van der Weijden T, von Meyenfeldt MF: Sustainability of an enhanced recovery after surgery program (ERAS) in colonic surgery. World J Surg 2015, 39(2):526-533.

41. Carter J: Fast-track surgery in gynaecology and gynaecologic oncology: a review of a rolling clinical audit. ISRN Surg 2012, 2012:368014.

42. Chase DM, Lopez S, Nguyen C, Pugmire GA, Monk BJ: A clinical pathway for postoperative management and early patient discharge: does it work in gynecologic oncology? Am J Obstet Gynecol 2008, 199(5):541 e541-547.

43. Gerardi MA, Santillan A, Meisner B, Zahurak ML, Diaz Montes TP, Giuntoli RL, 2nd, Bristow RE: A clinical pathway for patients undergoing primary cytoreductive surgery with rectosigmoid colectomy for advanced ovarian and primary peritoneal cancers. Gynecol Oncol 2008, 108(2):282-286.

44. Kalogera E, Bakkum-Gamez JN, Jankowski CJ, Trabuco E, Lovely JK, Dhanorker S, Grubbs PL, Weaver AL, Haas LR, Borah BJ et al: Enhanced recovery in gynecologic surgery. Obstet Gynecol 2013, 122(2 Pt 1):319-328.

45. Sidhu VS, Lancaster L, Elliott D, Brand AH: Implementation and audit of 'Fast-Track Surgery' in gynaecological oncology surgery. Aust N Z J Obstet Gynaecol 2012, 52(4):371376.

46. Ahmed J, Khan S, Lim M, Chandrasekaran TV, MacFie J: Enhanced recovery after surgery protocols - compliance and variations in practice during routine colorectal surgery. Colorectal Disease 2011, 14(9):1045-1051.

47. Schouten LM, Niessen LW, van de Pas JW, Grol RP, Hulscher ME: Cost-effectiveness of a quality improvement collaborative focusing on patients with diabetes. Med Care 2010, 48(10):884-891. 




\section{Enhanced recovery pathways in abdominal gynecologic surgery A systematic review and meta-analysis}

Jeanny J.A. de Groot

Stephanie M.C. Ament

José M.C. Maessen

Cornelis H.C. Dejong

Jos M.P. Kleijnen

Brigitte F.M. Slangen

Acta Obstet Gynecol Scand 2016; 95:382-395 


\section{ABSTRACT}

Introduction Enhanced recovery pathways have been widely accepted and implemented for different types of surgery. Their overall effect in abdominal gynecologic surgery is still underdetermined. A systematic review and meta-analysis were performed to provide an overview of current evidence and to examine their effect on postoperative outcomes in women undergoing open gynecologic surgery.

Material and methods Searches were conducted using EMBASE, MEDLINE, CINAHL, and the Cochrane Library up to June 27, 2014. Reference lists were screened to identify additional studies. Studies were included if at least four individual items of an enhanced recovery pathway were described. Outcomes included the length of hospital stay, complication rates, readmissions, and mortality. Quantitative analysis was limited to comparative studies. Effect sizes were presented as relative risks (RR) or as mean differences (MD) with $95 \%$ confidence intervals (CI).

Results Thirty-one records, involving 16 observational studies, were included. Diversity in reported elements within studies was observed. Preoperative education, early oral intake, and early mobilization were included in all pathways. Five studies, with a high risk of bias, were eligible for quantitative analysis. Enhanced recovery pathways reduced primary (MD -1.57 days, 95\%CI -2.94 to -0.20) and total (MD -3.05 days, $95 \% \mathrm{CI}-4.87$ to -1.23 ) length of hospital stay as compared to traditional perioperative care, without an increase in complication, mortality, and readmission rates.

Conclusion The available evidence based on a broad range of non-randomized studies at high risk of bias suggests that enhanced recovery pathways may reduce length of postoperative hospital stay in abdominal gynecologic surgery. 


\section{INTRODUCTION}

Despite recent technical improvements in minimally invasive surgery, open surgery still plays an essential role in gynecology [1]. Therapeutic interventions for gynecologic malignancies often require an abdominal approach. Cervical, uterine, and ovarian cancers are the three most common types of gynecologic malignancies and contribute to more than $16 \%$ of all cancers among women worldwide [2]. The introduction of the Enhanced Recovery After Surgery (ERAS) pathway, or fasttrack surgery, in colorectal surgery caused a fundamental change in perioperative management $[3,4]$. The pathway comprises a multimodal and multidisciplinary concept to optimize patient recovery after surgery following specific evidence-based recommendations $[5,6]$. Nowadays several studies have shown that the results are transferrable to other surgical disciplines [7, 8].

A number of recent systematic reviews have described the effects of ERAS, for example in colorectal surgery [9-12], esophagectomy [13, 14], and pancreatic surgery $[15,16]$. In contrast to other surgical fields, no quantitative systematic review of ERAS has been published in gynecologic surgery. Although studies have shown beneficial effects on inflammatory response and postoperative outcomes in gynecologic surgeries [17-19] and although recent reviews described current evidence on enhanced recovery pathways in gynecologic oncology [20,21], the overall effect of ERAS within open abdominal gynecologic surgery for benign and malignant conditions remains undetermined. The review of Lu et al. [20] included only randomized studies, but was unable to identify high-quality evidence. Therefore, we aimed to provide a systematic overview of the available literature on enhanced recovery pathways by including both randomized and observational studies. The review outlines which elements of enhanced recovery pathways are used in abdominal gynecologic surgery and examines the effect of those pathways on postoperative outcomes in women undergoing open abdominal surgery for malignant or benign gynecologic diseases.

\section{MATERIAL AND METHODS}

The systematic review and search strategy were prospectively registered in the international register of systematic reviews (PROSPERO 2014: CRD42014009058) [22]. With the expertise of a medical librarian, terms related to the key concepts "ERAS" and "gynecologic surgery" were combined and included in the search strategy to maximize the sensitivity of the search. Details of the literature search strategy are presented in Table S1. The electronic bibliographic databases EMBASE, MEDLINE, CINAHL, and the Cochrane Library (CENTRAL, DARE, CDSR, HTAD, and NHS EED) 
were searched from January 1, 1990 to March 19, 2014. A subsequent search was performed before final analysis in June 27, 2014. No restrictions on the type of study design and language were applied. If necessary, translations were obtained and authors were contacted to obtain full-text articles. In case of conference abstracts, related posters and full-text records were retrieved if available and authors were asked for additional information in study protocols or (un)published reports. In addition, reference lists of review articles and included studies were screened and additional studies of interest were included.

\section{Study selection}

This systematic review focused on adult women undergoing open abdominal surgery for malignant or benign gynecologic diseases. Patients receiving laparoscopic or vaginal surgery were excluded. Studies were assessed for inclusion if individual items of an enhanced recovery pathway were described and if at least one predefined primary or secondary outcome measure was reported. Studies investigating pathways that did not contain at least four enhanced recovery elements were excluded. This threshold was chosen to represent the multimodal aspect of enhanced recovery pathways and is consistent with the number used in other systematic reviews [23]. In absence of a specific consensus guideline for gynecologic surgery, the consensus guideline for rectal/pelvic surgery of the ERAS Society (www.erassociety.org) was used as framework [6]. The control group, if applicable, included conventional perioperative care. If subitems within enhanced recovery pathways were compared, the most compliant subgroup was chosen for inclusion in this review. Results of the literature searches were combined and duplicates were excluded. To identify relevant articles, titles and abstracts were screened independently by two reviewers. After this initial selection, a closer review of the eligible studies was performed using full text articles. Disagreements were resolved by consensus after consulting a third reviewer. Reasons for final exclusion were recorded. Review articles were checked for relevant references, but were excluded from the analysis.

\section{Data extraction and outcome measures}

Data extraction of included studies was performed in duplicate using data extraction forms developed after pilot testing. Discrepancies were identified and discussed to reach consensus. Data collected included the design, setting, and population of the study; type of disease and operation; details of the enhanced recovery pathway; and postoperative outcome measures. The primary outcome was the total length of postoperative hospital stay in days, defined as the primary postoperative hospital stay plus the number of days of readmission within 30 days after surgery. Length of postoperative hospital stay is often used as a surrogate marker for a successful 
enhanced recovery pathway [24]. Primary length of postoperative hospital stay in days, complication rate, readmission rate, and mortality rate were defined as secondary postoperative outcome measures. If reported, data were extracted for both the total number of complications and the number of patients with one or more complications. Additionally, time needed for functional recovery, patient satisfaction, and economic consequences were registered. Functional recovery was defined as tolerance of regular diet, adequate oral pain medication, and an independency in mobility and activities of daily living. Enhanced recovery items and outcome measures had to be explicitly stated by the study. For this review, a total of 30 enhanced recovery items were scored. The lead publication was used if more publications relating to the same study were identified and a composite data set was created. Where possible, data selection was used for studies that also included non-eligible patient or treatment groups in order to select only relevant subgroups.

\section{Risk of bias assessment}

Two reviewers independently assessed the quality of included studies using the Downs and Black checklist [25]. This instrument allows the assessment of the risk of bias of non-randomized studies. Since the number of articles dealing with ERAS in gynecologic surgery is limited, studies were eligible irrespective of the outcome of the quality assessment. The risk of bias was reported in the narrative synthesis and items were qualified as unclear when information was not reported. If only one subgroup of a study could be included, the risk of bias assessment was specifically tailored to this subgroup and not to the study as a whole. Potential publication bias was planned to be assessed by the application of funnel plots, Egger's regression test, and Begg's rank test, provided that an adequate number of trials could be included.

\section{Data synthesis and statistical analysis}

The Metaanalysis of Observation Studies in Epidemiology (MOOSE) [26] and the Preferred Reporting Items for Systematic Reviews and Metaanalyses (PRISMA) [27] were followed during the reporting process. A narrative synthesis of perioperative enhanced recovery pathways and outcomes was planned. The exact programs and outcome measures were summarized. Results of case series, describing perioperative care according to ERAS, were presented in a narrative format. Subsequent quantitative analysis using Review Manager Software (RevMan) [28] was limited to comparative studies. Dichotomous outcomes were presented as absolute numbers and related percentages. For continuous outcome variables, means and standard deviations were extracted wherever possible. If only medians and (interquartile) ranges were reported, these were presented and used for narrative analysis. For studies included in the metaanalysis, the mean and standard deviation were cal- 
culated using converting formulas presented by Hozo et al. [29]. Those estimation formulas are distribution-free and are tailored to differences in sample sizes [29]. In accordance with the Handbook of Cochrane, separately reported subgroups were combined into a single group for metaanalysis [30]. Effect sizes were presented as relative risks (RR) or as mean differences (MD) with corresponding 95\% confidence intervals (CI). Statistical heterogeneity was assessed by calculating the I-squared $\left(\mathrm{I}^{2}\right)$ statistic. A random-effects model was used for pooling when the $I^{2}$ exceeded $50 \%$, indicating the presence of heterogeneity [31]. A fixed-effects model was used when there was no evidence of heterogeneity. The studies were treated equally, regardless of the study characteristics or the reported enhanced recovery elements. Pooled analyses were performed of data from both randomized and nonrandomized studies. Subgroup analyses were carried out for benign, malignant, and mixed pathology groups separately. The chi-square test was used to test for subgroup differences. A p-value $<0.05$ was considered as statistically significant.

\section{RESULTS}

\section{Search results}

A total of 2278 records were identified by searching the selected databases (Figure 1). After excluding duplicate records and after preliminary screening of titles and abstracts, 71 full-text records were assessed for eligibility. Three full-text records were provided by the authors of the articles at request [32-34]. After systematic selection, 25 records qualified for inclusion and additional reference screening yielded another six records. Thus, a total of 31 records, involving 16 studies, met the criteria and were included in the review. Fifteen records were used for data extraction [3549], but were judged not to be the lead publication. A list of the excluded studies and reasons for exclusion is provided in Table $\mathrm{S} 2$.

\section{Study characteristics}

Subgroup selection was performed in seven studies. Three studies were randomized controlled trials in design, comparing specific elements within an enhanced recovery pathway [50-52]. Of these, two trials studied the effect of spinal anesthesia $[50,52]$ and one studied the effect of laxatives [51]. Regional anesthesia and postoperative laxatives are included in original enhanced recovery pathways [6]; therefore those study arms were judged to be more ERAS compliant and were selected for analysis. Selection was also performed in four other studies to meet the population of interest [53-56]. In those studies, only open abdominal surgery patients were selected. As such, all these studies were classified as case series in this review. So, 15 


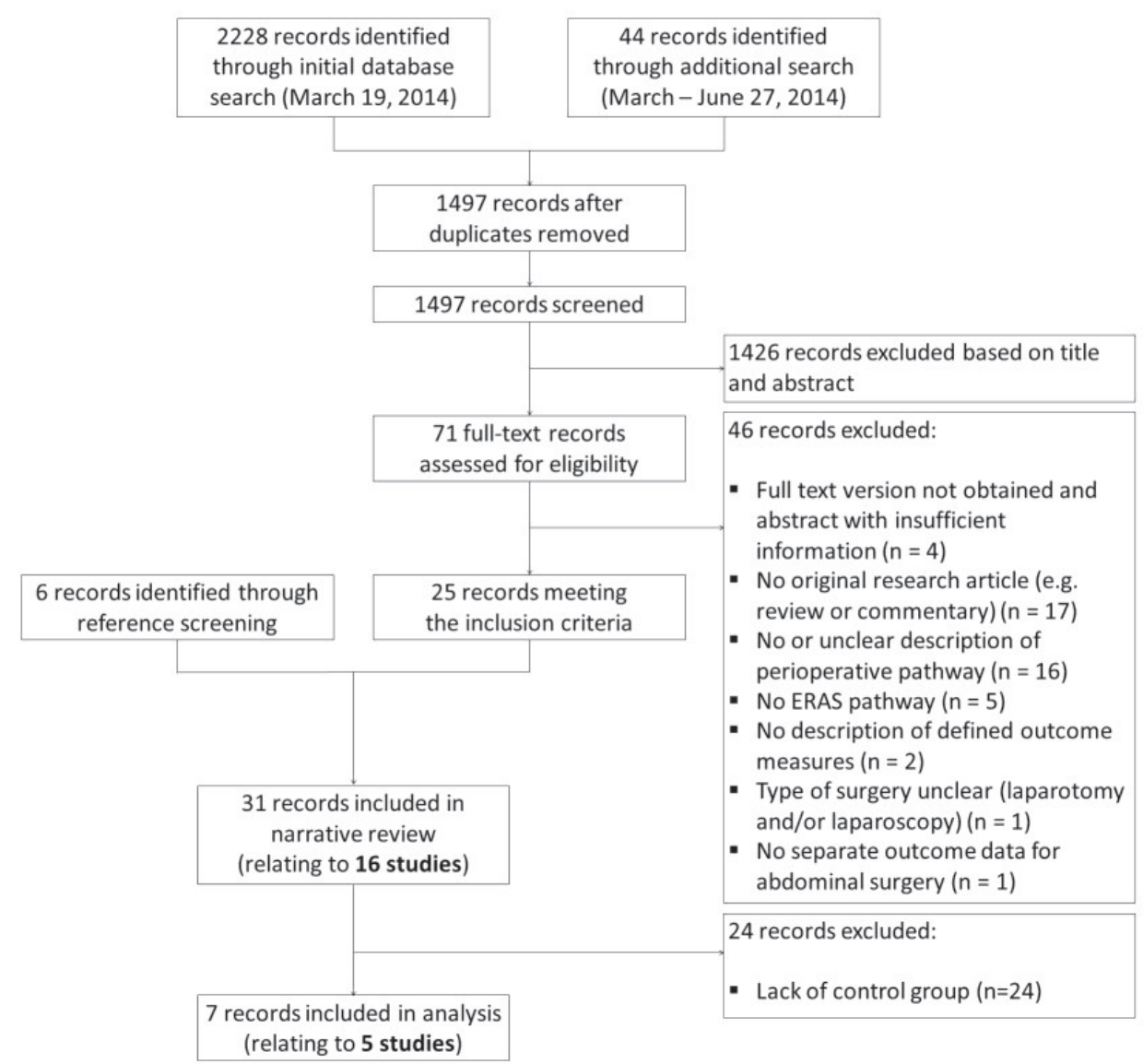

Figure 1. PRISMA diagram.

case series and one cohort study, published between 1999 and 2014, were included in the narrative review. There was one study that was published in the German language [34]; the others were written in English. The studies were conducted in Europe $(n=10)$, Australia $(n=3)$, and the United States $(n=3)$ and three of them had a multicenter design [50,55,57]. Table 1 shows the characteristics of all included studies in more detail. One study fulfilled inclusion criteria, but was reported in abstract form only [58]. Five studies compared an enhanced recovery pathway with traditional perioperative care and were used for further quantitative analysis [34, $53,57,59,60]$. One of those reported separate data for two individual subgroups (staging and cytoreductive surgeries) [53]. As stated in the method section, data of those subgroups were combined for further analysis.

A total of 1530 women were included in the intervention group receiving ERAS care. Six studies analyzed patients with benign pathology and four studies included 


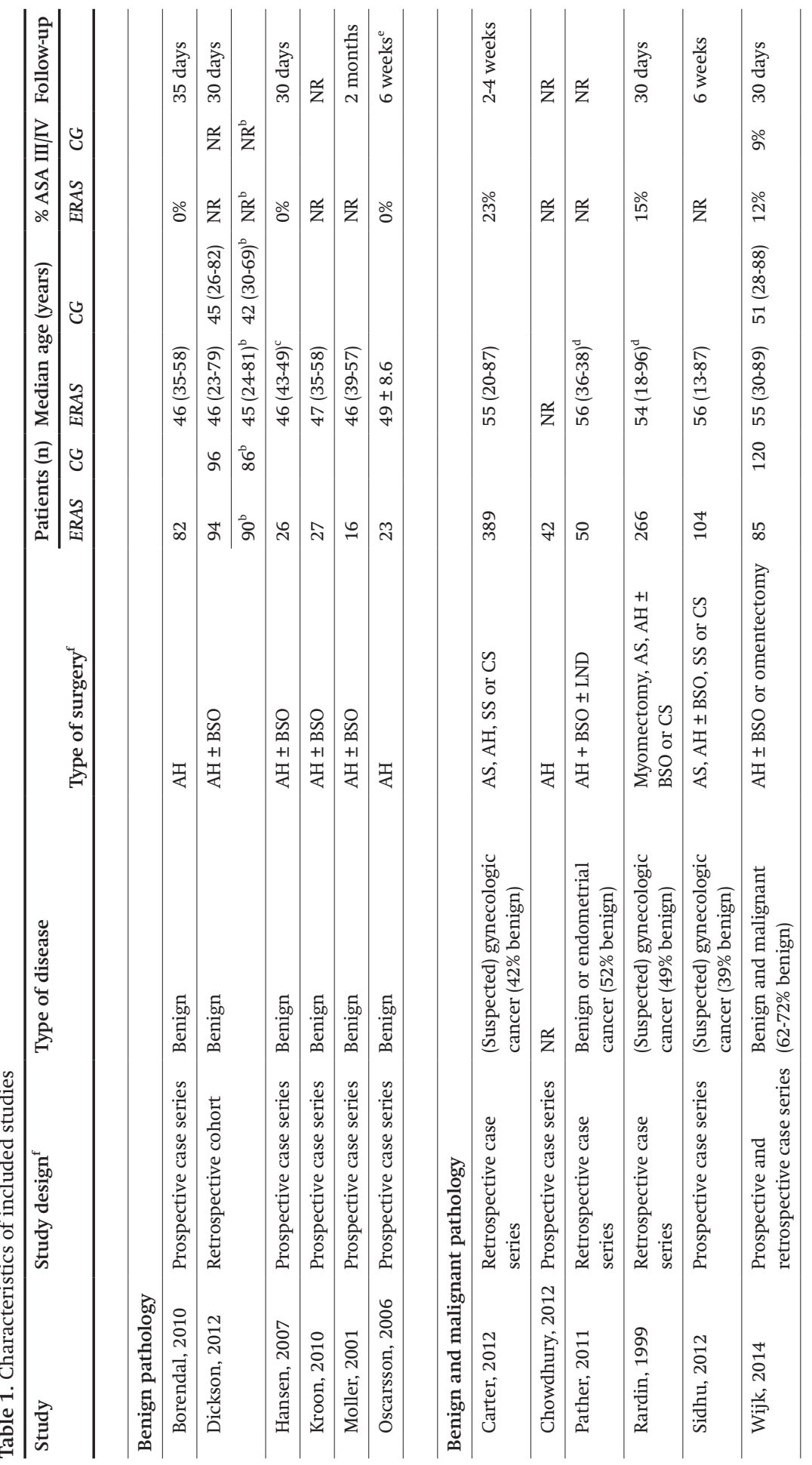




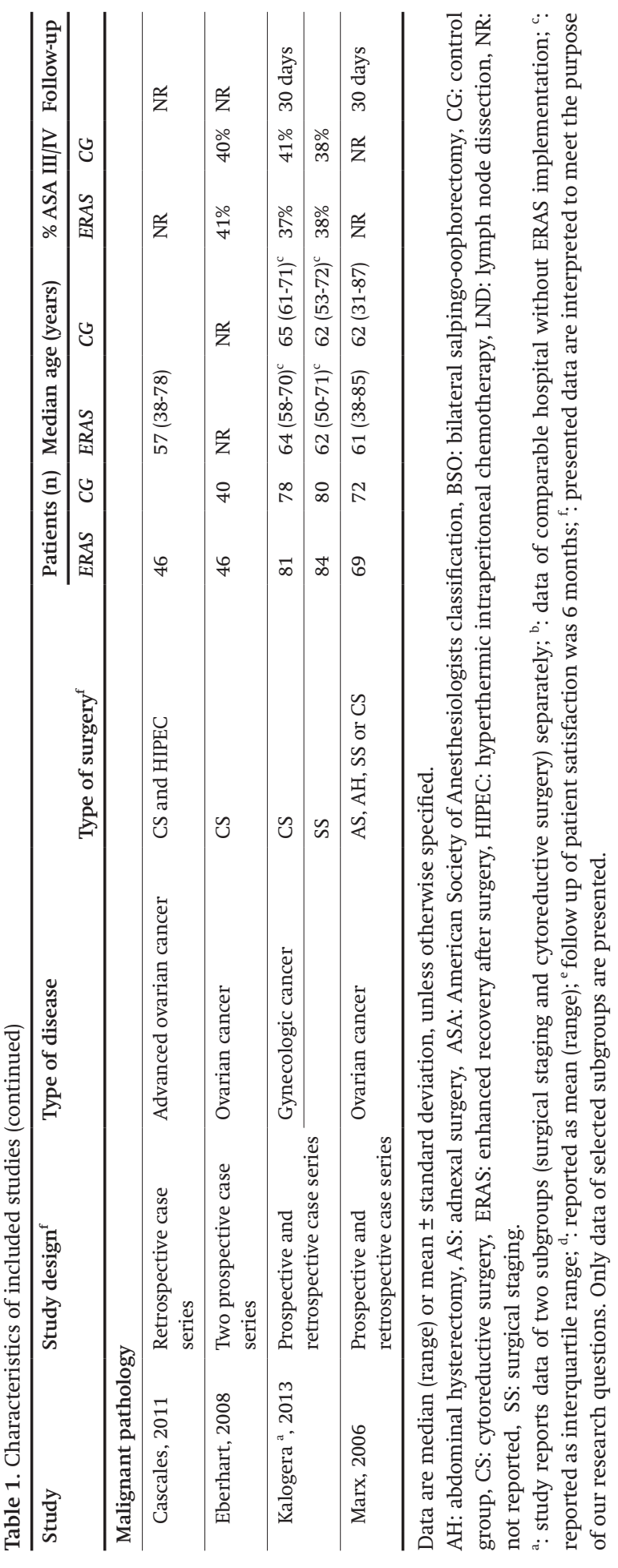


exclusively patients with malignancies (Table 1). Another four studies had a mixed group, consisting of both benign and malignant diseases at final pathology. In one study the type of disease was not specified, therefore this study was included in the mixed group as well. The type of surgery varied between those three groups and ranged from abdominal hysterectomy in the benign group to cytoreductive surgery with hyperthermic intraperitoneal chemotherapy in the malignant group. Most studies reported a follow-up of at least 30 days after surgery. The exact follow-up was not clearly stated in 4 studies [34, 52, 56, 61]. Ten out of 11 studies without a control group reported standard criteria for discharge [50-52, 54, 56, 58, 60-64]. Of the five studies with a control group, one used the same predefined discharge criteria for both groups [53], one study only used standard criteria for the intervention group [60], one study used standard criteria for the intervention group but this was not clearly described for the control group [59], one did not use standard criteria (discharge based on discretion of treating surgeon) [57], and one study did not measure length of hospital stay [34].

Due to restrictions in design and methodology, all studies were judged to have a high overall risk of bias (Table 2). We did not assign numerical composite scores, but categorized the risk of bias over the five key domains (study quality, external validity, study bias, confounding, and power of the study) to prevent diluted conclusions. Individual items of the Downs and Black checklist are presented in Table S3. Given the known limitations caused by the inadequate number of included studies, no methods were used to formally assess publication bias.

\section{Enhanced recovery pathways}

The types of pathways applied by trials are listed in Table 3. Preoperative education, early oral intake, and early mobilization were included in all pathways. Although almost all studies (15 out of 16, 94\%) applied elements of the three phases of perioperative care, considerable variation was observed in the composition of the pathways (Table 3). The number of reported enhanced recovery elements ranged between four and 21 individual items, with a median of 12.5 elements per study. Only two studies reported the level of adherence with those elements [53, 60].

\section{Outcomes}

The predefined primary and secondary outcomes are presented in Table 4. All outcomes are derived from non-randomized studies at high risk of bias. If necessary for further analysis, values were estimated from published data. The estimated values are underlined in Table 4.

Two studies, including 464 patients, reported total postoperative hospital stay within 30 days of surgery $[53,59]$. Both studies demonstrated a shorter hospital stay 
Table 2. Downs and Black risk of bias assessment

\begin{tabular}{|c|c|c|c|c|c|c|}
\hline \multirow[t]{2}{*}{ Study } & \multirow[t]{2}{*}{ Reporting } & \multirow[t]{2}{*}{ External validity } & \multicolumn{2}{|c|}{ Internal validity: } & \multirow[t]{2}{*}{ Power } & \multirow[t]{2}{*}{ Overall } \\
\hline & & & Bias & Confounding & & \\
\hline Borendal & Low & Unclear & High & High & - & High risk \\
\hline Carter & Unclear & Unclear & High & High & - & High risk \\
\hline Cascales & Unclear & Unclear & High & High & - & High risk \\
\hline Chowdhury & High & Unclear & High & High & - & High risk \\
\hline Dickson & High & Low & High & High & Low & High risk \\
\hline Eberhart & High & Unclear & High & High & High & High risk \\
\hline Hansen & Low & Low & High & High & - & High risk \\
\hline Kalogera & Unclear & Unclear & Unclear & High & Low & High risk \\
\hline Kroon & High & Unclear & High & High & - & High risk \\
\hline Marx & High & Low & Unclear & High & High & High risk \\
\hline Moller & High & Low & High & High & - & High risk \\
\hline Oscarsson & High & Unclear & High & High & - & High risk \\
\hline Pather & High & Unclear & High & High & - & High risk \\
\hline Rardin & High & Unclear & High & High & - & High risk \\
\hline Sidhu & Low & Unclear & High & High & - & High risk \\
\hline Wijk & Low & Low & Unclear & High & High & High risk \\
\hline
\end{tabular}

for patients with a malignancy in the enhanced recovery group. Fixed-effect metaanalysis of the calculated data showed a significant difference with the traditional group (mean difference (MD) -3.05 days, $95 \% \mathrm{CI}-4.87$ to -1.23 days, $p=0.001$, $\mathrm{I} 2=0 \%$ ) (Figure 2).

Consistent with the definition for this review, most studies seemed to measure primary length of hospital stay as the number of days after surgery. One study reported the number of days after admission [62] and in two studies length of stay was not defined $[56,63]$. These studies were excluded from further analysis. For one study data were converted into days [50]. The length of postoperative hospital stay following an enhanced recovery pathway ranged from 1 to 2 days among patients with a benign disease, from 2 to 4 days in the mixed population and from 5 to 7 days among patients with a malignancy (Table 4). In four studies, including 859 patients, a pre-post comparison was made between enhanced recovery pathways and traditional care $[53,57,59,60]$. In one of them, outcomes were also compared to a control hospital that did not implement an enhanced recovery pathway [57]. Primary length of postoperative hospital stay in the experimental hospital decreased, while the length at the control hospital remained unchanged. In the random-effects model, using calculated data of studies at high risk of bias, implementation of enhanced recovery pathways reduced time to discharge by 1.57 days (95\% CI -2.94 to -0.20 days, $p=0.02, I^{2}=91 \%$ ) (Figure 3 ). In subgroup analysis, the effect of enhanced recovery 


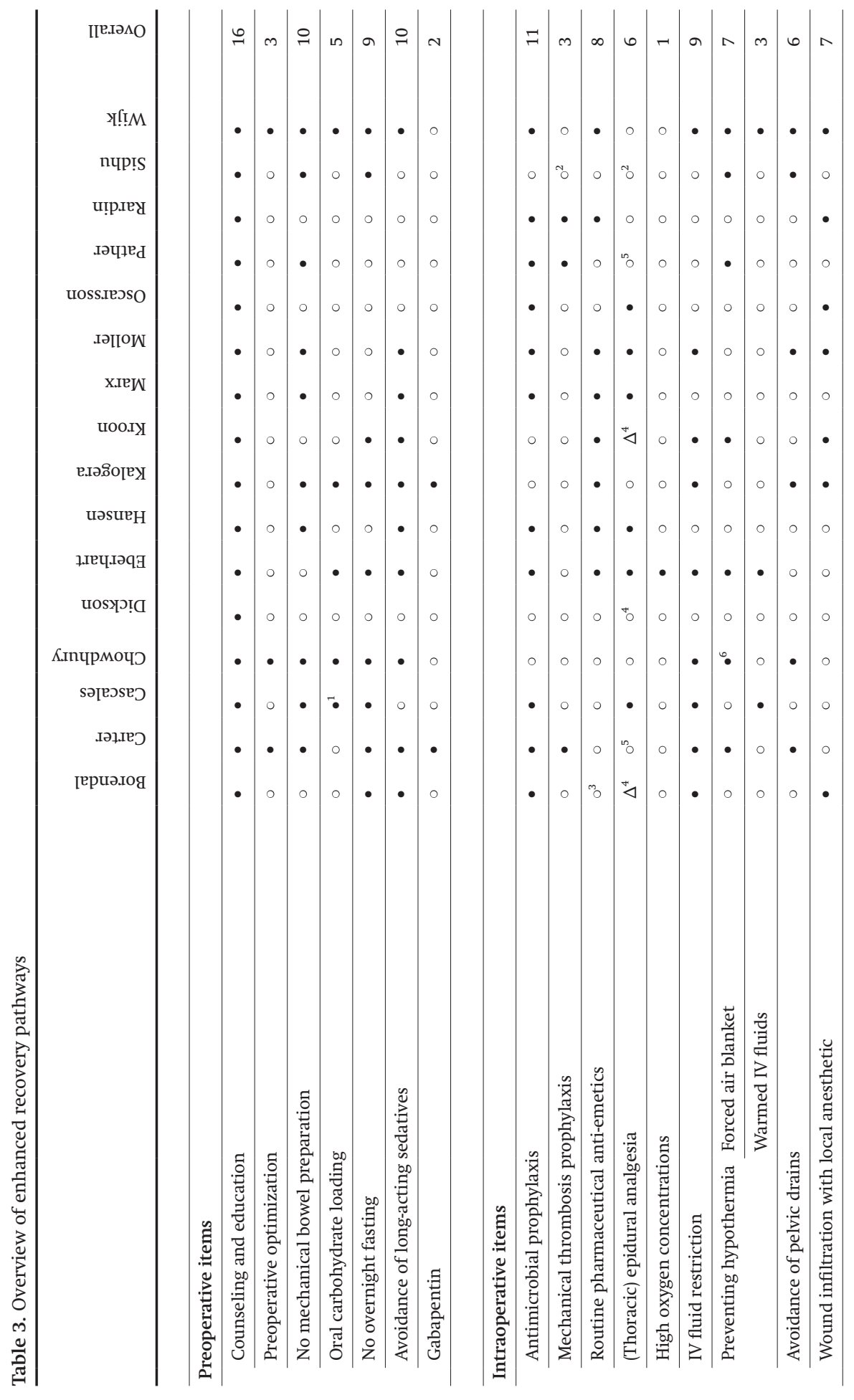




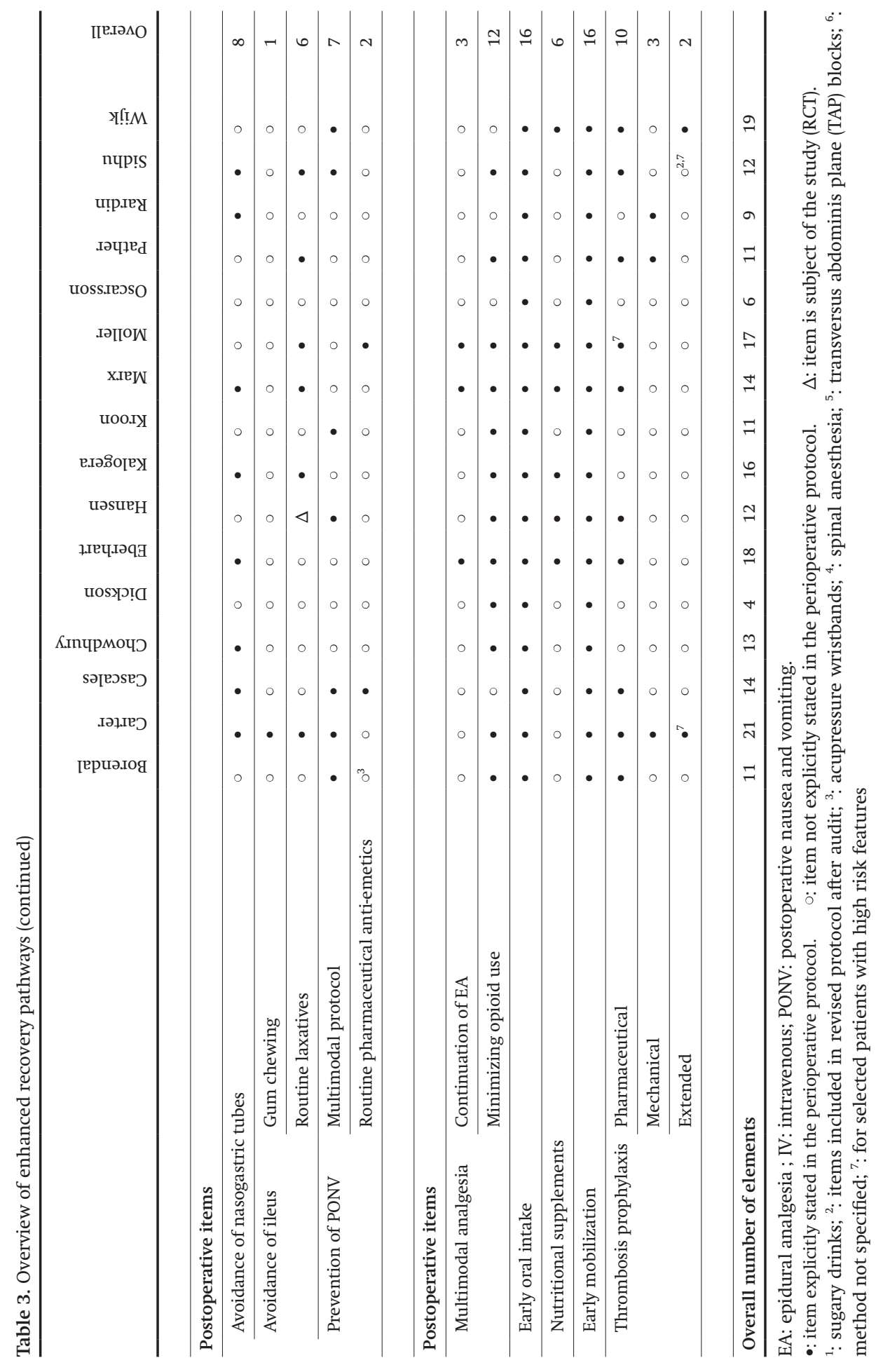




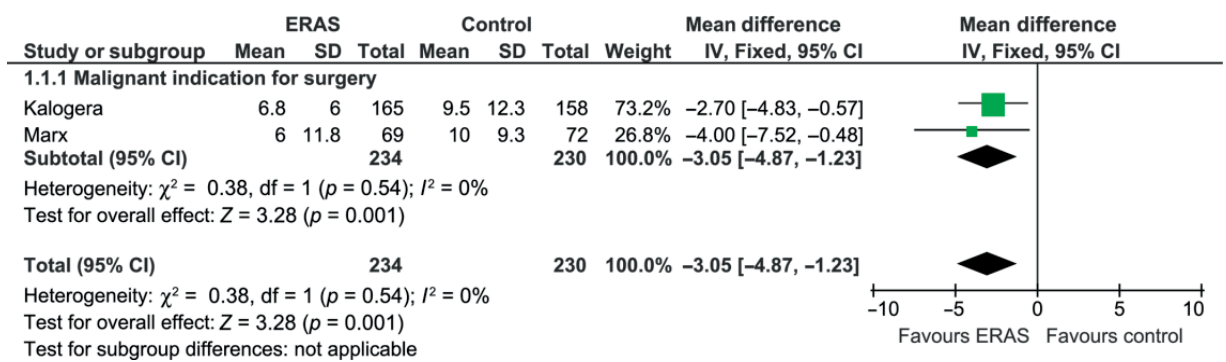

Figure 2. Meta-analyses of total length of hospital stay of enhanced recovery pathways (ERAS) versus control groups with traditional care.

pathways on primary length of postoperative hospital stay was more pronounced (test for subgroup differences $p<0.001$ ) among patients with a malignant (MD -2.42 days, $95 \%$ CI -3.61 to -1.22 days, $p<0.001, I^{2}=0 \%$ ) or benign (MD -2.0 days, $95 \%$ CI -2.65 to -1.35 days, $p<0.001$ ) indication for surgery (Figure 3). In contrast, introduction of an enhanced recovery pathway did not significantly affect the length of hospital stay in the study with a mixed (benign or malignant) patient group (MD - 0.20 days, $95 \%$ CI -0.52 to 0.12 days, $p=0.22$ ). The presented data in this study were not specified for the benign and malignant group separately.

Complication rates were analyzed separately for number of patients and total number of complications. Seven studies reported the percentage of patients with one or more complication within enhanced recovery pathways (Table 4). After a median of 35 days of follow-up, the percentage ranged from 17 to $27 \%$ (median 19\%). Two studies reported the complication rate of the control group [59, 60]. No statistically significant differences were observed between the intervention and control group (RR 0.92, 95\% CI 0.62 to $1.37, p=0.69$ ) (Figure S1). Subgroup analysis did not find a statistically significant effect either (Figure S1).

Eleven studies reported the number of complications in patients treated in an ERAS setting (Table 4), but the actual time frame was not given in two of them $[34,61]$. The total complication rate ranged from 10 to $93 \%$ (median 20\%). After 30 days of follow-up, enhanced recovery pathways showed intra- and postoperative complication rates comparable to traditional pathways (RR 1.01, 95\% CI 0.85 to 1.19, $p=0.94)$ (Figure S2) $[53,57,59,60]$. There was no heterogeneity $\left(I^{2}=0 \%, p=0.88\right)$ among the four studies included for metaanalysis and subgroup analysis revealed no statistically significant differences ( $p=0.79$ ) (Figure S2).

Readmission rates after an enhanced recovery pathway were recorded in 11 studies (Table 4). The median reported rate was $4 \%$ (range, 2 to $2 \%$ ). Four studies also examined readmission rates after traditional perioperative care $[53,57,59$, 60]. No statistically significant difference was found for enhanced recovery versus 


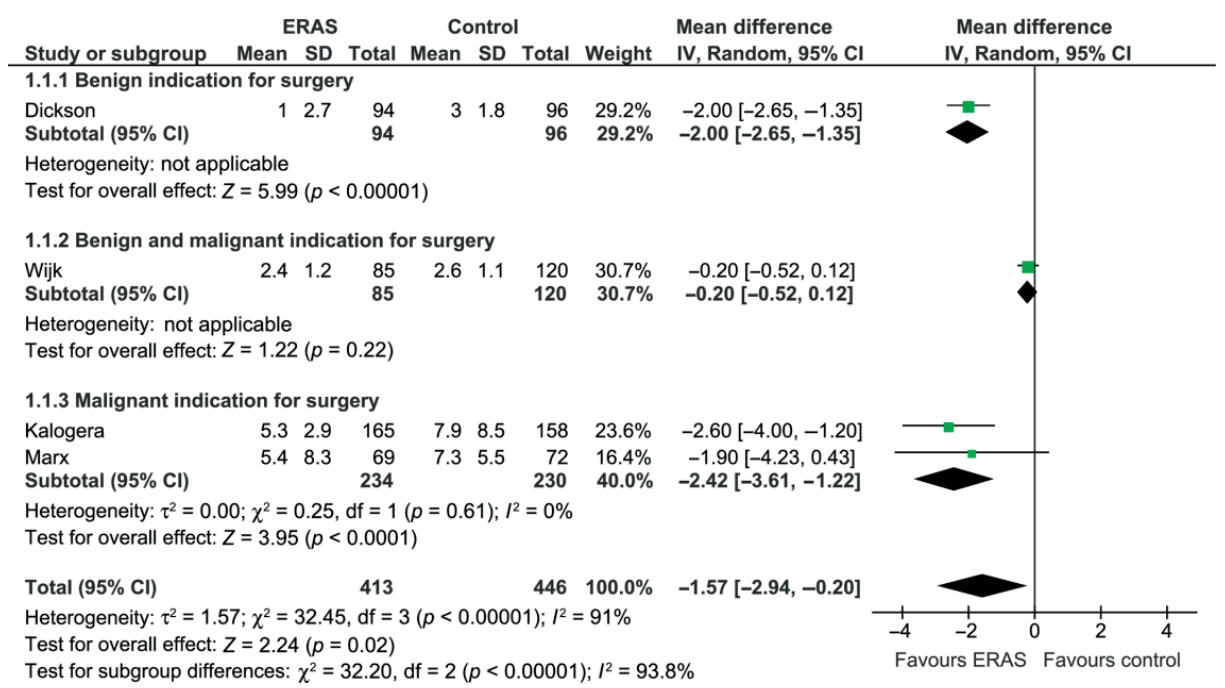

Figure 3. Meta-analyses of primary length of hospital stay of enhanced recovery pathways (ERAS) versus control groups with traditional care.

traditional pathways after 30 weeks of follow-up (RR 1.12, 95\% CI 0.74 to $1.71, p=$ $0.59)$, with no significant heterogeneity $\left(\mathrm{I}^{2}=23 \%, p=0.27\right)$ (Figure S3). No significant difference was found in any subgroup analysis (Figure S3).

Only a few studies $(n=7)$ explicitly reported mortality rates (Table 4$)$. In one study a mortality rate of $2 \%$ was observed [53]. This percentage was zero in the other studies. Based on these low event rates, quantitative analysis was not performed.

Although some of the included studies measured an individual item of functional recovery, none reported on the achievement of total functional recovery. Only two studies collected information on patient satisfaction. Kalogera et al. [53] used a self-developed questionnaire with six items (e.g. patient education and nausea management) for patients receiving ERAS-based care. A high patient satisfaction for all aspects was observed. Eberhart et al. [34] prospectively evaluated self-assessment (PPP33 questionnaire) and found an improved quality of life after the introduction of enhanced recovery pathways. One study examined costs of postoperative care before and after implementation of an enhanced recovery pathway [53]. Although not statistically significant, a tendency to a decrease in hospital costs was observed after implementation of ERAS compared with pre-implementation results. 
Table 4. Primary and secondary outcomes

\begin{tabular}{|c|c|c|c|c|c|c|c|c|c|c|c|}
\hline \multirow[t]{3}{*}{ Study } & \multirow{2}{*}{\multicolumn{2}{|c|}{$\begin{array}{l}\text { Total LOS } \\
\text { (days) }\end{array}$}} & \multirow{2}{*}{\multicolumn{2}{|c|}{$\begin{array}{l}\text { Primary LOS } \\
\text { (days) }\end{array}$}} & \multicolumn{3}{|c|}{ Complication rate (\%) } & \multirow{2}{*}{\multicolumn{2}{|c|}{$\begin{array}{l}\text { Readmission } \\
\text { rate }(\%)\end{array}$}} & \multirow{2}{*}{\multicolumn{2}{|c|}{$\begin{array}{l}\text { Mortality } \\
\text { rate (\%) }\end{array}$}} \\
\hline & & & & & $\begin{array}{l}\text { Number of } \\
\text { patients }\end{array}$ & \multicolumn{2}{|c|}{$\begin{array}{l}\text { Number of } \\
\text { complications }\end{array}$} & & & & \\
\hline & ERAS & $C G$ & ERAS & $C G$ & ERAS CG & ERAS & $C G$ & ERAS & $C G$ & ERAS & $C G$ \\
\hline \multicolumn{12}{|c|}{ Benign pathology } \\
\hline Borendal & NR & & $2(1-5)^{b}$ & & $27 \%$ & NR & & NR & & $0 \%$ & \\
\hline \multirow[t]{2}{*}{ Dickson } & NR & NR & $\underline{1 \pm 2.7}$ & $\underline{3 \pm 1.8}$ & NR & $20 \%$ & $17 \%$ & $2 \%$ & $1 \%$ & NR & NR \\
\hline & & & & & $\mathrm{NR}^{\mathrm{c}} \mathrm{NR}^{\mathrm{c}}$ & $20 \%^{\mathrm{c}}$ & $26 \%^{c}$ & $2 \%^{c}$ & $1 \%^{\mathrm{c}}$ & $\mathrm{NR}^{\mathrm{c}}$ & $\mathrm{NR}^{\mathrm{c}}$ \\
\hline Hansen & NR & & $1 \pm 0.7^{\mathrm{d}}$ & & $19 \%$ & $19 \%$ & & NR & & NR & \\
\hline Kroon & NR & & $2(1-3)$ & & NR & NR & & $4 \%$ & & NR & \\
\hline Moller & NR & & $2(2-4)$ & & $19 \%$ & $19 \%$ & & $13 \%$ & & NR & \\
\hline Oscarsson & NR & & $2.0 \pm 1.3$ & & $17 \%$ & $17 \%$ & & NR & & NR & \\
\hline
\end{tabular}

\begin{tabular}{|c|c|c|c|c|c|c|c|c|c|c|c|c|}
\hline \multicolumn{13}{|c|}{ Benign and malignant pathology } \\
\hline Carter & NR & & $3(2-27)^{e}$ & & NR & & $10 \%$ & & $4 \%$ & & NR & \\
\hline Chowdhury & NR & & $2.6(\mathrm{NR})$ & & NR & & NR & & NR & & NR & \\
\hline Pather & NR & & $3.4(2-5)^{\mathrm{e}, \mathrm{f}}$ & & NR & & NR & & $4 \%$ & & NR & \\
\hline Rardin & NR & & $2.9 \pm 2.9^{\mathrm{e}}$ & & NR & & NR & & $3 \%$ & & $0 \%$ & \\
\hline Sidhu & NR & & $4(2-18)$ & & $18 \%$ & & $21 \%$ & & $7 \%$ & & $0 \%$ & \\
\hline Wijk & NR & NR & $2.4 \pm 1.2$ & $2.6 \pm 1.1$ & $19 \%$ & $17 \%$ & $19 \%$ & $18 \%$ & $4 \%$ & $4 \%$ & NR & NR \\
\hline
\end{tabular}

\begin{tabular}{|c|c|c|c|c|c|c|c|c|c|c|c|c|}
\hline \multicolumn{13}{|c|}{ Malignant pathology } \\
\hline Cascales & NR & & $6.9 \pm 1.6$ & & NR & & $37 \%$ & & $4 \%$ & & $0 \%$ & \\
\hline Eberhart & NR & NR & NR & NR & NR & NR & $93 \%$ & $205 \%$ & NR & NR & $0 \%$ & $0 \%$ \\
\hline Kalogera $^{\text {a }}$ & $\underline{6.8 \pm 6.0}$ & $\underline{9.5 \pm 12.3}$ & $\underline{5.3 \pm 2.9}$ & $\underline{7.9 \pm 8.5}$ & NR & NR & $56 \%$ & $56 \%$ & $20 \%$ & $15 \%$ & $2 \%$ & $1 \%$ \\
\hline Marx & $6 \pm 11.8$ & $10 \pm 9.3$ & $5.4 \pm 8.25$ & $7.3 \pm \underline{\underline{5.5}}$ & $25 \%$ & $32 \%$ & $32 \%$ & $36 \%$ & $3 \%$ & $10 \%$ & $0 \%$ & $3 \%$ \\
\hline
\end{tabular}

Data are median (range) or mean \pm standard deviation, unless otherwise specified. Estimated values from published data are underlined.

CG: control group, CS: cytoreductive surgery, ERAS: enhanced recovery after surgery, LOS: length of postoperative hospital stay, NR: not reported, SD: standard deviation, SS: surgical staging

a: study reports data of two subgroups (surgical staging and cytoreductive surgery) separately and data were combined for quantitative analysis; ${ }^{\mathrm{b}}$ : in article LOS reported as hours; ${ }^{\mathrm{c}}$ : data of comparable hospital without ERAS implementation; d: reported as median \pm SD; e: excluded from further analysis, LOS not defined or measured as the number of days after admission; ${ }^{f}$ : reported as mean (range).

\section{DISCUSSION}

This review supports the theory that enhanced recovery pathways may result in a shorter length of primary and total hospital stay as compared to traditional perioperative care. A reduction of 1.57 days and 3.05 days was observed respectively, without an increase in complication, mortality, and readmission rate. The number 
of eligible studies comparing enhanced recovery to traditional care pathways in abdominal gynecologic surgery was limited and all outcomes were derived from non-randomized studies at high risk of bias. Most studies identified in this review, reported only data after the implementation of enhanced recovery pathways. Comparable outcomes were reported after implementation in studies with control groups as well as in studies without control groups. The effect on postoperative length of hospital stay was more pronounced in the malignant group. With the implementation of enhanced recovery pathways, a postoperative length of stay of 1 to 2 days can be reached for women undergoing abdominal hysterectomy for a benign indication. For women undergoing cytoreductive surgery a length of stay of 5 days might be achievable. The overall aim of enhanced recovery pathways to improve patient recovery was not structurally examined in the included studies.

Our review provides an overview of which elements are used within enhanced recovery pathways. A huge variation in the number and combination of reported enhanced recovery elements was observed. Only three elements (preoperative education, early oral intake, and early mobilization) were included in all pathways. These characteristic elements were part of the initial pathways for colorectal surgery to reduce the surgical stress response [3, 24]. Despite the inconsistency between pathways, comparable lengths of hospital stay were found within the benign, malignant, and mixed pathology subgroups. The observed variation reveals a lack of consensus on and standardization of the most important enhanced recovery elements and could have influenced overall findings. Although the ERAS Society (www. erassociety.org) published recommendations for several types of surgery and some articles describe a pathway for gynecology $[62,65,66]$, a specific consensus guideline for gynecologic surgery is still lacking. For this review, the consensus guideline for rectal/pelvic surgery of the ERAS Society was used as framework [6]. Individual items have varying levels of evidence and each item scored, may not be applicable for every gynecologic procedure or could have been added over time. This could have contributed to the observed variation in enhanced recovery pathways as well. Furthermore, due to restrictions in reporting, only specific elements could be described in the articles and conference abstracts included, instead of the full pathways that may have been applied. Although studies have shown positive effects of the individual elements, their additional effect within a fully implemented enhanced recovery pathway remains undetermined [67]. Several studies focusing on colorectal surgery have performed multivariate analysis, but results are diverse [68-70]. Besides, results are supposed to be influenced by the adherence to protocol elements [68, 69]. The limited number, heterogeneity, and the lack of actual adherence rates hinder the evaluation of the effect of individual elements. Actual adherence is an important issue; however, only two of the included studies reported their compliance rates. 
Several differences were identified regarding definitions and reporting of outcome measures and traditional care was undefined. This complexity challenges thorough evaluation of enhanced recovery pathways [23].

In the present review we analyzed the effect of enhanced recovery pathways on postoperative outcomes in gynecology. All abdominal procedures were included, in contrast to previous reviews on enhanced recovery, which included only oncologic procedures [20, 21]. Although no firm conclusions can be drawn, this review represents the largest body of information available in current literature. A wide range of surgical procedures and enhanced recovery pathways related to abdominal gynecologic surgery were included. Our approach was intentionally broad because we were aware of the limited number of studies. We have chosen to use a sensitive search strategy and to minimize exclusion criteria to ensure that important information was not missed. However, studies had to describe at least four individual elements of the pathway to be eligible for inclusion. This excluded articles that stated to perform ERAS, but did not describe their pathways. Subgroup analyses were carried out for benign, malignant, and mixed pathology groups separately to address heterogeneity in the patient populations. Isolation of the data for specific types of surgery or for different pathways was not possible due to the lack of eligible studies. Methodological quality of the studies included in this review was poor and not all studies were designed to examine the effect of enhanced recovery pathways on the predefined outcome measures. Bias and confounding could have affected outcomes and therefore the level of evidence revealed in this review must be considered to be low. The information provided is the best evidence available and we tried to deal with methodological quality by carefully reporting and discussing our findings. The use of formulas to calculate data reduced the reliability and accuracy placed on the results of the metaanalyses. To improve transparency, estimated values from published data were highlighted. Interestingly, reported results were consistent with outcomes presented in systematic reviews and metaanalyses of other surgical specialties, which supports our findings. Pooled estimates for primary length of hospital stay in systematic reviews conducted in other surgical fields showed an overall reduction of approximately 2-4 days after implementation of enhanced recovery pathways $[8-13,15,16]$. These studies reported longer primary lengths of hospital stay (range, 6-16 days) with traditional perioperative management compared to the studies included in our review (range, 3-8 days). When expressed in relative terms, the beneficial effect of enhanced recovery pathways in abdominal gynecologic surgery is comparable to the effect reported in other surgical fields.

Based on the existing evidence of ERAS pathways in other surgical specialties and based on the evidence of individual elements within abdominal surgery, executing further clinical trials comparing the effects of enhanced recovery pathways vs. strict 
traditional perioperative care in gynecology might be disputable due to ethical and methodological restrictions. Besides, with the increasing number of elements implemented in daily practice, it is questionable if control care still can be characterized as traditional. Efforts and future research should focus on tailoring pathways to gynecologic situations, on the effects of altering individual items within established pathways, and on an improved implementation of items in actual practice.

This will contribute to a higher level of perioperative care and will fulfill the actual philosophy behind enhanced recovery pathways: a reduced stress response by the structured implementation of individual and interdependent evidence-based elements [3].

In conclusion, a general overview of current evidence on enhanced recovery pathways in abdominal gynecologic surgery was provided. Only observational studies at high risk of bias were identified, showing that enhanced recovery pathways seem to reduce primary and total length of postoperative hospital stay for women undergoing open abdominal surgery for both benign and malignant indications. Although the present results are comparable with conclusions of systematic reviews conducted in other surgical fields, evidence in gynecologic surgery is weak due to the limited number and poor quality of studies as well as the diversity in reporting within these studies. 


\section{REFERENCES}

1. Gala RB, Margulies R, Steinberg A, Murphy M, Lukban J, Jeppson P, et al. Systematic review of robotic surgery in gynecology: robotic techniques compared with laparoscopy and laparotomy. J Minim Invasive Gynecol. 2014;21(3):353-61.

2. Ferlay J, Soerjomataram I, Ervik M, Dikshit R, Eser S, Mathers C, et al. GLOBOCAN 2012 v1.0, Cancer Incidence and Mortality Worldwide: IARC CancerBase No. 11 [Internet]. Lyon, France: International Agency for Research on Cancer 2013.

3. Kehlet H. Multimodal approach to control postoperative pathophysiology and rehabilitation. Br J Anaesth. 1997;78(5):606-17.

4. Fearon KC, Ljungqvist O, Von Meyenfeldt M, Revhaug A, Dejong CH, Lassen K, et al. Enhanced recovery after surgery: a consensus review of clinical care for patients undergoing colonic resection. Clin Nutr. 2005;24(3):466-77.

5. Gustafsson UO, Scott MJ, Schwenk W, Demartines N, Roulin D, Francis N, et al. Guidelines for perioperative care in elective colonic surgery: Enhanced Recovery After Surgery (ERAS®) Society recommendations. World J Surg. 2013;37(2):259-84.

6. Nygren J, Thacker J, Carli F, Fearon KC, Norderval S, Lobo DN, et al. Guidelines for perioperative care in elective rectal/pelvic surgery: Enhanced Recovery After Surgery (ERAS®) Society recommendations. World J Surg. 2013;37(2):285-305.

7. Ansari D, Gianotti L, Schroder J, Andersson R. Fast-track surgery: procedure-specific aspects and future direction. Langenbecks Arch Surg. 2013;398(1):29-37.

8. Gravante G, Elmussareh M. Enhanced recovery for non-colorectal surgery. World J Gastroenterol. 2012;18(3):205-11.

9. Spanjersberg WR, Reurings J, Keus F, van Laarhoven CJ. Fast track surgery versus conventional recovery strategies for colorectal surgery. Cochrane Database Syst Rev. 2011 (2):CD007635.

10. Varadhan KK, Neal KR, Dejong CH, Fearon KC, Ljungqvist O, Lobo DN. The enhanced recovery after surgery (ERAS) pathway for patients undergoing major elective open colorectal surgery: a meta-analysis of randomized controlled trials. Clin Nutr. 2010;29(4):434-40.

11. Zhuang CL, Ye XZ, Zhang XD, Chen BC, Yu Z. Enhanced recovery after surgery programs versus traditional care for colorectal surgery: a meta-analysis of randomized controlled trials. Dis Colon Rectum. 2013;56(5):667-78.

12. Wind J, Polle SW, Fung Kon Jin PH, Dejong CH, von Meyenfeldt MF, Ubbink DT, et al. Systematic review of enhanced recovery programmes in colonic surgery. Br J Surg. 2006;93(7):800-9.

13. Dorcaratto D, Grande L, Pera M. Enhanced Recovery in Gastrointestinal Surgery: Upper Gastrointestinal Surgery. Dig Surg. 2013;30(1):70-8.

14. Findlay JM, Gillies RS, Millo J, Sgromo B, Marshall RE, Maynard ND. Enhanced recovery for esophagectomy: a systematic review and evidence-based guidelines. Ann Surg. 2014;259(3):413-31.

15. Coolsen MM, van Dam RM, van der Wilt AA, Slim K, Lassen K, Dejong CH. Systematic review and meta-analysis of enhanced recovery after pancreatic surgery with particular emphasis on pancreaticoduodenectomies. World J Surg. 2013;37(8):1909-18.

16. Kagedan DJ, Ahmed M, Devitt KS, Wei AC. Enhanced recovery after pancreatic surgery: a systematic review of the evidence. HPB (Oxford). 2015;17(1):11-16. 
17. Antipin EE, Uvarov DN, Antipina NP, Nedashkovskii EV, Sovershaeva SL. [Effect of early multimodal rehabilitation on postoperative recovery after abdominal hysterectomy] (in Russian). Anesteziolog Reanimatol. 2013;(6):37-41.

18. Wodlin NB, Nilsson L. The development of fast-track principles in gynecological surgery. Acta Obstet Gynecol Scand. 2013;92(1):17-27.

19. Bell A, Relph S, Sivashanmugarajan V, Yoong W. Enhanced recovery programmes: Do these have a role in gynaecology? J Obstet Gynecol. 2013;33(6):539-41.

20. Lu D, Wang X, Shi G. Perioperative enhanced recovery programmes for gynaecological cancer patients. Cochrane Database Syst Rev. 2012;12:CD008239.

21. Nelson G, Kalogera E, Dowdy SC. Enhanced Recovery Pathways in Gynecologic Oncology. Gynecol Oncol. 2014;135(3):586-594.

22. de Groot J, Ament S, Slangen B, Maessen J, Kleijnen J. An overview of the experiences and achievements with enhanced recovery after surgery in abdominal gynecologic surgery: a systematic review and meta-analysis. PROSPERO 2014:CRD42014009058. Available from: http://www.crd.york.ac.uk/PROSPERO/display_record.asp?ID=CRD42014009058

23. Chambers D, Paton F, Wilson P, Eastwood A, Craig D, Fox D, et al. An overview and methodological assessment of systematic reviews and meta-analyses of enhanced recovery programmes in colorectal surgery. BMJ Open. 2014;4(5):e005014.

24. Kehlet H. Enhanced Recovery After Surgery (ERAS): good for now, but what about the future? Can J Anaesth. 2015;62(2):99-104.

25. Downs SH, Black N. The feasibility of creating a checklist for the assessment of the methodological quality both of randomised and non-randomised studies of health care interventions. J Epidemiol Community Healh. 1998;52(6):377-84.

26. Stroup DF, Berlin JA, Morton SC, Olkin I, Williamson GD, Rennie D, et al. Meta-analysis of observational studies in epidemiology: a proposal for reporting. Meta-analysis of Observational Studies in Epidemiology (MOOSE) group. JAMA. 2000;283(15):2008-12.

27. Liberati A, Altman DG, Tetzlaff J, Mulrow C, Gotzsche PC, Ioannidis JP, et al. The PRISMA statement for reporting systematic reviews and meta-analyses of studies that evaluate healthcare interventions: explanation and elaboration. BMJ. 2009;339:b2700.

28. Review Manager (RevMan) [Computer program]. Version 5.2 ed. Copenhagen: The Nordic Cochrane Centre, The Cochrane Collaboration; 2012.

29. Hozo SP, Djulbegovic B, Hozo I. Estimating the mean and variance from the median, range, and the size of a sample. BMC Med Res Methodol. 2005;5:13.

30. Higgins J, Green S. Cochrane Handbook for Systematic Reviews of Interventions Version 5.1.0 [updated March 2011]. The Cochrane Collaboration, 2011 Available from www. cochrane-handbook.org.

31. Higgins JP, Thompson SG, Deeks JJ, Altman DG. Measuring inconsistency in metaanalyses. BMJ. 2003;327(7414):557-60.

32. Antypin E, Uvarov D, Antypina N, Semenova T, Hussain A, Kirov M. Effect of enhanced recovery after surgery (ERAS) on secretion of pro-inflammatory cytokines and C-reactive protein after hysterectomy. Eur J Anaesthesiol. 2013;30:13.

33. Archer S, Staples V, Hill C, Bali A. Evaluating the experience of enhanced recovery programmes for gynaecological cancer patients. Psychooncology. 2012;21:19.

34. Eberhart LHJ, Koch T, Ploger B, Wagner U, Wulf H, Zwiorek L, et al. Beschleunigte erholung nach laparotomien bei ovarialkarzinomen - Ein vergleich zwischen traditionellem vorgehen und einem multimodalen perioperativen "fast track"-rehabilitationskonzept 
aus patientensicht. [Enhanced recovery after major gynaecological surgery for ovarian cancer - An objective and patient-based assessment of a traditional versus a multimodal "fast track" rehabilitation programme] (in German). Anasthesiol Intensivmed. 2008;49(4):180-94.

35. Borendal Wodlin N, Nilsson L, Carlsson P, Kjolhede P. Cost-effectiveness of general anesthesia vs spinal anesthesia in fast-track abdominal benign hysterectomy. Am J Obstet Gynecol. 2011;205(4):326.e1-7.

36. Kjolhede P, Borendal Wodlin N, Nilsson L, Fredrikson MM, Wijma K. Impact of stress coping capacity on recovery from abdominal hysterectomy in a fast-track programme: A prospective longitudinal study. BJOG. 2012;119(8):998-1006.

37. Kjolhede P, Langstrom P, Nilsson P, Wodlin NB, Nilsson L. The impact of quality of sleep on recovery from fast-track abdominal hysterectomy. J Clin Sleep Med. 2012;8(4):395402.

38. Nilsson L, Wodlin NB, Kjolhede P. Risk factors for postoperative complications after fast-track abdominal hysterectomy. Aust N Z J Obstet Gynaecol. 2012;52(2):113-20.

39. Wodlin NB, Nilsson L, Arestedt K, Kjolhede P, Group GS. Mode of anesthesia and postoperative symptoms following abdominal hysterectomy in a fast-track setting. Acta Obstet Gynecol Scand. 2011;90(4):369-79.

40. Wodlin NB, Nilsson L, Kjolhede P. Health-related quality of life and postoperative recovery in fast-track hysterectomy. Acta Obstet Gynecol Scand. 2011;90(4):362-8.

41. Carter J, Philp S. Development and extended experience with a fast track surgery program in a gynaecological oncology service. Open Womens Health J. 2011;5(1):22-5.

42. Carter J, Philp S. Assessing outcomes after fast track surgical management of corpus cancer. Open J Obstet Gynecol. 2011;1(3):139-43.

43. Carter J, Philp S, Arora V. Fast Track Gynaecologic Surgery in the Overweight and Obese Patient. Int J Clin Med. 2010;1(2):64-9.

44. Carter J, Philp S, Arora V. Early discharge after major gynaecological surgery: advantages of fast track surgery. Open J Obstet Gynecol. 2011;1(1):1-5.

45. Carter J, Szabo R, Sim WW, Pather S, Philp S, Nattress K, et al. Fast track surgery: a clinical audit. Aust N Z J Obstet Gynaecol. 2010;50(2):159-63.

46. Kalogera E, Bakkum-Gamez JN, Jankowski CJ, Lovely JK, Grubbs PL, Weaver AL, et al. Enhanced patient recovery in gynecologic oncology: A sea change in post-operative management. Int J Gynecol Cancer. 2012;22:E88-E9.

47. Marx CI, Rasmussen T, Jakobsen DH, Ottosen C, Lundvall L, Ottesen BS, et al. Accelereret forlob efter operation for ovariecancer. [Accelerated course after operation for ovarian cancer] (in Danish). Ugeskr Laeger. 2006 10;168(15-16):1533-6.

48. Moeller C, Kehlet H, Schouenborg L, Ottesen B, Friland S, Lund C. Fast track hysterectomy. XVI FIGO World Congress of O \& G. 2000; Abstract book 3:[46 p.]. Available from: http://onlinelibrary.wiley.com/o/cochrane/clcentral/articles/458/CN-00363458/frame. html.

49. Rhou YJ, Pather S, Loadsman JA, Campbell N, Philp S, Carter J. Direct hospital costs of total laparoscopic hysterectomy compared with fast-track open hysterectomy at a tertiary hospital: a retrospective case-controlled study. Aust N Z J Obstet Gynaecol. 2013.

50. Borendal Wodlin N, Nilsson L, Kjolhede P. The impact of mode of anaesthesia on postoperative recovery from fast-track abdominal hysterectomy: A randomised clinical trial. BJOG. 2011;118(3):299-308. 
51. Hansen CT, Sorensen M, Moller C, Ottesen B, Kehlet H. Effect of laxatives on gastrointestinal functional recovery in fast-track hysterectomy: a double-blind, placebo-controlled randomized study. Am J Obstet Gynecol. 2007;196(4):311.e1-.e7.

52. Kroon UB, Radstrom M, Hjelthe C, Dahlin C, Kroon L. Fast-track hysterectomy: a randomised, controlled study. Eur J Obstet Gynecol Reprod Biol. 2010;151(2):203-7.

53. Kalogera E, Bakkum-Gamez JN, Jankowski CJ, Trabuco E, Lovely JK, Dhanorker S, et al. Enhanced recovery in gynecologic surgery. Obstet Gynecol. 2013;122(2 Pt 1):319-28.

54. Moller C, Kehlet H, Friland SG, Schouenborg LO, Lund C, Ottesen B. Fast track hysterectomy. Eur J Obstet Gynecol Reprod Biol. 2001;98(1):18-22.

55. Oscarsson U, Sundstrom Poromaa I, Nussler E, Lofgren M. No difference in length of hospital stay between laparoscopic and abdominal supravaginal hysterectomy - A preliminary study. Acta Obstet Gynecol Scand. 2006;85(6):682-7.

56. Pather S, Loadsman JA, Mansfield C, Rao A, Arora V, Philp S, et al. Perioperative outcomes after total laparoscopic hysterectomy compared with fast-track open hysterectomy - a retrospective case-control study. Aust N Z J Obstet Gynaecol. 2011;51(5):393-6.

57. Dickson E, Argenta PA, Reichert JA. Results of introducing a rapid recovery program for total abdominal hysterectomy. Gynecol Obstet Invest. 2012;73(1):21-5.

58. Chowdhury P, Kadry M, Raslan F. Audit of the enhanced recovery programme for hysterectomy at West Middlesex University Hospital. BJOG. 2012;119:230.

59. Marx C, Rasmussen T, Hjort Jakobsen D, Ottosen C, Lundvall L, Ottesen B, et al. The effect of accelerated rehabilitation on recovery after surgery for ovarian malignancy. Acta Obstet Gynecol Scand. 2006;85(4):488-92.

60. Wijk L, Franzen K, Ljungqvist O, Nilsson K. Implementing a structured Enhanced Recovery After Surgery (ERAS) protocol reduces length of stay after abdominal hysterectomy. Acta Obstet Gynecol Scand. 2014;93(8):749-56.

61. Cascales Campos PA, Gil Martinez J, Galindo Fernandez PJ, Gil Gomez E, Martinez Frutos IM, Parrilla Paricio P. Perioperative fast track program in intraoperative hyperthermic intraperitoneal chemotherapy (HIPEC) after cytoreductive surgery in advanced ovarian cancer. Eur J Surg Oncol. 2011;37(6):543-8.

62. Carter J. Fast-track surgery in gynaecology and gynaecologic oncology: a review of a rolling clinical audit. ISRN surgery. 2012;2012:368014.

63. Rardin CR, Weisman CJ, Kim YB, Niloff JM. Early discharge after abdominal surgery: experience on a gynecologic oncology service. Gynecol Oncol. 1999;75(1):47-50.

64. Sidhu VS, Lancaster L, Elliott D, Brand AH. Implementation and audit of 'Fast-Track Surgery' in gynaecological oncology surgery. Aust N Z J Obstet Gynaecol. 2012;52(4):3716.

65. Pandeva I, Shafi MI. Perioperative care in gynaecology. Obstet Gynecol Reprod Med. 2012;22(2):38-43.

66. Torbe E, Crawford R, Acheson N. Enhanced recovery programme - improving quality. Obstet Gynecol Reprod Med. 2014;24(3):91-3.

67. Nicholson A, Lowe MC, Parker J, Lewis SR, Alderson P, Smith AF. Systematic review and meta-analysis of enhanced recovery programmes in surgical patients. Br J Surg. 2014;101(3):172-88.

68. Simpson JC, Moonesinghe SR, Grocott MPW, Kuper M, McMeeking A, Oliver CM, et al. Enhanced recovery from surgery in the UK: an audit of the enhanced recovery partnership programme 2009-2012. Br J Anaesth. 2015. 
69. Feroci F, Lenzi E, Baraghini M, Garzi A, Cantafio S, Scatizzi M. Fast-track colorectal surgery: protocol adherence influences postoperative outcomes. Int J Colorectal Dis. 2013;28(1):103-9.

70. Aarts MA, Okrainec A, Glicksman A, Pearsall E, Victor JC, McLeod RS. Adoption of enhanced recovery after surgery (ERAS) strategies for colorectal surgery at academic teaching hospitals and impact on total length of hospital stay. Surg Endosc. 2012;26(2):442-50. 


\section{SUPPLEMENTARY FILES}

Table S1. Literature search strategy

Database: Embase (OvidSP). 1974 to 2014 Week 11

\begin{tabular}{|c|c|}
\hline 1 & enhanced recovery after surgery/ \\
\hline 2 & ((enhance $\$$ or rapid or early or accelerat\$ or improv\$) adj2 (recover\$ or rehab\$)).ti,ab,ot,hw. \\
\hline 3 & (fast adj2 track\$).ti,ab,ot,hw. \\
\hline 4 & ERAS.ti,ab,ot,hw. \\
\hline 5 & (multimodal or multi modal).ti,ab,ot,hw. \\
\hline 6 & prerehab\$.ti,ab,ot,hw. \\
\hline 7 & exp clinical pathway/ \\
\hline 8 & ((critical or clinical or care or treatment) adj2 (path\$1 or pathway\$)).ti,ab,ot,hw. \\
\hline 9 & 1 or 2 or 3 or 4 or 5 or 6 or 7 or 8 \\
\hline 10 & exp gynecologic surgery/ \\
\hline 11 & hysterectom\$.ti,ab,ot,hw. \\
\hline 12 & ovariectom\$.ti,ab,ot,hw. \\
\hline 13 & oophorectom\$.ti,ab,ot,hw. \\
\hline 14 & (salpingectom\$ or salpingoophorectom\$).ti,ab,ot,hw. \\
\hline 15 & salpingostom\$.ti,ab,ot,hw. \\
\hline 16 & myomectom\$.ti,ab,ot,hw. \\
\hline 17 & 10 or 11 or 12 or 13 or 14 or 15 or 16 \\
\hline 18 & 9 and 17 \\
\hline 19 & exp gynecologic disease/ \\
\hline 20 & gynecology/ \\
\hline 21 & exp ovary/ \\
\hline 22 & exp ovary disease/ \\
\hline 23 & exp uterus/ \\
\hline 24 & exp uterus disease/ \\
\hline 25 & gyn?ecol\$.ti,ab,ot,hw. \\
\hline 26 & (ovarian or ovary or ovaries or ovarium or adnexa or fallopian).ti,ab,ot,hw. \\
\hline 27 & (cervix\$ or cervices or endocervix or ectocervix).ti,ab,ot,hw. \\
\hline 28 & $\begin{array}{l}\text { (cervical adj2 (cancer or neoplas\$ or malignan } \$ \text { or carcinoma } \$ \text { or dysplasia or patholog\$ or uterine } \\
\text { or uterus)).ti,ab,ot,hw. }\end{array}$ \\
\hline 29 & (endometr\$ or myometri\$).ti,ab,ot,hw. \\
\hline 30 & (uterine or uterus or womb\$).ti,ab,ot,hw. \\
\hline 31 & 19 or 20 or 21 or 22 or 23 or 24 or 25 or 26 or 27 or 28 or 29 or 30 \\
\hline 32 & exp surgery/ \\
\hline 33 & (surg\$ or operat\$).ti,ab,ot,hw. \\
\hline 34 & exp preoperative period/ \\
\hline 35 & perioperative period/ \\
\hline 36 & exp intraoperative period/ \\
\hline 37 & exp postoperative period/ \\
\hline
\end{tabular}




\begin{tabular}{ll}
\hline 38 & (preoperat\$ or preop or pre op).ti,ab,hw,ot. \\
\hline 39 & presurg\$.ti,ab,hw,ot. \\
\hline 40 & (preadmiss\$ or pre admiss\$ or preadmit\$ or pre admit\$).ti,ab,hw,ot. \\
\hline 41 & (perioperat\$ or intraoperat\$ or perisurg\$ or intrasurg\$).ti,ab,hw,ot. \\
\hline 42 & (postoperat\$ or postsurg\$).ti,ab,ot,hw. \\
\hline 43 & 32 or 33 or 34 or 35 or 36 or 37 or 38 or 39 or 40 or 41 or 42 \\
\hline 44 & 9 and 31 and 43 \\
\hline 45 & 18 or 44 \\
\hline 46 & exp animals/ not (exp animals/ and humans/) \\
\hline 47 & 45 not 46 \\
\hline 48 & limit 47 to yr="1990 -Current” \\
\hline
\end{tabular}


Table S2. Overview of excluded records after full-text assessment $(n=46)$

Full text version not obtained and abstract with insufficient information $(n=4)$

1. D’Amato LO, Jr., Talmage LA, Hyde K, McKnight S, Vandenbusche P. Outcomes in abdominal hysterectomy patients with benign disease. Use of physician-developed clinical protocols. J Reprod Med. 1998 Nov;43(11):975-85.

2. Guezo J. Total abdominal hysterectomy: development of a patient-centred care pathway. Nurs Stand. 2003 Oct 1-7;18(3):38-42.

3. Tucci RA, Bartels KL. Ovarian cancer surgery: a clinical pathway. Clin J Oncol Nurs. 1998 Apr;2(2):65-6.

4. Veltman R, Loppnow N. Improving care for patients having abdominal hysterectomy. Hosp Case Manag. 1999 Aug;7(8):139-42.

No original research article (e.g. review or commentary) $(n=17)$

1. Acheson N, Crawford R. The impact of mode of anaesthesia on postoperative recovery from fast-track abdominal hysterectomy: a randomised clinical trial. BJOG. 2011 Feb;118(3):271-3.

2. Ansari D, Gianotti L, Schroder J, Andersson R. Fast-track surgery: What is the procedure-specific evidence? Surg Infect. 2012 June;13 (3):A8.

3. Ansari D, Gianotti L, Schroder J, Andersson R. Fast-track surgery: procedure-specific aspects and future direction. Langenbecks Arch Surg. 2013 Jan;398(1):29-37.

4. Bell A, Relph S, Sivashanmugarajan V, Yoong W. Enhanced recovery programmes: Do these have a role in gynaecology? J Obstet Gynaecol. 2013 August;33(6):539-41.

5. Brossard P, Jaton C, Petignat P, Rehberg-Klug B. Chirurgie gynecologique et fast track. Rev Med Suisse. 201224 Oct;8(359):2011-2, 4.

6. Gerber B, Stubert J, Dieterich M, Wiessner R, Wagner K. Bedeutung der fast-track-chirurgie bei gynakologischen operationen. Gynakol Prax. 2012 First Quarter;36(1):9-17.

7. Gianpiero G, Muhammad E. Enhanced recovery for non-colorectal surgery. World J Gastroenterol. 2012;18(3):205-11.

8. Helmkamp BF, Krebs HB, Corbett SL, Trodden RM, Black PW. Radical hysterectomy: Current management guidelines. Am J Obstet Gynecol. 1997;177(2):372-4.

9. Jones K. Ambulatory gynaecology: a new concept in the delivery of healthcare for women. Gynecol Surg. 2006 2006/09/01;3(3):153-6.

10. Kelliher L, Jones C, Day A. Optimising perioperative patient care: ‘enhanced recovery’ following colorectal surgery. J Perioper Pract. 2011 Jul;21(7):239-43.

11. Lu D, Wang X, Shi G. Perioperative enhanced recovery programmes for gynaecological cancer patients. Cochrane Database Syst Rev. 2012;12:CD008239.

12. Lv D, Wang X, Shi G. Perioperative enhanced recovery programmes for gynaecological cancer patients. Cochrane Database Syst Rev. 2010 (6):CD008239.

13. Pandeva I, Shafi MI. Perioperative care in gynaecology. Obstet Gynaecol Reprod Med. 2012 February;22(2):38-43.

14. Rooth C, Sidhu A. Implementing enhanced recovery in gynaecology oncology. Br J Nurs. 2012 May 24-Jun 13;21(10):S4, S7-10, S2 passim.

15. Saleh S, Majumdar A. Enhanced recovery after surgery in obstetrics and gynaecology. BJOG. 2013 June;120:419.

16. Torbe E, Crawford R, Acheson N. Enhanced recovery programme - improving quality. Obstet Gynaecol Reprod Med. 2014 March;24(3):91-3.

17. Wodlin NB, Nilsson L. The development of fast-track principles in gynecological surgery. Acta Obstet Gynecol Scand. 2013 Jan;92(1):17-27.

No or unclear description of perioperative pathway $(n=16)$

1. Abulhassan N, Maghami S. Enhanced early recovery programme. BJOG. 2012 June;119:174.

2. Archer S, Staples V, Hill C, Bali A. Evaluating the experience of enhanced recovery programmes for gynaecological cancer patients. Psychooncology. 2012 March;21:19.

3. Doohan M, Bailey J. First experience of enhanced recovery in a tertiary gynecologic oncology centre in the UK. Gynecol Oncol. 2013 July;130 (1):e40.

4. Ghosh K, Downs LS, Padilla LA, Murray KP, Twiggs LB, Letourneau CM, et al. The implementation of critical pathways in gynecologic oncology in a managed care setting: A cost analysis. Gynecol Oncol. 2001;83(2):378-82. 
5. Haran S, Madhuri TK, Ellis P, Tailor A, Butler-Manuel S. To assess role of painfusor pumps in reducing length of stay in patients undergoing laparotomy for suspected or confirmed gynaecology malignancy. BJOG. 2014 April;121:203.

6. Krige A, Greenwood S, Bannard-Smith J. Intrathecal diamorphine analgesia for abdominal hysterectomy. Anaesthesia. 2011 September;66:56.

7. Letton C, Cheung C, Nordin A. Does an enhanced recovery integrated care pathway (ICP) encourage adherence to prescribing guidelines, accelerate postoperative recovery and reduce the length of stay for gynaecological oncology patients? J Obstet Gynaecol. 2013 Apr;33(3):296-7.

8. Mayuko H, Tomoaki Y, Nozomi O, Takahiko T, Masataka Y. Influence of anesthetic technique on postoperative oral intake in gynecological surgery. Eur J Anaesthesiol. 2013 June;30:28.

9. Morris M, Levenback C, Burke TW, Dejesus Y, Lucas KR, Gershenson DM. An outcomes management program in gynecologic oncology. Obstet Gynecol. 1997 April;89(4):485-92.

10. Narang L, Mitchelmore S, Byrne H. Cost reduction and enhanced patient experience following the introduction of enhanced recovery programme in gynaecological surgery. BJOG. 2013 June;120:439.

11. Pearson SD, Kleefield SF, Soukop JR, Cook EF, Lee TH. Critical pathways intervention to reduce length of hospital stay. Am J Med. 200115 Feb;110(3):175-80.

12. Sjetne IS, Krogstad U, Odegard S, Engh ME. Improving quality by introducing enhanced recovery after surgery in a gynaecological department: consequences for ward nursing practice. Qual Saf Health Care. 2009 Jun;18(3):236-40.

13. Smith Walker T, Hindley J, Stocker M. Introducing enhanced recovery into gynaecological surgery at a district general hospital. Gynecol Surg. 2011 September;8:S204.

14. Torbe E, Louden K. An enhanced recovery programme for women undergoing hysterectomy. Int J Gynecol Obstet. 2012 October;119:S690.

15. Ung KYC, Rosli N, Anagnostopoulos A, Macdonald RD. Early experience of enhanced recovery programme after gynaecological oncology surgery. BJOG. 2013 June;120:272.

16. Wagner L, Carlslund AM, Moller C, Ottesen B. Patient and staff (doctors and nurses) experiences of abdominal hysterectomy in accelerated recovery programme. A qualitative study. Dan Med Bull. 2004 Nov;51(4):418-21.

No ERAS pathway $(n=5)$

1. Broder MS. Early experiences with a clinical pathway for hysterectomy and myomectomy. Journal of Gynecologic Techniques. 1998;4(2):55-9.

2. Broder MS, Bovone S. Improving treatment outcomes with a clinical pathway for hysterectomy and myomectomy. J Reprod Med. 2002 Dec;47(12):999-1003.

3. Gerardi MA, Santillan A, Meisner B, Zahurak ML, Diaz Montes TP, Giuntoli IRL, et al. A clinical pathway for patients undergoing primary cytoreductive surgery with rectosigmoid colectomy for advanced ovarian and primary peritoneal cancers. Gynecol Oncol. 2008 February;108(2):282-6.

4. Jakobsen DH, Hogdall C, Kehlet H. Perioperativ behandling af ovariecancer i stadium IIIC. Ugeskr Laeger. 2010 Sep 27;172(39):2688-91.

5. Terzioglu F, Simsek S, Karaca K, Sariince N, Altunsoy P, Salman MC. Multimodal interventions (chewing gum, early oral hydration and early mobilisation) on the intestinal motility following abdominal gynaecologic surgery. J Clin Nurs. 2013 Jul;22(13-14):1917-25.

No description of defined outcome measures $(n=2)$

1. Antypin E, Uvarov D, Antypina N, Semenova T, Hussain A, Kirov M. Effect of enhanced recovery after surgery (ERAS) on secretion of pro-inflammatory cytokines and C-reactive protein after hysterectomy. Eur J Anaesthesiol. 2013 June;30:13.

2. Barr C, Madhuri TK, Butler-Manuel S, Tailor A. To assess efficacy of postoperative epidural in patients undergoing laparotomy for suspected or confirmed gynaecology malignancy. BJOG. 2013 June;120:475.

Type of surgery unclear (laparotomy and/or laparoscopy) $(n=1)$

1. Mrachek J, Dusek J, Trebesch D, Sendelbach S, Haupt J. GOALS: Bundled services to reduce the length of hospital stay in women undergoing gynecology oncology surgery. BMC Complement Altern Med. 201212 Jun;12.

No separate outcome data for abdominal surgery $(n=1)$

1. Chase DM, Lopez S, Nguyen C, Pugmire GA, Monk BJ. A clinical pathway for postoperative management and early patient discharge: does it work in gynecologic oncology? Am J Obstet Gynecol. 2008

Nov;199(5):541.e1-7 
Table S3. Risk of bias of included studies assessed by the Downs and Black checklist

\begin{tabular}{|c|c|c|c|c|c|c|c|c|c|c|c|c|c|c|c|c|}
\hline & 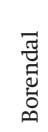 & 悫 & 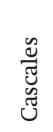 & 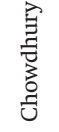 & 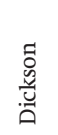 & $\begin{array}{l}\text { 䔍 } \\
\text { 莺 } \\
\text { 至 }\end{array}$ & 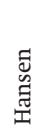 & $\begin{array}{l}\widetilde{\Xi} \\
0 \\
0 \\
\tilde{0}\end{array}$ & $\begin{array}{l}\text { ठี } \\
\text { 齐 }\end{array}$ & 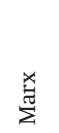 & $\frac{\vec{\Xi}}{\overrightarrow{0}}$ & $\begin{array}{l}\text { : } \\
\text { W } \\
\text { t్ల } \\
0\end{array}$ & 节 & 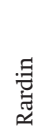 & 壳 & $\stackrel{3}{3}$ \\
\hline Q1: Aim & Yes & Yes & Yes & Yes & Yes & Yes & Yes & Yes & Yes & Yes & Yes & Yes & Yes & Yes & Yes & Yes \\
\hline Q2: Main outcomes & Yes & Yes & Yes & Yes & Yes & Yes & Yes & Yes & Yes & Yes & Yes & Yes & Yes & Yes & Yes & Yes \\
\hline Q3: Patients characteristics & Yes & Yes & Yes & Yes & Yes & Yes & Yes & Yes & Yes & Yes & Yes & Yes & Yes & Yes & Yes & Yes \\
\hline Q4: Interventions & Yes & Yes & Yes & Yes & Yes & Yes & Yes & Yes & Yes & Yes & Yes & Yes & Yes & Yes & Yes & Yes \\
\hline Q5: Principal confounders & Yes & Yes & Yes & No & Yes & Yes & Yes & Yes & No & Yes & Yes & Yes & Yes & Yes & Yes & Yes \\
\hline Q6: Main findings & Yes & Yes & Yes & No & Yes & Yes & Yes & Yes & No & Yes & Yes & Yes & Yes & Yes & Yes & Yes \\
\hline Q7: Random variability & Yes & Yes & Yes & No & Yes & Yes & Yes & Yes & Yes & Yes & Yes & Yes & Yes & No & Yes & Yes \\
\hline Q8: Adverse events & Yes & Yes & Yes & No & Yes & Yes & Yes & Yes & No & Yes & Yes & Yes & No & No & Yes & Yes \\
\hline Q9: Lost to follow up & Yes & UTD & UTD & No & UTD & Yes & Yes & UTD & No & UTD & Yes & Yes & UTD & UTD & Yes & Yes \\
\hline Q10: Actual p-values & Yes & NA & NA & NA & No & No & Yes & Yes & No & No & No & No & Yes & Yes & Yes & Yes \\
\hline Q11:Subjects representative (source) & Yes & Yes & Yes & Yes & Yes & Yes & Yes & Yes & Yes & Yes & Yes & Yes & Yes & Yes & Yes & Yes \\
\hline Q12: Subjects representative (prop.) & Yes & Yes & Yes & UTD & Yes & Yes & Yes & Yes & UTD & Yes & Yes & Yes & UTD & Yes & Yes & Yes \\
\hline Q13: Representative facilities & UTD & UTD & UTD & UTD & Yes & UTD & Yes & UTD & UTD & Yes & Yes & UTD & UTD & UTD & UTD & Yes \\
\hline Q14: Blinding of subjects & No & No & No & No & UTD & No & No & UTD & No & UTD & No & No & No & No & No & UTD \\
\hline Q15: Blinding of assessors & UTD & UTD & UTD & UTD & Yes & No & Yes & UTD & UTD & UTD & UTD & UTD & UTD & UTD & UTD & UTD \\
\hline Q16: Data dredging & Yes & No & No & Yes & No & Yes & Yes & Yes & Yes & Yes & Yes & Yes & No & No & Yes & Yes \\
\hline Q17: Adjusted analyses & NA & NA & NA & NA & UTD & UTD & NA & Yes & NA & Yes & NA & NA & NA & NA & NA & Yes \\
\hline Q18: Statistics & Yes & NA & NA & NA & Yes & No & Yes & Yes & Yes & Yes & Yes & Yes & Yes & Yes & Yes & Yes \\
\hline Q19: Compliance & UTD & UTD & UTD & UTD & UTD & UTD & UTD & Yes & UTD & UTD & UTD & UTD & UTD & UTD & UTD & Yes \\
\hline Q20: Outcome measures & Yes & Yes & Yes & No & Yes & Yes & Yes & Yes & Yes & Yes & Yes & Yes & Yes & Yes & Yes & Yes \\
\hline Q21: Same population & NA & NA & NA & NA & Yes & Yes & NA & Yes & NA & Yes & NA & NA & NA & NA & NA & Yes \\
\hline Q22: Recruitment over time & NA & NA & NA & NA & Yes & Yes & NA & Yes & NA & Yes & NA & NA & NA & NA & NA & Yes \\
\hline Q23: Randomization & No & No & No & No & No & No & No & No & No & No & No & No & No & No & No & No \\
\hline Q24: Allocation concealment & No & No & No & No & No & No & No & No & No & No & No & No & No & No & No & No \\
\hline Q25: Adjustment for confounding & NA & No & No & No & No & Yes & NA & Yes & NA & Yes & NA & NA & NA & Yes & Yes & No \\
\hline Q26: Loss of follow-up & Yes & UTD & UTD & UTD & UTD & UTD & Yes & UTD & UTD & UTD & UTD & Yes & UTD & Yes & Yes & UTD \\
\hline Q27: Power calculation & NA & NA & NA & NA & Yes & No & NA & Yes & NA & No & NA & NA & NA & NA & NA & No \\
\hline
\end{tabular}

NA: not appropriate, UTD: unable to determine 


\begin{tabular}{|c|c|c|c|c|c|c|c|c|}
\hline Study or Subgroup & \multicolumn{2}{|c|}{ Experimental } & \multicolumn{2}{|c|}{ Control } & \multicolumn{2}{|r|}{ Risk Ratio } & \multicolumn{2}{|c|}{$\begin{array}{c}\text { Risk Ratio } \\
\text { M-H, Fixed, } 95 \% \mathrm{Cl} \\
\end{array}$} \\
\hline \multicolumn{7}{|c|}{ 1.1.1 Benign and malignant indication for surgery } & & \\
\hline $\begin{array}{l}\text { Wijk } \\
\text { Subtotal }(95 \% \mathrm{Cl})\end{array}$ & 16 & $\begin{array}{l}85 \\
85\end{array}$ & 20 & $\begin{array}{l}120 \\
120\end{array}$ & $\begin{array}{l}42.4 \% \\
42.4 \%\end{array}$ & $\begin{array}{l}1.13[0.62,2.05] \\
\mathbf{1 . 1 3}[0.62,2.05]\end{array}$ & & \\
\hline $\begin{array}{l}\text { Total events } \\
\text { Heterogeneity: Not ap } \\
\text { Test for overall effect: }\end{array}$ & $\begin{array}{c}16 \\
\text { plicable } \\
Z=0.40(\end{array}$ & $=0.69$ & 20 & & & & & \\
\hline \multicolumn{9}{|c|}{ 1.1.2 Malignant indication for surgery } \\
\hline $\begin{array}{l}\text { Marx } \\
\text { Subtotal }(95 \% \mathrm{Cl})\end{array}$ & 17 & $\begin{array}{l}69 \\
69\end{array}$ & 23 & $\begin{array}{l}72 \\
72\end{array}$ & $\begin{array}{l}57.6 \% \\
57.6 \%\end{array}$ & $\begin{array}{l}0.77[0.45,1.31] \\
0.77[0.45,1.31]\end{array}$ & & \\
\hline \multicolumn{9}{|c|}{$\begin{array}{l}\text { Heterogeneity: Not applicable } \\
\text { Test for overall effect: } Z=0.96(P=0.34)\end{array}$} \\
\hline Total $(95 \% \mathrm{Cl})$ & & 154 & & 192 & $100.0 \%$ & $0.92[0.62,1.37]$ & & \\
\hline Total events & 33 & & 43 & & & & & \\
\hline $\begin{array}{l}\text { Heterogeneity: } \mathrm{Chi}^{2}= \\
\text { Test for overall effect: } \\
\text { Test for subqroup diff }\end{array}$ & $\begin{array}{l}0.88, d f= \\
Z=0.40( \\
\text { erences: }\end{array}$ & $\begin{array}{l}P=0 \\
=0.69 \\
I^{2}=0 .\end{array}$ & $\begin{array}{l}35) ; l^{2}=0 \\
\text { 37. } d f=1\end{array}$ & & 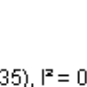 & & $\begin{array}{ll}0.5 & 0.7 \\
\text { Favours ERAS }\end{array}$ & $\begin{array}{cc}1.5 & 2 \\
\text { Favours control }\end{array}$ \\
\hline
\end{tabular}

Figure S1. Meta-analyses of complication rate (number of patients) of enhanced recovery pathways (ERAS) versus control groups with traditional care.

ERAS Control Risk Ratio Risk Ratio Study or Subgroup Events Total Events Total Weight M-H, Fixed, 95\% Cl M-H, Fixed, $95 \% \mathrm{Cl}$

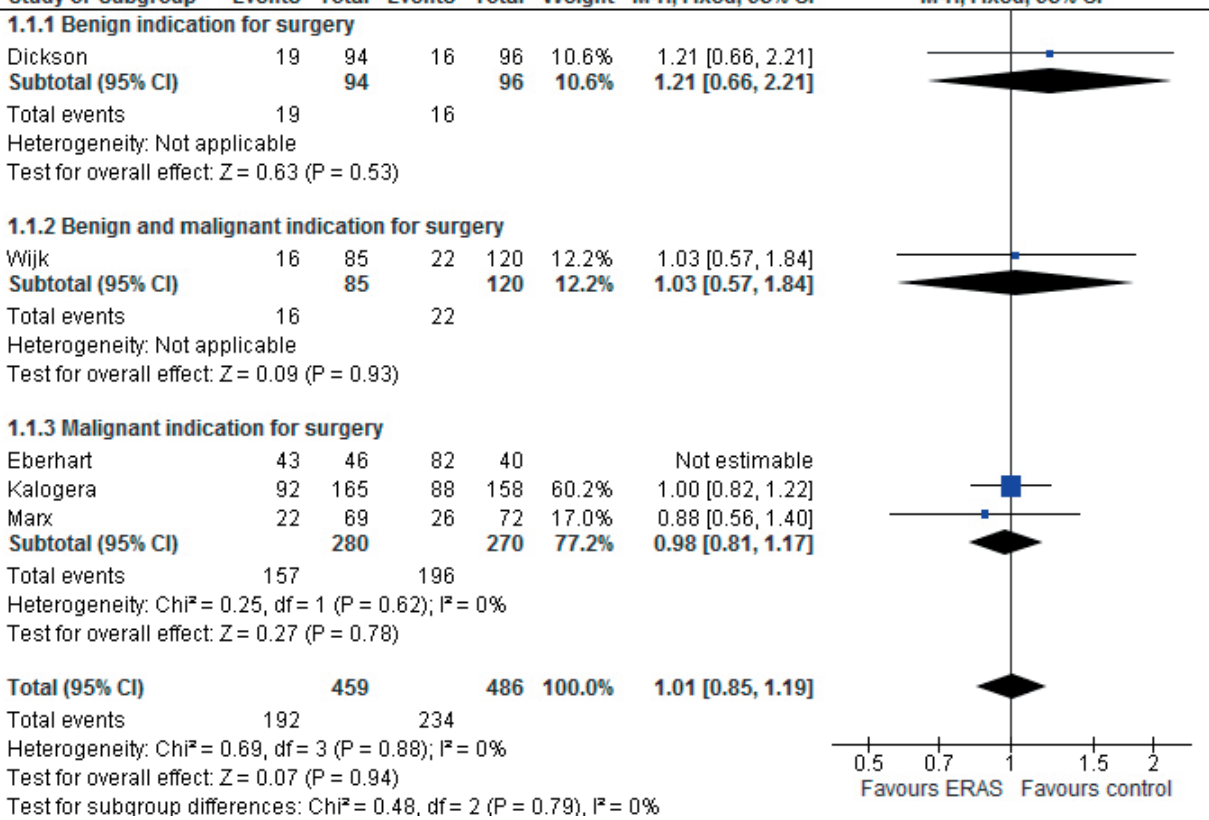

Figure S2. Meta-analyses of complication rate (total number of complications) of enhanced recovery pathways (ERAS) versus control groups with traditional care. 


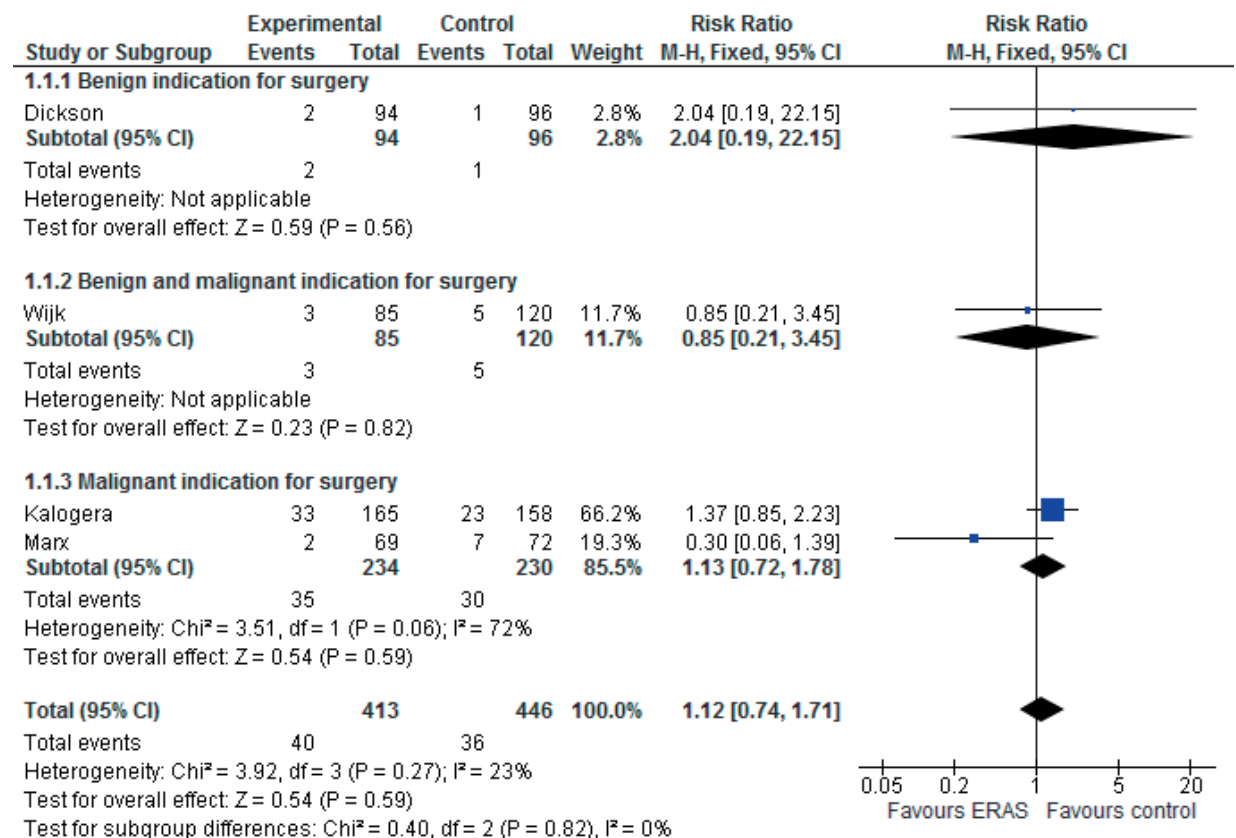

Figure 6. Meta-analyses of readmission rate of enhanced recovery pathways (ERAS) versus control groups with traditional care. 



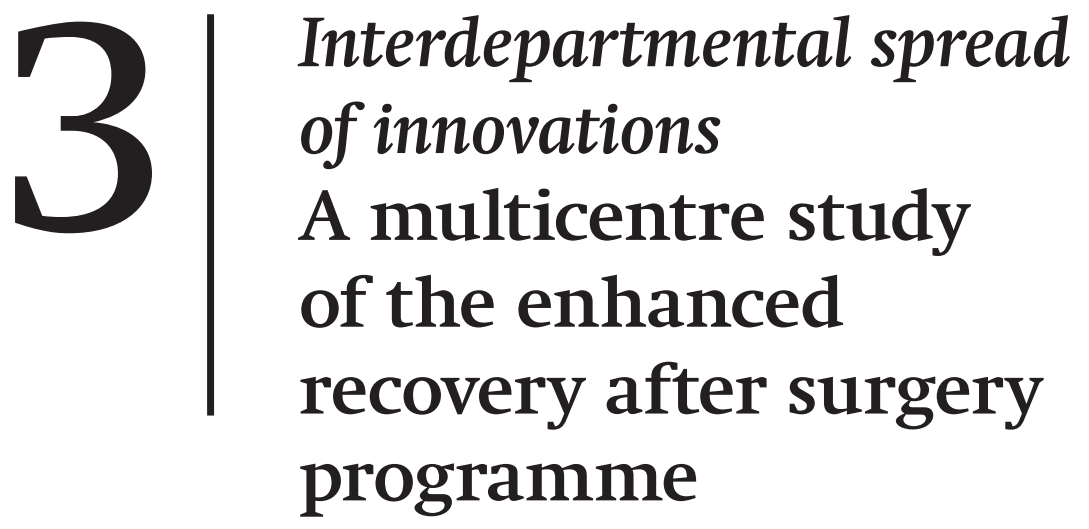

Jeanny J.A. de Groot

José M.C. Maessen

Cornelis H.C. Dejong

Bjorn Winkens

Roy F.P.M. Kruitwagen

Brigitte F.M. Slangen

Trudy van der Weijden

on behalf of all the members of the study group

World J Surg 2018; 42:2348-2355 


\section{ABSTRACT}

Background Spread of evidence-based innovations beyond pioneering settings is essential to improve quality of care. This study aimed to evaluate the influence of a national project to implement 'Enhanced Recovery After Surgery' (ERAS) among colorectal teams on the spread of this innovation to gynaecological procedures.

Methods A retrospective observational multicentre study was performed of a consecutive sample of patients who underwent major elective gynaecological surgery in 2012-2013. Ten Dutch hospitals (294 patients) had participated in a colorectal breakthrough project implementing ERAS on a nationwide basis and were assigned to the intervention group. Thirteen hospitals (390 patients) that had not participated in this project acted as controls. Outcome measures were time to functional recovery and total length of postoperative hospital stay. Multilevel models adjusted for clustering and baseline demographics were used for analysis. The uptake of ten selected perioperative care elements was evaluated for each hospital.

Results The estimated mean difference (95\% confidence interval) between the intervention and control hospitals was -0.3 (-0.9 to 0.3$)$ days in the time to recovery and 0.2 (-0.8 to 1.3) days in the total length of hospital stay. The mean ( \pm standard deviation) absolute rate of implemented perioperative care elements per hospital was $28.9 \pm 14.9 \%$ in the control, versus $29.3 \pm 11.1 \%$ in the intervention group $(p=0.934)$.

Conclusion The results suggest that the benefits of implementation of an evidencebased innovation such as the ERAS protocol are restricted to the participating teams, and do not automatically spread successfully to other surgical teams in the same hospital. 


\section{INTRODUCTION}

Innovations in healthcare are essential to ensure progress and to achieve the highest quality of care [1]. Despite the potential impact on outcomes, change of practice is generally slow and challenging [2]. Quality improvement (QI) programmes are widely used to achieve change by applying a systematic approach. A growing body of evidence on their effectiveness has become available in recent years [3]. QI programmes, such as the collaborative breakthrough method $[4,5]$, provide support to spread innovations between stakeholders during the improvement process [6]. Spread of health innovations beyond this pioneering setting is necessary to continue improving quality of care. Studies have recognised that spread is also important to sustain innovation effects after an implementation project has ended [7]. Nevertheless, improvements often remain adopted by a restricted group of innovators [8].

Greenhalgh et al. [9] described a continuum between unplanned (diffusion) and planned (dissemination) mechanisms to achieve spread. Following this broad concept, we defined spread as the process through which effective innovations are adopted from one setting to another. Spread of clinical knowledge between departments can be hampered by intra-organisational barriers, such as group membership $[10,11]$. A multiple case-study demonstrated that hospitals are able to spread both processes and content to other medical-surgical units using deliberate and active approaches to change (dissemination) [12]. Limited knowledge exists about the full continuum of local spread mechanisms when dissemination is not promoted and supported at a higher, national level.

We hypothesised that the experiences and tools gained after running a QI project among colorectal surgical teams would stimulate spread of a universal perioperative innovation to another closely related intra-organisational department, such as the gynaecological department. To examine this influence, we compared perioperative care and outcomes of gynaecological procedures in hospitals in which the colorectal surgical teams had taken part in a QI project [13], with the procedures performed by gynaecologists in hospitals in which colorectal surgical teams had not participated in the QI project.

\section{MATERIAL AND METHODS}

\section{Design}

A 1-year breakthrough project was used to implement 'Enhanced Recovery After Surgery' (ERAS) across 33 colorectal surgery departments in the Netherlands between 2006 and 2009 [13]. A significant change in practice was achieved, resulting 
in reduced length of hospital stay [13]. The spread from colorectal to gynaecological surgery was selected for analysis in the current retrospective multicentre study. The gynaecology and colorectal surgical teams were closely related and specialists cooperated regularly during surgical procedures, but departments operated independently. This observational study was exempt from ethics review (Medical Ethical Committee of the Maastricht University, METC13-5-031 and METC14-5-083).

\section{Inclusion criteria}

Dutch hospitals authorised to carry out major gynaecological cancer surgery were invited to participate. In the Netherlands, hospitals are required to meet a minimum number of 20 major ovarian cancer surgical procedures per year for authorisation. Both university and non-university teaching hospitals have the capacity to fulfil this criterion. The hospitals that had participated in the colorectal breakthrough project were included in the intervention group. Authorised hospitals that had not participated in an international or national project implementing ERAS for colorectal surgery acted as controls. One hospital participated in an international collaboration to develop and implement ERAS [14] and took the lead as an expert centre in the national implementation of ERAS in colorectal surgery [13]. As such, this hospital did not fulfil the criteria for the intervention or the control group and was excluded. The spread of ERAS towards gynaecology within this pioneering centre and the effects after active implementation have been described elsewhere [15]. The hospitals that regularly refer patients to an extended care facility for inpatient rehabilitation after surgery were excluded to prevent biased outcome measures. Hospitals with a combined ward for gynaecological and colorectal surgery could participate, but were registered as such.

A consecutive sample of patients who underwent elective open surgery for gynaecological malignancies were audited. Eligible patients were aged at least 18 years and had a suspected or proven diagnosis of ovarian, uterine, or cervical cancer. Surgical procedures included open exploratory, staging, and cytoreductive procedures in 2012 and 2013.

\section{Description of the innovation}

The uptake of the multifaceted ERAS guideline was evaluated. Based on the best available evidence, ERAS consists of several perioperative recommendations to reduce time of recovery after surgery [16]. Although originally developed for colorectal surgery, the programme is not exclusive to this specialty [17]. To date, more surgical specialties, such as gastric [18] and pancreatic surgery [19], urology [20], and gynaecology [21, 22], have adopted ERAS and have tailored guidelines to specific procedures or to local policies [23]. Individual elements of ERAS, such as the avoidance of drains, have been shown to be effective in gynaecological surgery for several years [24-28]. 


\section{Outcome measures}

Primary outcomes were the time to functional recovery after surgery (FR) and the total length of postoperative hospital stay (TLOS) in days. Recovery was reached when patients tolerated oral food and oral analgesia and regained their mobility. TLOS included the number of nights a patient stayed in the hospital after surgery plus, if appropriate, the length of readmission. The secondary outcome included the degree of local implementation of single ERAS elements; for each hospital, this was dichotomised using a target of $70 \%$ adherence as a minimum degree of implementation [29, 30]. A combined score per element was calculated. Mortality and readmission rates were registered.

\section{Data collection}

Retrospective review of medical records was performed up to 30 days postoperatively. In considering the Dutch volume norm, a maximum of 30 patients per hospital were audited. Hospital types were categorised as university and non-university teaching hospitals. There is one specialised cancer centre, which was categorised as a university hospital. The type of gynaecological cancer, histological subtype, and type of incision were specified. To obtain accurate information, data of ten ERAS elements were registered (Table 1). These elements covered the three perioperative phases of care, and their combination represents an ERAS management.

Table 1 ERAS elements included in retrospective analysis

\begin{tabular}{ll}
\hline Preoperative phase & Omission of mechanical bowel preparation \\
\cline { 2 - 2 } Operative phase & Use of carbohydrate load \\
\hline Postoperative phase & Avoidance of drains \\
\cline { 2 - 2 } & Early ambulation on the day of surgery (sitting on edge of bed) \\
\hline Scheduled postoperative administration of laxatives \\
\hline Eral feeding on POD 1 \\
\hline Early ambulation on POD 1 (sitting on chair three times a day) \\
\hline Discontinuation of intravenous fluids on POD 2 \\
\hline Discontinuation of urinary catheter on POD 2 \\
\hline
\end{tabular}

ERAS, enhanced recovery after surgery; POD, postoperative day

\section{Data analysis}

A descriptive analysis of baseline characteristics was performed. For categorical variables, differences were analysed using the chi-square test or Fisher's exact test. The independent-samples t-test was used for numerical variables. To account for clustering of patients within hospitals, linear multilevel methods were used to evaluate differences in time to FR and TLOS. The models incorporated hospital 
identification as a random effect, whereas study group, hospital type, presence of a combined ward, age ( $<60$ versus $\geq 60$ ), American Society of Anaesthesiologists classification, type of cancer, histological subtype, and type of incision were included as fixed effects. Missing outcome values were not imputed, since likelihood-based methods were used. The estimated marginal means (EMM) and mean differences (MD), corresponding 95\% confidence intervals (CI), and intra-class correlation coefficients (ICC) were presented. Unadjusted differences in the median time to FR and TLOS were analysed at hospital level using the Mann Whitney $U$ test because of the relatively small number of hospitals (13 versus 10). A reduction in time to FR and TLOS of more than one day was assumed to be clinically relevant. A value of $p \leq$ 0.005 was considered statistically significant. Analyses were performed with IBM SPSS Statistics for Windows, version 21.0 (Armonk, NY, USA).

\section{RESULTS}

A total of 684 medical records from 23 hospitals were audited (Fig. 1). Ten hospitals had followed the breakthrough strategy for colorectal surgery and were allocated to the intervention arm. The other hospitals were regarded as controls ( $n=13$ ). There were no statistically significant between-group differences in demographic data regarding the hospitals (Table 2). Some differences in the indication for surgery were noted between patient groups.

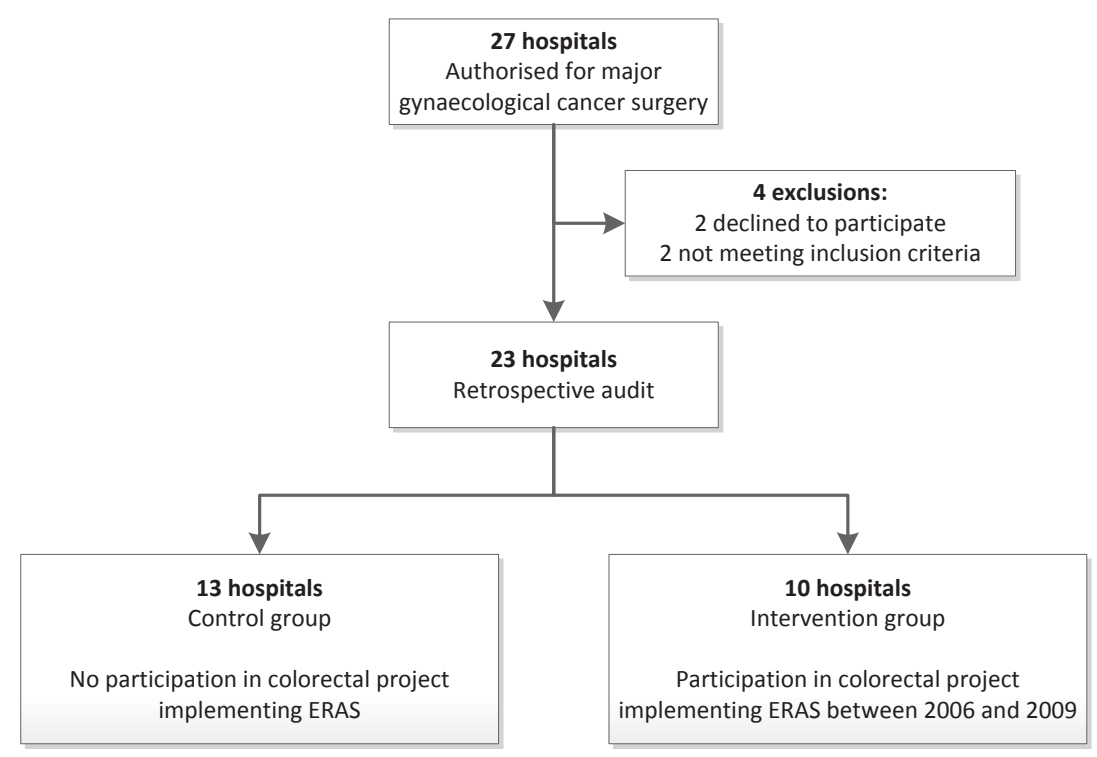

Figure 1. Study flow chart 
Table 2 Baseline demographics of hospitals and patients according to observational group

\begin{tabular}{|c|c|c|c|}
\hline & Control & Intervention & $p$ value ${ }^{\mathrm{b}}$ \\
\hline \multicolumn{4}{|l|}{ Hospital demographics } \\
\hline Number of hospitals & 13 & 10 & \\
\hline Number of beds per hospital ${ }^{a}$ & $642.3 \pm 304.5$ & $812.1 \pm 189.8$ & $0.138^{c}$ \\
\hline Type of hospital & & & $0.660^{d}$ \\
\hline University medical centre & $4(30.8)$ & $2(20.0)$ & \\
\hline Non university teaching hospital & $9(69.2)$ & $8(80.0)$ & \\
\hline \multicolumn{4}{|l|}{ Hospital ward } \\
\hline Combined with colorectal surgery & $1(7.7)$ & $2(20.0)$ & $0.560^{d}$ \\
\hline \multicolumn{4}{|l|}{ Patient demographics } \\
\hline Number of patients & 390 & 294 & \\
\hline Age, years ${ }^{a}$ & $61.4 \pm 12.0$ & $62.6 \pm 12.4$ & $0.212^{c}$ \\
\hline Age, years & & & 0.016 \\
\hline$<60$ & $164(42.1)$ & $97(33.0)$ & \\
\hline$\geq 60$ & $226(57.9)$ & $197(67.0)$ & \\
\hline ASA classification & & & 0.092 \\
\hline Class I/II & $352(90.3)$ & $253(86.1)$ & \\
\hline Class III/IV & $38(9.7)$ & $41(13.9)$ & \\
\hline Gynaecological cancer type & & & 0.045 \\
\hline Ovarian & $289(74.1)$ & $231(78.6)$ & \\
\hline Uterine & $72(18.5)$ & $54(18.4)$ & \\
\hline Cervical & $29(7.4)$ & $9(3.1)$ & \\
\hline Histological subtype & & & 0.981 \\
\hline Benign & $46(11.8)$ & $36(12.2)$ & \\
\hline Borderline / hyperplasia & $30(7.7)$ & $22(7.5)$ & \\
\hline Malignant & $314(80.5)$ & $236(80.3)$ & \\
\hline Type of incision & & & 0.842 \\
\hline Midline & 377 (96.7) & 285 (96.9) & \\
\hline Transverse & $13(3.3)$ & $9(3.1)$ & \\
\hline
\end{tabular}

Values in parentheses are percentages unless indicated otherwise;

ASA, American Society of Anaesthesiologists

${ }^{\mathrm{a}}$ values are mean \pm standard deviation.. ${ }^{\mathrm{b}}$ Chi-square test, except ${ }^{\mathrm{c}}$ independent samples t-test and ${ }^{\mathrm{d}}$ Fisher's exact test.

FR was registered for $97.2 \%$ of the patients in the control and for $98.6 \%$ in the intervention group. Data on the time to FR were missing in five patients in the control and in one patient in the intervention group. In addition, two patients in the control group were transferred to another hospital before recovery was reached. These patients were lost to follow up. Mortality rate was comparable between the 
control $(\mathrm{n}=4,1.0 \%)$ and intervention $(\mathrm{n}=3,1.0 \%)$ groups $(p>0.999)$. The hospitals' median time to FR was 4.0 days (between-hospital range 3.0-6.0) in the control group, and 4.0 days (between-hospital range 3.0-5.0) in the intervention group ( $p=0.218$ ). Following adjustment for baseline variables, multilevel modelling showed an MD of -0.3 days (95\% CI -0.9 to 0.3 ) in FR between patients treated in intervention hospitals compared to treatment in a control hospital. The EMM was 4.2 days (95\% CI 3.4-5.0) in the control group compared to 3.9 days (95\% CI 3.1-4.8) in the intervention group $(p=0.319)$ (Fig. 2). The ICC for FR was 0.03 .

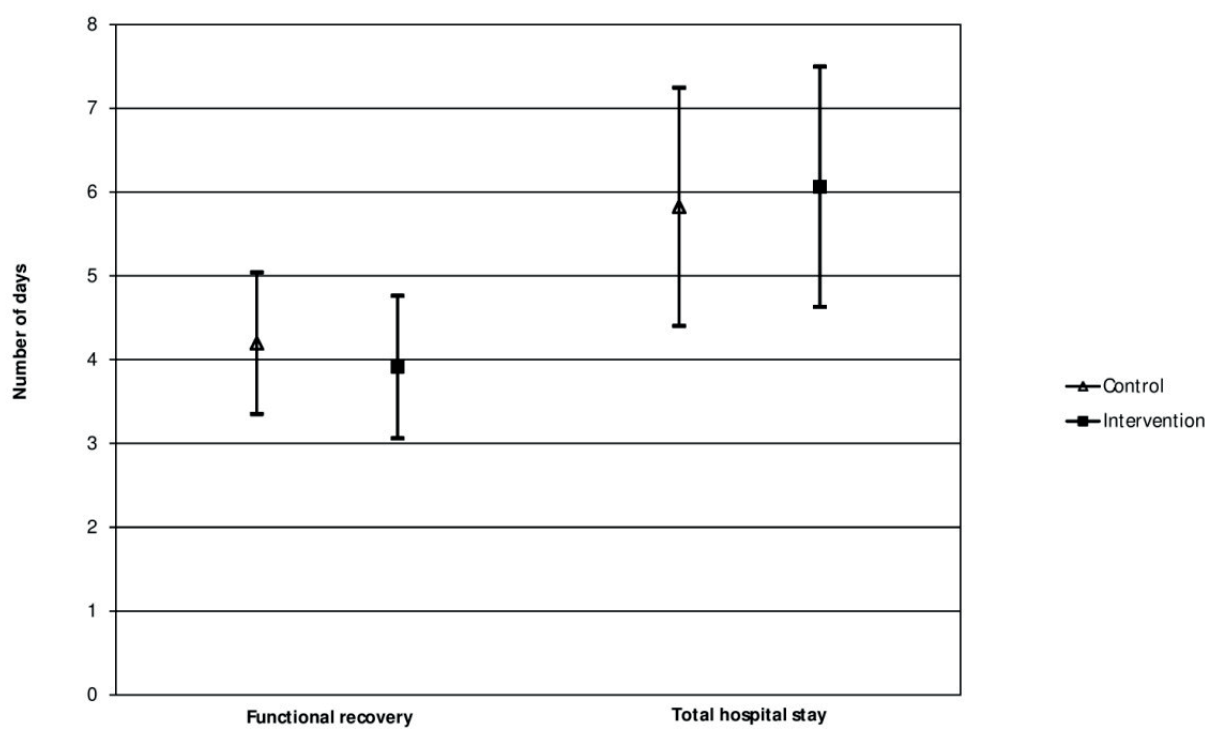

Figure 2. Model estimated marginal means in days

The median TLOS was 6.0 days in the control hospitals (between-hospital range 4.0-7.0) and 5.5 days for hospitals in the intervention group (between-hospital range 3.5-10.5) ( $p=0.777)$. The observed effect remained statistically nonsignificant after adjusting for covariates (MD 0.2 days; $95 \%$ CI -0.8 to 1.3). The EMM was 5.8 days (95\% CI 4.4-7.2) in the control group compared to 6.1 days (95\% CI 4.6-7.5) in the intervention group (Fig. 2). The ICC was 0.06 . In total, 12 patients (3.1\%) from the control and 14 patients $(4.8 \%)$ in the intervention group had to be readmitted ( $p=0.254$ ).

The degree of implementation within hospitals was based on the adherence to ten selected ERAS elements. Omission of mechanical bowel preparation, the use of local analgesia, and the avoidance of abdominal drains were the most common elements adhered to in both study groups (Fig. 3). Adoption of postoperative ERAS elements was low. No statistically significant differences were found between groups. In the control group, three hospitals (23\%) implemented at least half the elements in daily 
practice compared to one hospital (10\%) in the intervention group ( $p=0.604)$. None of these were hospitals with a combined ward for colorectal and gynaecological surgery patients. The mean absolute rate of implemented elements per hospital was $28.9 \%$ (SD 14.9\%) in the control and 29.3\% (SD 11.1\%) in the intervention group ( $p=$ 0.934).

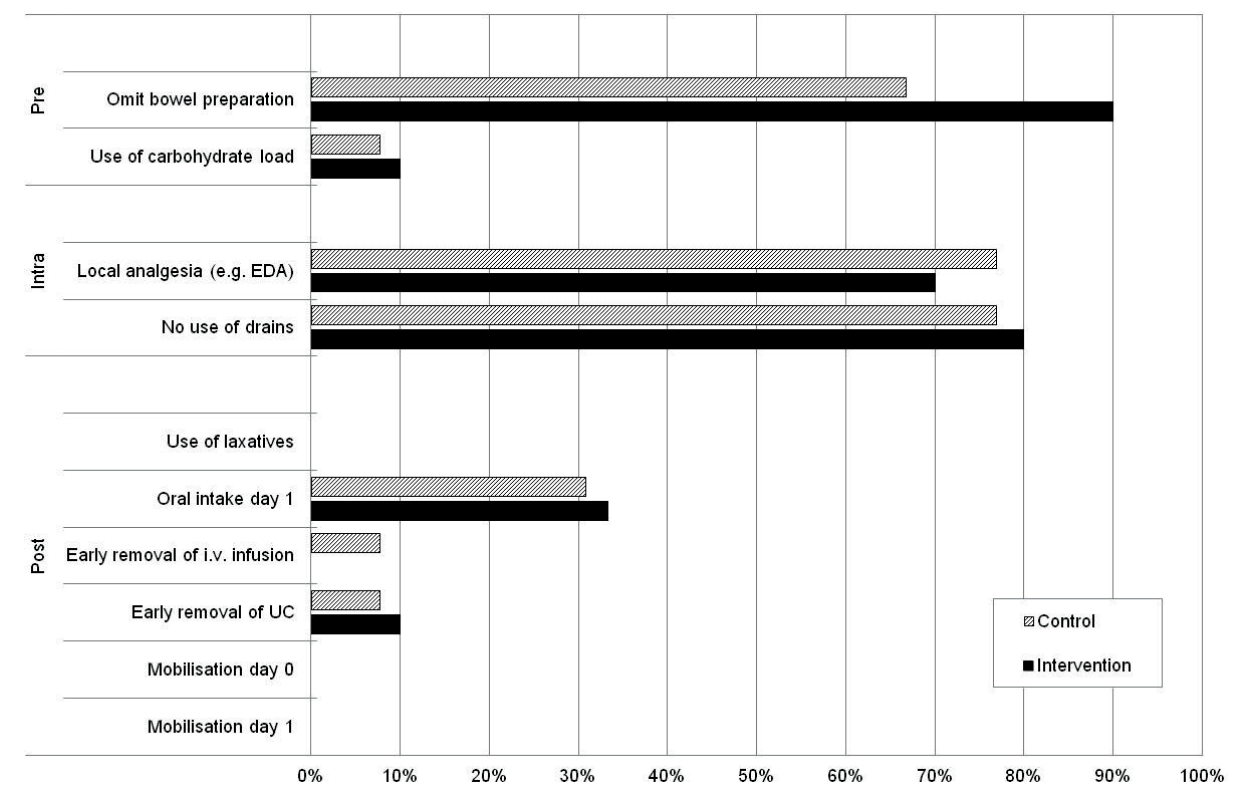

Figure 3. Percentage of implemented (> 70\%) enhanced recovery elements among hospitals

\section{DISCUSSION}

Despite the acquired knowledge and positive experiences gained from the implementation of ERAS in colorectal surgery, this study showed that almost no spread took place in the closely related department of gynaecology in the 5 years following implementation. Multilevel regression analysis, taking clustering of hospitals and baseline demographics into account, demonstrated no differences in outcomes between gynaecological procedures performed in hospitals that had participated in the colorectal QI project, and the hospitals that did not. Even the hospitals with a mixed ward for colorectal and gynaecological surgery did not adopt the majority of ERAS elements.

None of the single ERAS elements was regularly adopted by all the hospitals, despite the clear evidence already available for many years [21, 22]. This finding confirms the outcomes of a single centre study in an early-adopter hospital [15] 
and emphasises the challenge to spread and actively implement ERAS in actual practice [31]. Greenhalgh et al. [9] have described several characteristics of innovations that are required to facilitate spread. Although the multifaceted and behaviour changing aspects of ERAS may render implementation in actual practice difficult, the innovation is particularly suitable to spread within organisations. ERAS principles are universal and effectiveness has been demonstrated [32-34]. Despite the complex multifaceted structure, implementation can be achieved by using small, manageable steps. Benefits are almost directly visible for both the innovators and patients. Furthermore, the need for a multidisciplinary approach should facilitate intra-organisational spread.

This study included hospitals that participated in the original breakthrough strategy [13]. The breakthrough strategy is designed to spread innovations across multiple organisations over a short period of time [4]. Participants learned to apply practical skills to improve care and all participating hospitals reduced length of hospital stay and/or had high adherence rates [13]. Therefore, the expertise acquired should give them the capacity to guide local spread. In this study, we tried to analyse the full continuum of local spread without promotion and guidance at a higher, national level. None of the hospitals achieved a complete spread of ERAS and just one hospital in the intervention group implemented at least half of the ERAS elements. Unfortunately, we lack consistent information about the sustainability of ERAS in colorectal surgery in those hospitals.

Labgaa et al. [35] recently noted that structured implementation of ERAS in one speciality (colorectal surgery) induced a transition of elements towards another speciality (liver surgery) within the same department in patients treated by the same team. Evidence is limited about the effect of conducting a systematic QI programme on the spread of innovations between departments. It has been shown frequently that changing behaviour within healthcare organisations is challenging and complex $[2,3,36]$. Although results need to be interpreted with caution, we believe this study emphasises those findings and shows that barriers are even higher than generally expected. Awareness about the barriers might be the first step in breaking through apparent boundaries and creating a culture of change. During recent years, the shift from a traditional function-oriented towards a process-oriented structure of hospitals has gained attention [37]. The functional grouping of departments in the Netherlands seems to hamper the multidisciplinary efforts to optimise patient care. Breaking through this group membership facilitates knowledge sharing between different groups of healthcare providers. Furthermore, the involvement of intra-organisational knowledge brokers may promote spread of innovations across health-related sectors [38]. A recent review described knowledge brokers as intermediaries to exchange knowledge across the varying stakeholders and settings, 
but demonstrated that the availability of evidence about their effectiveness within healthcare is limited so far [39]. In line with other studies, we found a gap in discharge $[40,41]$. Unnecessary prolonged hospitalisation might be partially reduced by applying structured discharge planning, or by using an integrated discharge team [42].

The multicentre design strengthens conclusions and provides more generalisability of results. To our knowledge, the national comparison of interdepartmental spread between hospitals that had previously followed a QI programme, and those that had not, is unique. A limitation might be that the data abstractor was not blinded. We do not have any sign however that this has led to data manipulation, given the independent position. Otherwise, the fact that the data were extracted by one and the same person can be looked upon as a strength, as it guaranteed a standardised approach for the audit. Retrospective review of medical records has methodological limitations. The adoption of ERAS could have been underestimated, because not all elements could be collected retrospectively. It is possible that we had inadequate power to detect significant differences. However, given the $95 \%$ CIs, we would have been able to detect a clinically relevant effect of more than one day in the time to FR and TLOS favouring the intervention group [43].

In conclusion, we found neither statistically significant nor clinically relevant differences in the time to FR, TLOS, or the degree of local implementation of ERAS, between the gynaecology departments of hospitals that previously followed a QI project for colorectal surgery and the hospitals that did not. Interdepartmental spread of universal evidence-based innovations within organisations seems to be restricted. Further research is required to provide potential solutions to promote knowledge sharing within hospital walls and to extend the initial positive effects of QI projects. 


\section{REFERENCES}

1. Riskin DJ, Longaker MT, Gertner M et al (2006) Innovation in surgery: a historical perspective. Ann Surg 244:686-693

2. Grimshaw JM, Eccles MP, Lavis JN et al (2012) Knowledge translation of research findings. Implement Sci 7:50

3. Grimshaw JM, Thomas RE, MacLennan G et al (2004) Effectiveness and efficiency of guideline dissemination and implementation strategies. Health Technol Assess 8:iiiiv: $1-72$

4. Institute for Healthcare Improvement (2003) The breakthrough series: IHI's collaborative model for achieving breakthrough improvement. IHI Innovation Series White Paper, Institute for Healthcare Improvement, Boston

5. Kilo CM (1998) A framework for collaborative improvement: lessons from the Institute for Healthcare Improvement's Breakthrough Series. Qual Manag Health Care 6:1-13

6. Ovretveit J, Klazinga N (2012) Learning from large-scale quality improvement through comparisons. Int J Qual Health Care 24:463-469

7. Slaghuis SS, Strating MM, Bal RA et al (2013) A measurement instrument for spread of quality improvement in healthcare. Int J Qual Health Care 25:125-131

8. Massoud MR, Nielsen GA, Nolan K et al (2006) A framework for spread: from local improvements to system-wide change. IHI Innovation Series white paper, Institute for Healthcare Improvement, Cambridge

9. Greenhalgh T, Robert G, Macfarlane F et al (2004) Diffusion of innovations in service organizations: systematic review and recommendations. Milbank Q 82:581-629

10. Tasselli S (2014) Social networks of professionals in health care organizations: a review. Med Care Res Rev 71:619-660

11. Currie G, Waring J, Finn R (2008) The limits of knowledge management for UK public services modernization: the case of patient safety and service quality. Public Adm 86:363-385

12. Pearson ML, Upenieks VV, Yee T et al (2008) Spreading nursing unit innovation in large hospital systems. J Nurs Adm 38:146-152

13. Gillissen F, Hoff C, Maessen JM et al (2013) Structured synchronous implementation of an enhanced recovery program in elective colonic surgery in 33 hospitals in The Netherlands. World J Surg 37:1082-1093

14. Maessen J, Dejong CH, Hausel J et al (2007) A protocol is not enough to implement an enhanced recovery programme for colorectal resection. Br J Surg 94:224-231

15. de Groot JJ, van Es LE, Maessen JM et al (2014) Diffusion of enhanced recovery principles in gynecologic oncology surgery: is active implementation still necessary? Gynecol Oncol 134:570-575

16. Gustafsson UO, Scott MJ, Schwenk W et al (2013) Guidelines for perioperative care in elective colonic surgery: enhanced recovery after surgery (ERAS) society recommendations. World J Surg 37:259-284

17. Ljungqvist O, Scott M, Fearon KC (2017) Enhanced recovery after surgery: a review. JAMA Surg 152:292-298 2354 World J Surg (2018) 42:2348-2355 123

18. Mortensen K, Nilsson M, Slim K et al (2014) Consensus guidelines for enhanced recovery after gastrectomy: enhanced recovery after surgery (ERAS) society recommendations. $\mathrm{Br}$ J Surg 101:1209-1229 
19. Lassen K, Coolsen MM, Slim K et al (2013) Guidelines for perioperative care for pancreaticoduodenectomy: enhanced recovery after surgery (ERAS) society recommendations. World J Surg 37:240-258

20. Cerantola Y, Valerio M, Persson B et al (2013) Guidelines for perioperative care after radical cystectomy for bladder cancer: enhanced recovery after surgery (ERAS) society recommendations. Clin Nutr 32:879-887

21. Nelson G, Altman AD, Nick A et al (2016) Guidelines for preand intra-operative care in gynecologic/oncology surgery: enhanced recovery after surgery (ERAS) society recommendations-part I. Gynecol Oncol 140:313-322

22. Nelson G, Altman AD, Nick A et al (2016) Guidelines for postoperative care in gynecologic/oncology surgery: enhanced recovery after surgery (ERAS) society recommendations-part II. Gynecol Oncol 140:323-332

23. Paton F, Chambers D, Wilson P et al (2014) Effectiveness and implementation of enhanced recovery after surgery programmes: a rapid evidence synthesis. BMJ Open 4:e005015

24. Benedetti-Panici P, Maneschi F, Cutillo G et al (1997) A randomized study comparing retroperitoneal drainage with no drainage after lymphadenectomy in gynecologic malignancies. Gynecol Oncol 65:478-482

25. Charoenkwan K, Kietpeerakool C (2014) Retroperitoneal drainage versus no drainage after pelvic lymphadenectomy for the prevention of lymphocyst formation in patients with gynaecological malignancies. Cochrane Database Syst Rev 6:CD007387

26. Franchi M, Trimbos JB, Zanaboni F et al (2007) Randomised trial of drains versus no drains following radical hysterectomy and pelvic lymph node dissection: a European organisation for research and treatment of cancer-gynaecological cancer group (EORTCGCG) study in 234 patients. Eur J Cancer 43:1265-1268

27. Jensen JK, Lucci JA 3rd, DiSaia PJ et al (1993) To drain or not to drain: a retrospective study of closed-suction drainage following radical hysterectomy with pelvic lymphadenectomy. Gynecol Oncol 51:46-49

28. Lopes AD, Hall JR, Monaghan JM (1995) Drainage following radical hysterectomy and pelvic lymphadenectomy: dogma or need? Obstet Gynecol 86:960-963

29. Ahmed J, Khan S, Gatt M et al (2010) Compliance with enhanced recovery programmes in elective colorectal surgery. Br J Surg 97:754-758

30. Gustafsson UO, Hausel J, Thorell A et al (2011) Adherence to the enhanced recovery after surgery protocol and outcomes after colorectal cancer surgery. Arch Surg 146:571-577

31. Kahokehr A, Sammour T, Zargar-Shoshtari K et al (2009) Implementation of ERAS and how to overcome the barriers. Int J Surg 7:16-19

32. de Groot JJ, Ament SM, Maessen JM et al (2016) Enhanced recovery pathways in abdominal gynecologic surgery: a systematic review and meta-analysis. Acta Obstet Gynecol Scand 95:382-395

33. Barber EL, Van Le L (2015) Enhanced recovery pathways in gynecology and gynecologic oncology. Obstet Gynecol Surv 70:780-792

34. Nelson G, Kalogera E, Dowdy SC (2014) Enhanced recovery pathways in gynecologic oncology. Gynecol Oncol 135:586-594

35. Labgaa I, Jarrar G, Joliat GR et al (2016) Implementation of enhanced recovery (ERAS) in colorectal surgery has a positive impact on non-ERAS liver surgery patients. World J Surg 40:1082-1091 
36. Johnson MJ, May CR (2015) Promoting professional behaviour change in healthcare: what interventions work, and why? A theory-led overview of systematic reviews. BMJ Open 5:e008592

37. Vos L, Chalmers SE, Duckers ML et al (2011) Towards an organisation-wide processoriented organisation of care: a literature review. Implement Sci 6:8

38. Waring J, Currie G, Crompton A et al (2013) An exploratory study of knowledge brokering in hospital settings: facilitating knowledge sharing and learning for patient safety? Soc Sci Med 98:79-86

39. Bornbaum CC, Kornas K, Peirson L et al (2015) Exploring the function and effectiveness of knowledge brokers as facilitators of knowledge translation in health-related settings: a systematic review and thematic analysis. Implement Sci 10:162

40. Maessen JM, Dejong CH, Kessels AG et al (2008) Length of stay, an inappropriate readout of the success of enhanced recovery programs. World J Surg 32:971-975

41. Fiore JF Jr, Faragher IG, Bialocerkowski A et al (2013) Time to readiness for discharge is a valid and reliable measure of shortterm recovery after colorectal surgery. World J Surg 37:2927-2934

42. Gonc,alves-Bradley DC, Lannin NA, Clemson LM et al (2016) Discharge planning from hospital. Cochrane Database Syst Rev 1:CD000313

43. Goodman SN, Berlin JA (1994) The use of predicted confidence intervals when planning experiments and the misuse of power when interpreting results. Ann Intern Med 121:200-206 




\section{Diffusion of Enhanced Recovery principles in gynecologic oncology surgery \\ Is active implementation still necessary?}

Jeanny J.A. de Groot

Lilian E.J.M. van Es

José M.C. Maessen

Cornelis H.C. Dejong

Roy F.P.M. Kruitwagen

Brigitte F.M. Slangen

Gynecol oncol 2014; 134:570-575 


\begin{abstract}
Objective Spontaneous diffusion of the evidence-based Enhanced Recovery After Surgery (ERAS) program from an early adopter department (colorectal surgery) to other closely related departments (gynecologic surgery) within the same hospital could be expected. Given this diffusion hypothesis, this quality improvement study examines the value of active implementation of ERAS in addition to spontaneous diffusion.
\end{abstract}

Methods A nonrandomized, pre-post intervention study was conducted at a tertiary referral hospital. Prospective data of consecutive patients who underwent abdominal surgery between March, 2010 and March, 2011 for gynecologic malignancies were collected and compared with those of a historical cohort of patients treated before the structured implementation of ERAS by an expert team. Outcomes were length of hospital stay, length of functional recovery, and compliance to protocol care elements.

Results Seventy-seven patients treated after structured implementation of ERAS were compared with 38 patients included in the historical cohort. Most women had surgery for ovarian or endometrial cancer (48\% and 37\% respectively). Postoperative care mostly lacked ERAS elements and needed to be actively implemented. With structured implementation, a reduced time to functional recovery (median 3 versus 6 days, $\mathrm{p}<0.001$ ) and a shorter length of hospital stay ( 5 versus 7 days, $\mathrm{p}<0.001$ ) were achieved.

Conclusions After several years of practicing ERAS in colorectal surgery, spontaneous spread of ERAS principles to gynecologic oncology surgery occurred partially. The results of this study underscore the need for a structured and supported proactive process to implement the ERAS program in a complete and successful way. 


\section{BACKGROUND}

A major challenge in healthcare is to incorporate innovations into routine clinical practice. Many innovations require an intensive, well-planned approach to become widely adopted $[1,2]$. Although it usually takes many years to change well-established care, spontaneous spread of innovations can occur. Innovativeness is the essential factor in this process $[3,4]$. Apart from differences in diffusion rate, the completeness of adoption and the adherence in clinical practice also vary $[2,5]$. The multimodal Enhanced Recovery After Surgery (ERAS) program aims at a more evidence-based perioperative care and challenges the change in routine clinical practice [6]. ERAS was introduced to facilitate improvement of health care quality in elective colorectal surgery [7]. The program aims to minimize surgical stress by maintaining normal physiology to the best possible extent. As a consequence, postoperative recovery quickens and length of hospital stay decreases [6, 8-10]. The ERAS program consists of several preoperative, intraoperative, and postoperative elements and is currently general practice in colorectal surgery [10]. Because of positive results without additional morbidity or mortality, the program is gaining ground in other surgical fields such as orthopedics, thoracic surgery, urology, and gynecology [9, 11]. However, no randomized controlled trials have been conducted in gynecologic surgery in general [12], although favorable evidence has been provided by several prospective and retrospective studies for both malignant and benign pathologies [13-20].

In the Netherlands, the introduction of the ERAS guideline was pioneered by the surgery department of Maastricht University Medical Centre (MUMC). The department participated in a collaboration of five European University or specialized hospitals in 2001 to develop, introduce, and evaluate a new evidence-based program with 19 key recommendations [21]. This program was based on the original multimodal concept of Kehlet [7]. In 2006, a breakthrough project was initiated to achieve nationwide implementation of the ERAS protocol in 33 colorectal surgery departments [22]. Although several studies performed in colorectal surgery reported that consistent implementation of ERAS in clinical practice is difficult $[6,23,24]$, spontaneous uptake of ERAS guidelines in gynecology could be expected after several years. This could result from the nationwide positive results in colorectal surgery, the strong collaboration between clinicians in colorectal and gynecologic surgery, the involvement of the same anesthesiologists during operative procedures, and sometimes mixed surgical wards. Furthermore, the awareness of the need for high-quality perioperative care has increased in general. This raises the question of whether active implementation of ERAS is still necessary. This quality improvement study examines the level of spontaneous diffusion of ERAS in gynecologic oncology surgery and the additional value of a pro-active implementation process on the adoption of ERAS in clinical practice. 


\section{METHODS}

\section{Study design}

A single center, non-randomized, quality improvement study was conducted at the Department of Gynecology of Maastricht University Medical Centre in the Netherlands. This tertiary referral hospital is one of the Northern-European hospitals that pioneered the implementation of the ERAS guideline within the Department of Colorectal Surgery in 2001 [25] and is identified as one of the eleven centers of excellence of the ERAS Society (www.erassociety.org). A pre-post intervention design was used to assess the level of spontaneous uptake of ERAS elements and to examine the impact of active implementation on the adoption of the evidence-based ERAS program in gynecologic oncology surgery. After active implementation of ERAS at the Department of Gynecology in 2009, a prospective cohort of consecutive patients undergoing gynecologic surgery between March, 2010 and March, 2011 (post-implementation group) was compared with a historical cohort treated between January, 2007 and January, 2008 (pre-implementation group), before the ERAS program was actively introduced in gynecologic surgery. To exclude the possible influence of preparation activities, the one year period before implementation was randomly chosen as the period in which implementation of the ERAS program was not yet discussed. Considering the learning effect and to guarantee management according to a fully implemented program, analysis of the post-implementation study group was started six months after the initiation of the structured implementation of ERAS. The web-based Alberta Research Ethics Community Consensus Initiative (ARECCI) ethics screening tool for quality improvement research projects was used to determine the appropriate ethics review requirements [26]. Based on the assigned category of minimal risk, the study was exempted from institutional review board approval.

\section{Patient population}

All women aged 18 or over who underwent abdominal surgery through a transverse or midline incision for suspected or diagnosed ovarian, endometrial, or cervical cancer were included consecutively during the two predetermined study periods. Patients underwent operative cytoreduction or (radical) hysterectomy with bilateral salpingo-oophorectomy with and without pelvic lymphadenectomy. No exclusion criteria for participation in this study were used.

\section{Implementation strategy}

In October, 2009, the ERAS guideline [21] was actively introduced at the Department of Gynecology for patients undergoing major abdominal surgery. The implementa- 
tion was directed by a multidisciplinary expert team who had already implemented the ERAS guideline for colorectal surgery in the Netherlands. This team involved a surgeon, an anesthesiologist, and an implementation expert. The expert team was completed with two gynecologic oncologists, a nurse practitioner, and the director of nursing of the gynecologic department. The team educated other professionals who were involved in the perioperative period and was responsible for the dissemination, execution, and evaluation of the implementation process. Monthly audit and feedback sessions were organized to evaluate daily practice and to add further refinements for the optimization of perioperative care.

\section{Pre-implementation group}

Perioperative care in the historical 2007-2008 cohort group, before the supported implementation of the ERAS guideline in gynecology, was based on spontaneous diffusion of ERAS elements from colorectal surgery and was not explicitly established in protocols. In essence, it depended more on the personal preferences of the responsible gynecologist. Providing thromboembolic and antimicrobial prophylaxis to operative patients was already standard practice.

\section{Post-implementation group}

Patients in the post-implementation group were managed in accordance with the ERAS protocol using a previously described, evidence-based perioperative pathway [21]. The protocol consisted of extensive preoperative counseling, no preoperative oral fluid restriction, no bowel preparation, and carbohydrate loading up to two hours before surgery. Long acting anesthetics and opioids were avoided, and a thoracic epidural catheter was additionally used for analgesia. Use of drains and nasogastric tubes was limited. In the postoperative period early mobilization and oral intake were stimulated. The implemented elements of the ERAS protocol are described in more detail in Supplementary Table 1 (Table S1).

\section{Outcome measures}

The primary endpoint with respect to the successful adoption of ERAS management was the length of hospital stay, defined as the number of nights a patient stayed in the hospital after surgery. If readmission occurred within 30 days after surgery, the days of readmission were included in the total length of hospital stay. Secondary outcome measures were length of functional recovery and compliance to selected ERAS protocol elements. Functional recovery was achieved when patients tolerated a normal diet, mobilized independently and were comfortable on oral analgesia. The protocol elements scored were avoidance of preoperative bowel preparation, use of epidural anesthesia, avoidance of nasogastric tubes or removal at the end of 
surgery, early nutrition, and early mobilization. The selected elements were readily recordable for retrospective data collection as well, except for early mobilization. Early nutrition and early mobilization are key-elements in multivariate analyses and are important predictors of an enhanced recovery and reduced length of stay $[14,22,27,28]$. Achieving those two postoperative key-elements also depends on the degree of success of the adoption and adherence to other ERAS elements [28]. Early nutrition was defined as oral fluid intake on the day of the operation and normal oral intake on the first postoperative day. Early mobilization started on the day of surgery and was intensified during the first postoperative day.

\section{Data collection}

Data were prospectively collected in the post-implementation group. In the historical pre-implementation cohort, all data were retrospectively retrieved from medical records. Collected data included patient characteristics, procedure information, and readmission rates in both patient groups for 30 days after surgery. Additional data recorded were length of hospital stay, length of functional recovery, and adherence to the selected ERAS protocol elements. Patient privacy was protected by coding and processing all data anonymously. Follow-up was completed for all patients.

\section{Analysis}

Categorical data were presented as absolute counts with percentages, and continuous data were presented as median with interquartile range or as mean and standard deviation. Associations between groups were assessed using the Chi-square test or Fisher's exact test for small cohorts as appropriate. For continuous variables, the Mann-Whitney U test was used. The length of functional recovery and length of hospital stay in both study groups were compared using Kaplan-Meier curves and log-rank tests. Cox proportional hazard regression models were used to adjust for potential confounding variables [29]. The unadjusted and adjusted effects of the implementation of guidelines on length of hospital stay were compared to determine the effect of a single variable. If a variable changed the effect by at least $10 \%$, it was considered a confounder [29]. All confounding variables were included in the modeling process. Results were reported as hazard ratio (HR), 95\% confidence interval $(\mathrm{CI})$, and associated $p$-value. Associations were considered statistically significant if $\mathrm{p}<0.05$. Statistical analyses were performed using SPSS ${ }^{\circ}$ version 21 software (SPSS, Chicago, Illinois, USA). 
Table 1. Patient characteristics and procedure information.

\begin{tabular}{|c|c|c|c|}
\hline & $\begin{array}{l}\text { Pre implementation } \\
\mathrm{n}=38\end{array}$ & $\begin{array}{l}\text { Post implementation } \\
\mathrm{n}=77\end{array}$ & $p$-value \\
\hline Age in years, mean $( \pm S D)$ & $62.7(12.6)$ & $60.8(14.7)$ & $0.498^{\mathrm{a}}$ \\
\hline ASA grade, $\mathrm{n}(\%)$ & & & $0.276^{\mathrm{b}}$ \\
\hline $\mathrm{I} / \mathrm{II}$ & $27(71)$ & $65(84)$ & \\
\hline III & $8(21)$ & $11(14)$ & \\
\hline Missing & $3(8)$ & $1(1)$ & \\
\hline Diagnosis, n (\%) & & & $0.127^{\mathrm{b}}$ \\
\hline Ovarian cancer & $17(45)$ & $38(49)$ & \\
\hline Endometrial cancer & $18(47)$ & $24(31)$ & \\
\hline Cervical cancer & $3(8)$ & $15(20)$ & \\
\hline Type of incision, n (\%) & & & $0.059^{c}$ \\
\hline Midline & $31(82)$ & $72(94)$ & \\
\hline Transverse & $7(18)$ & $5(6)$ & \\
\hline Anastomosis, n (\%) & $1(3)$ & $2(3)$ & $1.000^{\mathrm{c}}$ \\
\hline
\end{tabular}

SD standard deviation; ASA American Society of Anesthesiologists.

${ }^{\mathrm{a}}$ Unpaired t test, ${ }^{\mathrm{b}}$ Chi-square test, ${ }^{\mathrm{c}}$ Fisher's exact test

\section{RESULTS}

\section{Patient characteristics}

A total of 115 patients were included in the study, of which 77 were in the postimplementation group and 38 in the pre-implementation group. Baseline characteristics and procedure information are presented in Table 1. Mean age was 62.7 years (standard deviation 12.6) in the pre-implementation group and 60.8 years (standard deviation 14.7) in the post-implementation group. The ovary and endometrium were the most common sites of pathology. Patient age, American Society of Anesthesiologists (ASA) grade, diagnosis, type of incision, and anastomosis rate did not differ between the two study groups $(p>0.05)$.

\section{Compliance to ERAS protocol elements}

Some of the ERAS elements had already been partially adopted into clinical practice before implementation activities were undertaken. In most patients of the preimplementation group, epidural anesthesia was used and bowel preparation and nasogastric tubes were omitted (Table 2). After implementation, the degree of adherence improved significantly for all registered aspects, except for the use of epidural anesthesia (66\% versus $65 \%, p=0.928$ ). In the pre-implementation group none of the patients started with oral fluids on the day of operation and none had normal oral intake on the first postoperative day. After implementation, the adherence rate to 
both postoperative elements increased to $94 \%$ and $58 \%$ respectively $(p<0.001)$. In $16 \%$ of the patients in the post-implementation group, mobilization started on the day of surgery in accordance with the ERAS protocol. Seventy-seven percent of the patients mobilized three times or more during the first postoperative day. The early mobilization data could not be retrieved for the pre-implementation group, whereas the independent mobility data could.

Table 2. Comparison of compliance to ERAS protocol elements.

\begin{tabular}{llll}
\hline & $\begin{array}{l}\text { Pre implementation } \\
\mathbf{n}=38\end{array}$ & $\begin{array}{l}\text { Post implementation } \\
\mathbf{n}=77\end{array}$ & $\begin{array}{c}\text { P-value } \\
\text { Avoidance of preoperative bowel preparation }\end{array}$ \\
$35(92)$ & $77(100)$ & $<0.05^{\mathrm{a}}$ \\
\hline Epidural anesthesia & $25(66)$ & $50(65)$ & $0.928^{\mathrm{b}}$ \\
\hline Avoidance of nasogastric tubes & $33(87)$ & $77(100)$ & $<0.05^{\mathrm{a}}$ \\
\hline Oral fluid on day 0 & $0(0)$ & $72(94)$ & $<0.05^{\mathrm{b}}$ \\
\hline Normal food on day 1 & $0(0)$ & $45(58)$ & $<0.05^{\mathrm{b}}$ \\
\hline
\end{tabular}

Values expressed as numbers (percentages).

a Fisher's exact test, b Chi-square test

\section{Length of functional recovery}

In the post-implementation group, patients had shorter median times to tolerate oral fluid and food intake, were sooner comfortable on oral analgesia, and reached independent mobility faster (Table 3). Based on fulfilling these criteria, functional recovery was achieved three days earlier after implementation of the ERAS protocol. The median time to functional recovery was 6 days (interquartile range, $5-7$ days) in the pre-implementation group compared with 3 days (interquartile range, $3-4.5$ days) in the post-implementation group ( $\mathrm{p}<0.001$, Table 4$)$.

\section{Length of hospital stay}

The median length of hospital stay was significantly reduced after implementation of the ERAS criteria ( $\mathrm{p}<0.001$, Fig. 1). Patients enrolled in the ERAS program were discharged after 5 days (interquartile range, 4-7 days), whereas the median length of hospital stay was 7 days (interquartile range, 6-9.25 days) in the pre-implementation group (Table 4). Fifty-five percent of the patients $(n=43)$ in the post-implementation group had a length of hospital stay of 5 days or less, compared to $13 \%(n=5)$ in the pre-implementation group $(\mathrm{p}<0.001)$. Both before and after implementation, one patient was readmitted to the hospital. This did not influence median hospital stay or other outcome measures. None of the patients died during the first month after operation. Cox regression analysis was performed with age, ASA score, type of incision, and the presence of intestinal anastomosis as potential confounding factors. Because none of these factors influenced the effect of implementation by 
Table 3. Achievement of recovery criteria in postoperative days.

\begin{tabular}{llll}
\hline & Pre implementation & Post implementation & $p$ value $^{\text {a }}$ \\
\hline Intake of oral fluids & $2(2-3)$ & $0(0-0)$ & $<0.001$ \\
\hline Normal food intake & $3(3-4)$ & $1(1-2)$ & $<0.001$ \\
\hline Independent mobility & $6(5-7)$ & $3(3-4.5)$ & $<0.001$ \\
\hline Comfortable on oral analgesia & $3(2-4)$ & $2(2-3)$ & $<0.001$ \\
\hline
\end{tabular}

Values expressed as median (interquartile range).

a Mann-Whitney U test

Table 4. Influence of implementation of the ERAS program on length of recovery and total length of hospital stay.

\begin{tabular}{lll}
\hline & Pre implementation & Post implementation \\
\hline Length of recovery & 38 & 77 \\
\hline Patients, number & $6(5-7)$ & $3(3-4.5)$ \\
\hline Median days $(\mathrm{IQR})$ & $6.7( \pm 3.2)$ & $3.9( \pm 1.7)$ \\
\hline Mean days $( \pm \mathrm{SD})$ & & $2.6(1.7-3.9)$ \\
\hline Hazard ratio $(95 \% \mathrm{CI})$ & & $<0.001$ \\
\hline$p$-value & 38 & 77 \\
\hline Total length of hospital stay & $7(6-9.25)$ & $5(4-7)$ \\
\hline Patients, number & $9.5( \pm 6.4)$ & $5.7( \pm 2.0)$ \\
\hline Median days $(\mathrm{IQR})$ & & $2.3(1.5-3.5)$ \\
\hline Mean days $( \pm \mathrm{SD})$ & & $<0.001$ \\
\hline Hazard ratio $(95 \% \mathrm{CI})$ & & \\
\hline$p$-value & & \\
\hline
\end{tabular}

IQR interquartile range; SD standard deviation; CI confidence interval

more than $10 \%$, they were not considered as confounders of the observed association between a reduced total length of hospital stay and the implementation of ERAS. The unadjusted hazard ratio for the effect of implementation of ERAS in patients with malignant gynecologic pathology was 2.3 (95\% CI, 1.5-3.5). The corresponding p-value was $<0.001$ (Table 4). The hazard ratio of 2.3 corresponds to a $70 \%$ chance of a patient in the post-implementation group being discharged first compared with a patient in the pre-implementation group. We observed a gap between functional recovery and actual discharge, defined as delayed discharge, both before $(\mathrm{n}=29$, $76 \%$ ) and after ( $\mathrm{n}=66,86 \%$ ) implementation of the ERAS program. The length of delayed discharge did not differ between the two groups $(\mathrm{p}=0.097)$. Median length of delayed discharge was 2 days before (interquartile range, 1-4 days) and 2 days after ERAS implementation (interquartile range, 1-2.25 days). 


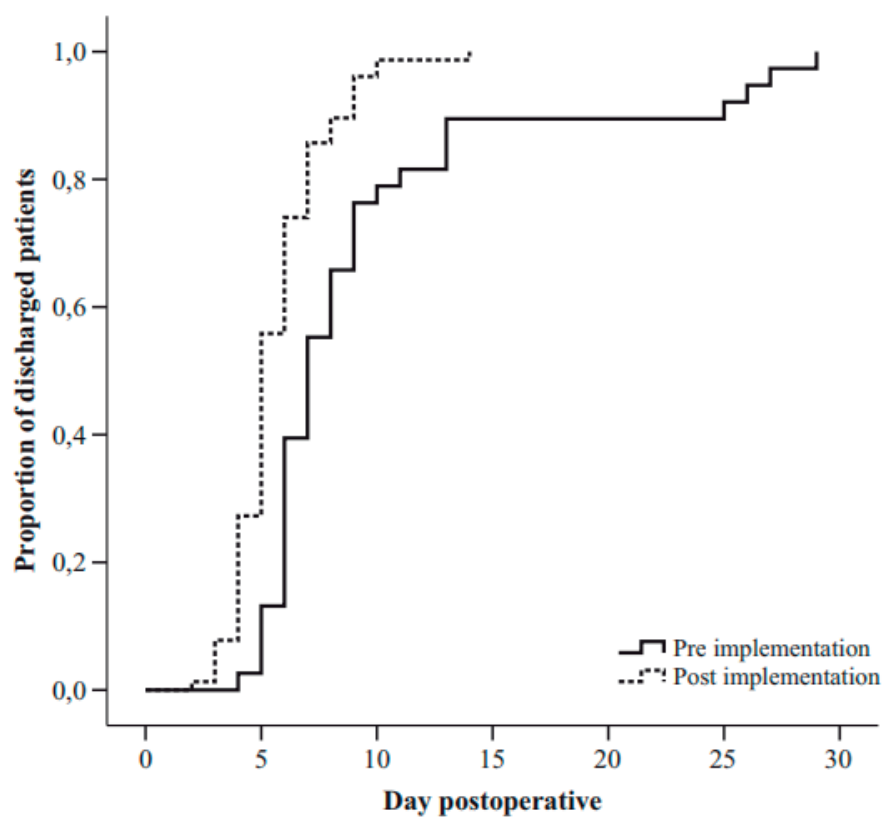

Figure 1. Kaplan Meier curves of total length of hospital stay in the pre-implementation and postimplementation groups.

\section{DISCUSSION}

The results of the present study indicate that after several years of practicing ERAS in colorectal surgery, its implementation in major gynecologic surgery has lagged within the same hospital. Spontaneous spread and natural uptake of the program from the early adopter surgical department to another related department occurred partially. The pre- and intraoperative elements of the ERAS program have largely been adopted in standard practice. Disuse of bowel preparation, additional use of epidural anesthesia, and avoidance of nasogastric tubes were applied in more than two-thirds of the patients treated before structured implementation. Adoption of the postoperative key-elements in daily practice, on the contrary, did not occur naturally. The present results suggest that barriers between the gynecologic and colorectal surgery departments are still larger than expected. Complete implementation of the evidence-based ERAS program in gynecology requires an active and organized approach.

The involvement of gynecologists, anesthesiologists, and colorectal surgeons during major surgical procedures encourages interdisciplinary collaboration and sharing of best practices [30]. This could have contributed to the spread of preoperative and intraoperative elements. Unlike medical specialists, nurses have limited 
exposure to interdisciplinary collaboration. Nursing practice on wards remains separate from that of other surgical fields. Over the last decade, evidence-based nursing has gained increasing interest. Although the integration of prevailing evidence has been extended to nursing care, routine evidence-based nursing still lags behind [31]. Traditionally, nursing practice is based more on habits and expertise than on evidence. Several studies have identified individual and organizational barriers to nurses' utilization of research in general [32-34]. Those universal factors are likely to be applicable to the lack of natural uptake of postoperative ERAS elements. The observed lack of natural uptake could also be attributed to the fact that postoperative ERAS elements directly reflect the condition of patients after surgery and that their application cannot be forced [35]. Apparently, the pre- and intraoperative elements are easier to implement. Changing the mindset of health care providers and patients in the postoperative care setting seems to be more challenging [36]. Several studies have shown the safety of early mobilization and intake [7, 14, 21, 37, 38]. However, the deep-rooted feeling that this could be harmful for patients' health, might delay recovery and prolong hospital stay unnecessarily.

In the present study, we focused on perioperative management of abdominal surgery in patients with gynecologic cancer because of the known benefits of ERAS for those procedures [14-18]. The median length of hospital stay in our study was reduced to five days, which is the upper limit of the median length of stay reported by fast track perioperative care studies in gynecologic oncology surgery (range, 2-5 days) [14-18]. Patients recover earlier when treated in line with the ERAS program. Given the substantial variation in adherence to the postoperative elements, early nutrition and mobilization, our data confirm that those key-elements may be crucial for the success of ERAS [14, 22, 27, 28]. In practice, it seemed harder to follow the guideline strictly. Unlike the compliance with oral fluid intake on the day of surgery, the avoidance of bowel preparation, and nasogastric tubes ( $>90 \%)$, we observed that use of epidural anesthesia (65\%) and normal oral intake on the first postoperative day (58\%) were not standard practice for all patients. Gillissen et al. [22] found comparable compliance rates after implementing ERAS in colorectal surgery (56 - 96\%). Even after structured implementation, compliance with protocol elements remains challenging [24, 28, 35, 39], and the continuous evolvement of new evidence further challenges evidence-based practice [40]. Despite this deviation in one or more elements of the ERAS protocol, our study once more demonstrates that implementation of an ERAS program leads to faster recovery and earlier discharge [24].

Because of the known benefit of ERAS programs regarding recovery and given the fact that running two different postoperative care protocols within one department is prone to bias, a pre-post intervention design was used. Furthermore, the focus of the study was not to demonstrate the beneficial effects of ERAS on recovery, but 
rather to examine the need for a structured and supported implementation process. However, the historical design of the study is a limitation. As a consequence, not all elements could be retrieved in the retrospective cohort, and a selection of outcome measures was required. Bias was reduced by using the same criteria for discharge and functional recovery in both study groups. It could be expected that in the prospective study group, awareness of prospective data collection could have influenced decisions on discharge, irrespective of functional recovery. However, the gap between recovery and actual discharge did not differ between the two study groups. Therefore, the effect on outcome measures is assumed to be minimal. Because randomization was not feasible, adjustment for the effect of confounding variables was applied at the stage of data analysis. During the four years between the start of the retrospective study and the end of the prospective study, a natural evolution of perioperative care could have occurred, independent of the ERAS implementation project. However, in the six-year period after the introduction of ERAS at the surgery department in our hospital, only certain ERAS elements were partially adopted in our retrospective study group. Therefore, we believe that the effect in the four-year period is small. The difference in the number of patients included in each study period (38 and 77) was caused by an increase in patient load. Both study periods lasted one year and all consecutive patients were included in the study. The increase in patient load could partially be explained by the ongoing centralization of major gynecologic surgery in the Netherlands.

This study underlines the necessity of a pro-active process to implement the ERAS program in a complete and successful way. Despite the fact that our hospital is one of the centers of excellence of the ERAS Society and had a pioneering role in the nationwide implementation of ERAS in colorectal surgery, spontaneous spread of ERAS principles among departments did not happen satisfactorily, primarily during the postoperative period. Transmission of new evidence-based practice between departments within the same hospital and overcoming apparent barriers remain challenging. The appropriate implementation of the ERAS program could lead to a higher level of perioperative care. 


\section{REFERENCES}

1. Grimshaw JM, Eccles MP, Walker AE, Thomas RE. Changing physicians' behavior: what works and thoughts on getting more things to work. J Contin Educ Health Prof 2002;22(4):237-43.

2. Grol R, Grimshaw J. From best evidence to best practice: effective implementation of change in patients' care. Lancet 2003;362(9391):1225-30. doi:10.1016/S01406736(03)14546-1.

3. Berwick DM. Disseminating innovations in health care. JAMA 2003;289(15):1969-75.

4. Rogers E. Diffusion of Innovations. 5th ed. New York: Free Press; 2003.

5. Cabana MD, Rand CS, Powe NR, Wu AW, Wilson MH, Abboud PA, Rubin HR. Why don't physicians follow clinical practice guidelines? A framework for improvement. JAMA 1999;282(15):1458-65.

6. Kehlet H, Wilmore DW. Evidence-based surgical care and the evolution of fast-track surgery. Ann Surg 2008;248(2):189-98.

7. Kehlet H. Multimodal approach to control postoperative pathophysiology and rehabilitation. Br J Anaesth 1997;78(5):606-17.

8. Spanjersberg WR, Reurings J, Keus F, van Laarhoven CJ. Fast track surgery versus conventional recovery strategies for colorectal surgery. Cochrane Database Syst Rev 2011;2:CD007635.

9. Ansari D, Gianotti L, Schroder J, Andersson R. Fast-track surgery: procedure-specific aspects and future direction. Langenbecks Arch Surg 2013;398(1):29-37.

10. Zhuang CL, Ye XZ, Zhang XD, Chen BC, Yu Z. Enhanced recovery after surgery programs versus traditional care for colorectal surgery: a meta-analysis of randomized controlled trials. Dis Colon Rectum 2013;56(5):667-78.

11. Gravante G, Elmussareh M. Enhanced recovery for non-colorectal surgery. World J Gastroenterol 2012;18(3):205-11.

12. Lu D, Wang X, Shi G. Perioperative enhanced recovery programmes for gynaecological cancer patients. Cochrane Database Syst Rev 2012;12:CD008239.

13. Borendal Wodlin N, Nilsson L. The development of fast-track principles in gynecological surgery. Acta Obstet Gynecol Scand 2013;92(1):17-27.

14. Carter J. Fast-track surgery in gynaecology and gynaecologic oncology: a review of a rolling clinical audit. ISRN Surg 2012;2012:368014.

15. Kalogera E, Bakkum-Gamez JN, Jankowski CJ, Trabuco E, Lovely JK, Dhanorker S, Grubbs PL, Weaver AL, Haas LR, Borah BJ, Bursiek AA, Walsh MT, Cliby WA, Dowdy SC. Enhanced recovery in gynecologic surgery. Obstet Gynecol 2013;122(2 Pt 1):319-28.

16. Sidhu VS, Lancaster L, Elliott D, Brand AH. Implementation and audit of 'Fast-Track Surgery' in gynaecological oncology surgery. Aust N Z J Obstet Gynaecol 2012;52(4):3716.

17. Chase DM, Lopez S, Nguyen C, Pugmire GA, Monk BJ. A clinical pathway for postoperative management and early patient discharge: does it work in gynecologic oncology? Am J Obstet Gynecol 2008;199(5):541 e1-7.

18. Marx C, Rasmussen T, Jakobsen DH, Ottosen C, Lundvall L, Ottesen B, Callesen T, Kehlet $\mathrm{H}$. The effect of accelerated rehabilitation on recovery after surgery for ovarian malignancy. Acta Obstet Gynecol Scand 2006;85(4):488-92. 
19. Dickson E, Argenta PA, Reichert JA. Results of introducing a rapid recovery program for total abdominal hysterectomy. Gynecol Obstet Invest 2012;73(1):21-5.

20. Rardin CR, Weisman CJ, Kim YB, Niloff JM. Early discharge after abdominal surgery: experience on a gynecologic oncology service. Gynecol Oncol 1999;75(1):47-50.

21. Fearon KC, Ljungqvist O, Von Meyenfeldt M, Revhaug A, Dejong CH, Lassen K, Nygren J, Hausel J, Soop M, Andersen J, Kehlet H. Enhanced recovery after surgery: a consensus review of clinical care for patients undergoing colonic resection. Clin Nutr 2005;24(3):46677.

22. Gillissen F, Hoff C, Maessen JM, Winkens B, Teeuwen JH, von Meyenfeldt MF, Dejong CH. Structured synchronous implementation of an enhanced recovery program in elective colonic surgery in 33 hospitals in The Netherlands. World J Surg 2013;37(5):1082-93.

23. Aarts MA, Okrainec A, Glicksman A, Pearsall E, Victor JC, McLeod RS. Adoption of enhanced recovery after surgery (ERAS) strategies for colorectal surgery at academic teaching hospitals and impact on total length of hospital stay. Surg Endosc 2012;26(2):442-50.

24. Ahmed J, Khan S, Lim M, Chandrasekaran TV, MacFie J. Enhanced recovery after surgery protocols - compliance and variations in practice during routine colorectal surgery. Colorectal Dis 2011;14(9):1045-51.

25. Maessen J, Dejong CH, Hausel J, Nygren J, Lassen K, Andersen J, Kessels AG, Revhaug A, Kehlet H, Ljungqvist O, Fearon KC, von Meyenfeldt MF. A protocol is not enough to implement an enhanced recovery programme for colorectal resection. Br J Surg 2007;94(2):224-31.

26. ARECCI Ethics Screening Tool: developed by the Alberta Research Ethics Community Consensus Initiative (ARECCI) Network; (2005, revised 2008). Available from: http:/ www.ahfmr.ab.ca/arecci/screening.

27. Vlug MS, Bartels SA, Wind J, Ubbink DT, Hollmann MW, Bemelman WA. Which fast track elements predict early recovery after colon cancer surgery? Colorectal Dis 2011;14(8):1001-8.

28. Smart NJ, White P, Allison AS, Ockrim JB, Kennedy RH, Francis NK. Deviation and failure of enhanced recovery after surgery following laparoscopic colorectal surgery: early prediction model. Colorectal Dis 2012;14(10):e727-34.

29. Braga LH, Farrokhyar F, Bhandari M. Confounding: what is it and how do we deal with it? Can J Surg 2012;55(2):132-8.

30. Petri L. Concept analysis of interdisciplinary collaboration. Nurs Forum 2010;45(2):7382.

31. Thompson DS, Estabrooks CA, Scott-Findlay S, Moore K, Wallin L. Interventions aimed at increasing research use in nursing: a systematic review. Implement Sci 2007;2:15.

32. Fink R, Thompson CJ, Bonnes D. Overcoming barriers and promoting the use of research in practice. J Nurs Adm 2005;35(3):121-9.

33. Funk SG, Champagne MT, Wiese RA, Tornquist EM. Barriers to using research findings in practice: the clinician's perspective. Appl Nurs Res 1991;4(2):90-5.

34. Retsas A. Barriers to using research evidence in nursing practice. J Adv Nurs 2000;31(3):599-606.

35. Boulind CE, Yeo M, Burkill C, Witt A, James E, Ewings P, Kennedy RH, Francis NK. Factors predicting deviation from an enhanced recovery programme and delayed discharge after laparoscopic colorectal surgery. Colorectal Dis 2011;14(3):e103-10. 
36. Kahokehr A, Sammour T, Zargar-Shoshtari K, Thompson L, Hill AG. Implementation of ERAS and how to overcome the barriers. Int J Surg 2009;7(1):16-9.

37. Charoenkwan K, Phillipson G, Vutyavanich T. Early versus delayed (traditional) oral fluids and food for reducing complications after major abdominal gynaecologic surgery. Cochrane Database Syst Rev 2007(4):CD004508.

38. Gerardi MA, Santillan A, Meisner B, Zahurak ML, Diaz Montes TP, Giuntoli RL, 2nd, Bristow RE. A clinical pathway for patients undergoing primary cytoreductive surgery with rectosigmoid colectomy for advanced ovarian and primary peritoneal cancers. Gynecol Oncol 2008;108(2):282-6.

39. Feroci F, Lenzi E, Baraghini M, Garzi A, Vannucchi A, Cantafio S, Scatizzi M. Fast-track Surgery in Real Life: How Patient Factors Influence Outcomes and Compliance With an Enhanced Recovery Clinical Pathway After Colorectal Surgery. Surg Laparosc Endosc Percutan Tech 2013;23(3):259-65.

40. Hoffmann H, Kettelhack C. Fast-track surgery--conditions and challenges in postsurgical treatment: a review of elements of translational research in enhanced recovery after surgery. Eur Surg Res 2012;49(1):24-34. 


\section{SUPPLEMENTARY FILE}

Table S1. Description of implemented elements of the ERAS program.

\begin{tabular}{|c|c|c|}
\hline Timing & Element & Description \\
\hline \multirow[t]{5}{*}{ Preoperative } & Preoperative counseling & $\begin{array}{l}\text { Detailed information about the ERAS program during an } \\
\text { outpatient clinic appointment }\end{array}$ \\
\hline & Bowel preparation & No mechanical bowel preparation or just single rectal enema \\
\hline & Fasting & $\begin{array}{l}\text { Solids allowed up to six hours and clear fluids up to two hours } \\
\text { prior to operation }\end{array}$ \\
\hline & Carbohydrate treatment & $\begin{array}{l}\text { 400mL carbohydrate drink (PreOP®, Nutricia; Numico, } \\
\text { Zoetermeer, the Netherlands) two hours before operation, } \\
\text { unless diabetic }\end{array}$ \\
\hline & Sedative medication & $\begin{array}{l}\text { Not standard, only short-acting sedative medication permitted } \\
\text { if necessary }\end{array}$ \\
\hline \multirow[t]{4}{*}{ Intraoperative } & Epidural analgesia & $\begin{array}{l}\text { Mid-thoracic epidural analgesia, removal on postoperative day } \\
\text { two or three }\end{array}$ \\
\hline & Prevention of hypothermia & Standard use of a Bair hugger \\
\hline & Nasogastric tube & Not used, otherwise immediately removed after surgery \\
\hline & Abdominal drains & Not used \\
\hline \multirow[t]{6}{*}{ Postoperative } & Urinary catheter & Standard, removal at the same time as the epidural catheter \\
\hline & Analgesics & $\begin{array}{l}\text { Use of epidural analgesia with additional paracetamol from } \\
\text { day } 0\end{array}$ \\
\hline & Oral fluid intake & $\begin{array}{l}\text { Permitted immediately after surgery (day } 0 \text { ) } \\
\text { Intravenous fluids stopped on day } 1\end{array}$ \\
\hline & Normal diet & Offered on postoperative day 1 \\
\hline & Oral laxatives & 1000mg oral magnesium from day 1 till defecation \\
\hline & Early mobilization & $\begin{array}{l}\text { Sitting up on day } 0 \text { and intensified during the first } \\
\text { postoperative day (at least three times in chair) }\end{array}$ \\
\hline
\end{tabular}

Abbreviations: ERAS, enhanced recovery after surgery 




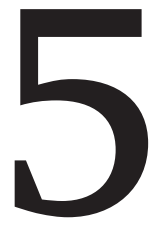

A stepped strategy that aims at the nationwide implementation of the Enhanced Recovery After Surgery programme in major gynaecological surgery

Study protocol of a cluster randomised controlled trial

Jeanny J.A. de Groot José M.C. Maessen Brigitte F.M. Slangen Bjorn Winkens Carmen D. Dirksen Trudy van der Weijden 


\begin{abstract}
Background Enhanced Recovery After Surgery (ERAS) programmes aim at an early recovery after surgical trauma and consequently at a reduced length of hospitalisation. This paper presents the protocol for a study that focuses on large scale implementation of the ERAS programme in major gynaecological surgery in the Netherlands. The trial will evaluate effectiveness and costs of a stepped implementation approach that is characterised by tailoring the intensity of implementation activities to the needs of organisations and local barriers for change, in comparison with the generic breakthrough strategy that is usually applied in large scale improvement projects in the Netherlands.
\end{abstract}

Methods All Dutch hospitals authorised to perform major abdominal surgery in gynaecological oncology patients are eligible for inclusion in this cluster randomised controlled trial. The hospitals that already fully implemented the ERAS programme in their local perioperative management or those who predominantly admit gynaecological surgery patients to an external hospital replacement care facility will be excluded. Cluster randomisation will be applied at hospital level and will be stratified based on tertiary status. Hospitals will be randomly assigned to the stepped implementation strategy or the breakthrough strategy. The control group will receive the traditional breakthrough strategy with three educational sessions and the use of plan-do-study-act cycles for planning and executing local improvement activities. The intervention group will receive an innovative stepped strategy comprising four levels of intensity of support. Implementation starts with generic low-cost activities and may build up to the highest level of tailored and labour-intensive activities. The decision for a stepwise increase in intensive support will be based on the success of implementation so far. Both implementation strategies will be completed within one year, and evaluated on effect, process, and cost-effectiveness. The primary outcome is length of postoperative hospital stay. Additional outcome measures are length of recovery, guideline adherence, and mean implementation costs per patient.

Discussion This study takes up the challenge to evaluate an efficient strategy for large scale implementation. Comparing effectiveness and costs of two different approaches, this study will help to define a preferred strategy for nationwide dissemination of best practices. 


\section{BACKGROUND}

Malignant neoplasms are the most important indication for major surgery in gynaecology in the Netherlands each year. Approximately 2500 women undergo gynaecological surgery for ovarian, endometrial, or cervical cancer. The last decades, several efforts have been made in trying to reach optimal perioperative management. In the late 1990s, Kehlet introduced a renewal process aiming to maintain normal physiology after surgery [1]. Several evidence-based elements were combined in a perioperative management programme, designed to enhance recovery from major surgical trauma [2]. The Enhanced Recovery After Surgery (ERAS) programme proved to be safe with favourable effects on recovery and length of stay [3-6]. Although this programme has been successfully implemented in elective colonic surgery, a spontaneous diffusion of the ERAS programme in other elective surgery areas did not occur [7-11]. Patients undergoing major surgical procedures remain subjected to unpleasant, unnecessary, and harmful practices; despite of a large body of evidence supporting perioperative management improvements [12-14]. As a result patients are exposed to an unnecessary prolonged hospital stay. Efficient implementation of the ERAS programme in elective gynaecological surgery on large scale is challenging.

In 2010 the ERAS programme was successfully introduced in major gynaecological surgery at the Department of Gynaecology of Maastricht University Medical Centre [11]. This resulted in a significant reduction in length of hospital stay. Nationwide implementation of the ERAS programme in elective gynaecological surgery did not happen yet, while this is necessary to achieve high standards of care and evidencebased practice, with subsequent benefits for the successful execution of clinical trials within this field $[15,16]$. Further, reduced hospitalisation will save costs and will facilitate the increased patient load by the imminent centralisation of major gynaecological surgery in the assigned high volume centres in the Netherlands. Finally, a reduced functional decline after surgery will ease the required early start of postoperative adjuvant chemotherapy in patients with ovarian cancer.

In the Netherlands, the quality improvement model known as the Breakthrough Series [17] is currently adopted as the preferred method for health care improvement on a large scale, despite some serious drawbacks [18]. This structured collaborative learning strategy is designed to help health care organizations attain best practice in a certain field. Systematic evaluations have shown that effectiveness of the breakthrough approach varies and is at the best moderate [19]. Furthermore, breakthrough projects represent substantial investments of time, effort, and funding and are assumed to be expensive [20]. A new approach designed to deliver an optimal effect of implementation efforts at the lowest possible implementation costs is desirable. Based on the underlying assumption that organisations may vary 
widely in their knowledge, motivation, and capacity to run a quality improvement programme, as prescribed in the meta-evaluation of ten such programmes in the Netherlands [21], a stepped implementation strategy was designed. This strategy aims to tailor the implementation efforts to the variety in needs and local barriers to change. It offers generic low-intensive support for those who smoothly implement the changes and a more tailored and intensive approach for those hospitals having difficulties in making the changes. This differentiation of the implementation activities to preparedness for change is described in various theories on the different phases in a process of change. The Model for Planning Change of Grol describes ten stages, subdivided in awareness, understanding, acceptance, adoption and integration of the proposed behaviour $[22,23]$. This model was used to select the change interventions that will be used in the stepped implementation strategy. Each step leads to a more intensive stage in the process of change. A tailored approach is intensive and seems less suitable for large scale improvement as it requires local support and elaborative preparatory activities [24, 25]. Evaluation of the process of this implementation effort will provide insight in why and for whom the developed strategy works and improves the understanding of the stepped approach. This will help to define a preferred strategy for nationwide dissemination of best practices.

\section{Objectives}

The objective of this study is to compare effectiveness and costs of a stepped implementation strategy versus the generic traditional breakthrough methodology for the nationwide uptake of the ERAS approach. In addition, the current study aims to identify intervention fidelity and contextual factors that influence implementation by means of a process evaluation. The overall aim is to define a preferred strategy for large scale implementation of evidence-based practice. This paper describes the study protocol.

\section{METHODS}

\section{Design}

The present study is a non-blinded cluster randomised controlled trial with two arms comparing an innovative stepwise implementation approach to the existing generic breakthrough approach. After approval to participate, a retrospective baseline measurement of 30 patients will be performed to assess current perioperative practice. Cluster randomisation will be applied at hospital level and will take place after all retrospective measurements have been completed. Randomisation will be executed at central level by an independent statistician of Maastricht University. The 
hospitals will be allocated to the intervention (stepped strategy) or to the control group (breakthrough strategy) (Fig. 1). After one year, the implementation process will be ended by a final sustainability meeting for both groups. We assume differences in uptake of innovations between the high-volume tertiary care hospitals and their affiliated regional hospitals. Stratified randomisation will be used to prevent unbalanced intervention groups with respect to tertiary status or not.

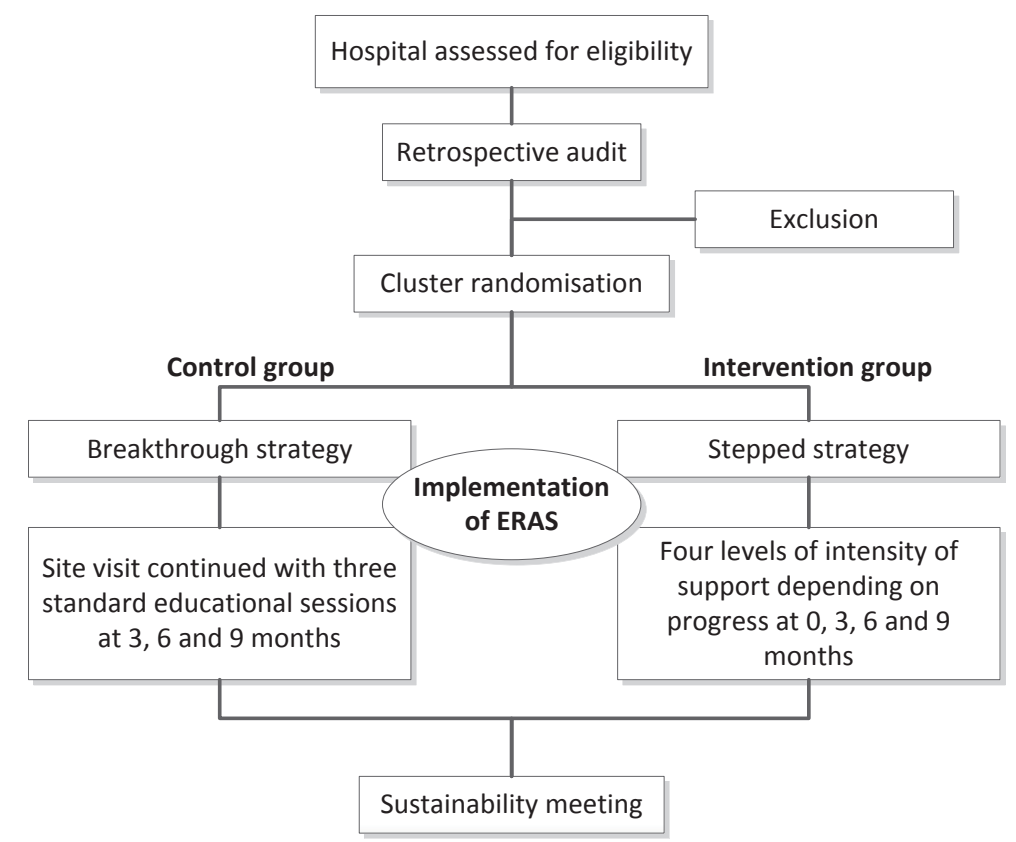

Figure 1. Flow chart SINERGY trial

\section{Participants}

Hospitals

All Dutch Gynaecology Oncology Group (DGOG) tertiary referral hospitals and their affiliated regional hospitals authorised to perform major gynaecological surgery will be invited to participate in this implementation study. The authorisation is based on the volume norm set by the Dutch Association of Gynaecologists in 2013. The hospitals that already fully implemented the ERAS programme in their local perioperative management are not eligible to participate. Exclusion will be based on findings of the baseline audit. To avoid interference in outcome measures, the hospitals that predominantly admit gynaecological surgery patients to an external hospital replacement care facility after discharge will be excluded as well. The DGOG, a national collaborative of professionals involved in the treatment of women with gynaecological cancers that aims to promote (inter)national research in gynae- 
cological cancer, supports the underlying aim to reach nationwide standardisation of perioperative care consistent with the ERAS programme. This will support the recruitment of eligible hospitals in the study. In addition, we expect that cancelation of the usual 20,000 euro fee for participation to a Dutch Institute for Healthcare Improvements (CBO)-guided breakthrough project will be an important incentive towards participation. For all participating hospitals, costs of implementation are brought back to costs of joining meetings and costs of executing local activities.

\section{Professionals}

The innovation focuses on a professional level, directed to individual hospitals and their key target groups. Key target groups are gynaecologists and anaesthetists involved in gynaecological surgery and nurses of the gynaecology department. Gynaecological oncologists of the tertiary hospitals assist in major ovarian surgery in the regional hospitals that are authorised to perform these procedures. These oncologists are technical experts assisting in the operating room and are not involved in pre and postoperative care. They are judged to have no influence on the implementation of the programme in the regional hospitals.

\section{Patients}

Women aged over 18 years and scheduled for elective abdominal surgery for diagnosis and/or treatment of (suspected) ovarian, endometrial, or cervical cancer will be included during the 1-year implementation period. No specific exclusion criteria will be applied.

\section{Control group}

Hospitals randomised to the control group will receive the traditional breakthrough strategy. The breakthrough series will be performed as usual. The CBO will chair the collaborative and recruit an expert panel including subject matter experts and an implementation expert. All participating hospitals will be instructed to appoint a multidisciplinary improvement team to lead the local improvement activities and will be visited by a CBO consultant and the researcher of the project to clarify the collaborative processes and expectations. Three educational sessions will be conducted with three months in between (Fig. 2). At the first learning session, the kick-off meeting, the expert team presents the vision of ideal care (the ERAS programme) and shows that actual care deviates from ideal care. The participants are taught an approach for organising and carrying out their improvement activities. This approach is called the Model for Improvement, and identifies four key elements of successful process improvement: measurable aims, measures of improvement, key changes that will result in the desired performance, and a series of testing 
cycles, known as plan-do-study-act (PDSA) cycles [26]. This model enables teams to test change locally and then reflect, learn and refine the changes. At the second and third learning session teams learn from each other as they report on successes by feeding back their data during the learning sessions, report on lessons learned, and exchange experiences.

\section{Intervention group}

The intervention group will be exposed to the stepped implementation strategy, which consists of four steps of implementation activities. This strategy is built on the assumptions that for some hospitals a generic improvement approach may be sufficient, while for other hospitals a full-blown breakthrough approach or an even more intensive tailored approach may be required. The four levels of intensity will be offered successively, with 3 months in between (Fig. 2).

\section{First implementation level}

The first implementation step aims at promoting awareness and comprises a digital toolbox available for the participating hospitals. The toolbox consists of background information of the ERAS programme, a short summary of the programme to use as a reminder in daily care, and a ready-to-use patient information sheet to structure patients' preadmission counselling and postoperative care. We assume that this toolbox might pave the way to start implementation for those clinical leaders who have the skills to guide their department through this innovation. This assumption is fed by our experience that in the project 'implementation of short stay in breast cancer surgery' [27] as well as in the breakthrough project 'perioperative care' [28] some hospitals started immediately with treating patients according to the new programme after the programme was disseminated.

\section{Second implementation level}

The second step is built on support to make change happen via an interactive learning session, provided by experts in the field of implementation and experts in the implementation of the ERAS programme. The overall aim of this national meeting is to learn about the innovation and to increase motivation. The perioperative care elements and the effectiveness and safety of the ERAS programme will be discussed. Results of baseline measurement will be fed back and the gaps between actual practice and optimal practice will be demonstrated. The subject matters of this educational meeting are comparable to the kick-off meeting in the breakthrough series, but the number of participants is limited to a maximum of three per hospital team to ensure interactive participation, promote leadership, and limit costs. 


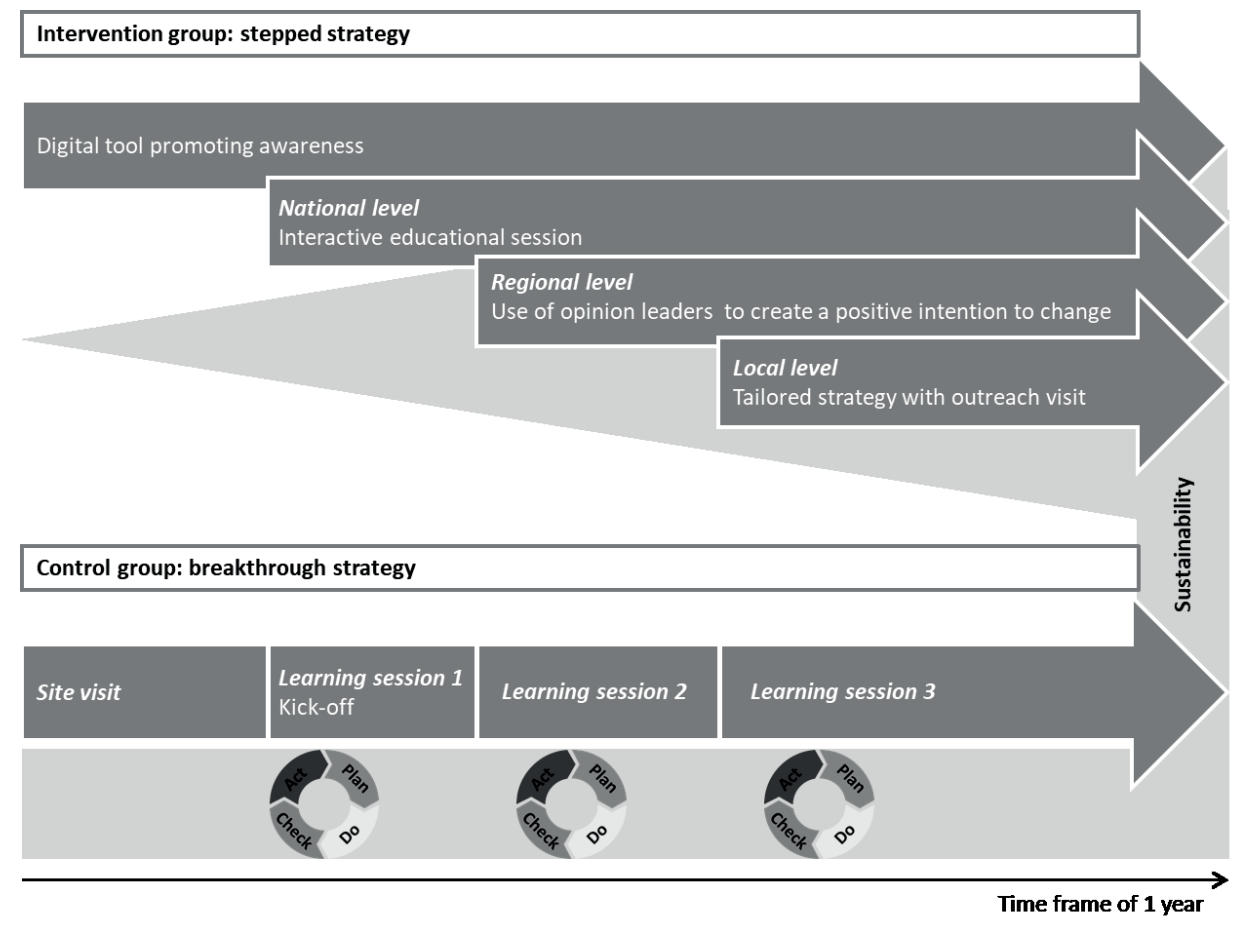

Figure 2. Overview of the stepped and breakthrough implementation strategy. The diagram shows the structure of the breakthrough strategy (control group) with standard iterative plan-do-study-act (PDSA) cycles and the stepped implementation strategy (intervention group) with four implementation levels depending on progress. The light grey color represents the intensity of external support

\section{Third implementation level}

The third step is, in contrast to the previous one, a regional activity and aims at influencing the views regarding the innovation and creating a positive intention to change. All locally involved professionals will be invited to participate to a regional meeting to learn from each other via exchange of experiences. Professional peers and opinion leaders will be used to convince them that the programme is valuable, effective, useful, and feasible.

\section{Fourth implementation level}

The fourth level is the most intensive one and acts on local level. Activities comprise identification of local barriers to change and selecting activities to promote the adoption of the programme. Members of the expert team will conduct outreach visits and will guide the local team through the process of tailoring interventions. Again, audit data will be used to define to what extent the team deviates from best practice. Local cultural and environmental issues that hinder or facilitate the change 
process will be identified and implementation activities will be carefully linked to these barriers and facilitators to change.

The decision to step up to a higher level of implementation activities will be based on the success of implementation. The standards of implementation success include the following: at least $80 \%$ of patients recovered within three days and at least $60 \%$ of patients going home within five days after surgery. Although we expect the hospitals to include about 25 patients per established evaluation period of three months, we assume that improvements need some time to get incorporated in routine practice. The final decision on the implementation level will be a shared decision with the hospital, which might have good reasons for clarifying the lack of success or for preferring to step up to a higher level of implementation intensity even though the benchmark of success was reached.

\section{Outcome measures}

Process and clinical outcome data will be measured by pre-defined indicators of success to study contextual factors that are critical to the change process. An effectiveness, economic, and process evaluation will be conducted throughout the trial.

\section{Effect evaluation}

The primary outcome measure is length of hospital stay, defined as the number of nights that a patient stayed in hospital after surgery. Secondary outcome measures are length of recovery and guideline adherence. Recovery is defined as the first day after surgery that the patient fulfilled all three discharge criteria: tolerance of food, adequate pain control on oral analgesics, and independency in activities or daily living. The complete ERAS programme, as proposed by the ERAS Society ${ }^{\circledR}$ [2], includes over twenty protocol care elements. Index elements were selected as having high level of evidence, being readily recordable or having a direct influence on recovery. Those elements are: pre-admission counselling, no bowel preparation, carbohydrate load, use of prophylactic antiemetics, removal of nasogastric tube on end of surgery, no peritoneal drainage, start fluids on day 0 , start normal food and mobilisation on day 1, use of epidural analgesia, use of laxatives, and urinary catheter removal on day 2. Guideline adherence to the index elements will be registered for all eligible patients during the study period.

\section{Economic evaluation and budget impact}

An economic evaluation will be performed. Research has shown that ERAS leads to cost savings without compromising patients' safety or quality of life [29-31]. As neither a difference in health outcomes are expected from implementation of ERAS in gynaecological surgery nor a difference in costs outside the hospital [32] 
(i.e. productivity costs), the economic evaluation will basically take the form of a cost-minimization analysis and will be performed from a hospital perspective. The effectiveness of the implementation strategies (length of postoperative hospital stay) can be expressed as costs. Therefore, for both strategies, implementation costs will be calculated and compared with the cost savings due to the reduction in hospital stay. The strategy which returns the lowest net costs is considered to be the most cost-effective.

\section{Process evaluation}

The quantitative results will be complemented with a process evaluation. Normally, the outcomes for process evaluation are only measured in the intervention arm. In this comparative effectiveness research study, we will also measure the uptake of the implementation strategy in the control arm. The evaluation will provide valuable insights in potential barriers and facilitators that affected the effectiveness of both implementation strategies. Process data will be collected in line with a framework provided by Hulscher et al. to address actual intervention exposure and to describe experiences with the intervention [33]. Implementation fidelity will be monitored by the registration of guideline adherence to the index protocol elements during all phases of perioperative care. The registration of the number and type of professionals involved and the attendance of the educational meetings will be measured. The barriers that emerge during the educational sessions and outreach visits and the solutions to overcome these barriers will be monitored as well. Besides, all communication between the expert and local teams will be registered.

\section{Data collection}

Before the start of implementation activities, a baseline audit of process and outcome data will be performed by reviewing medical charts of the first 30 patients treated in 2012. This period was chosen as the ERAS programme was not discussed yet in the DGOG. In case of an insufficient number of patients, the retrospective audit will be completed with patients operated in 2011. Hereafter, from the first day of the start till the end of the project 1 year later, a member of the expert team will monitor process and outcome data for both the intervention and the control group. An electronic database supporting good clinical practice guidelines will be available. Patient characteristics, process indicators, and outcome measures (proportion of patients reaching functional recovery within 3 days and proportion of patients hospitalised for a maximum of 5 days) will be audited for every 3-month period during the 1-year implementation period. The timing of the teaching sessions of the breakthrough project will be synchronously with the timing of implementation steps (Fig. 2). Although the effectiveness of both strategies will be evaluated and 
compared every 3 months after every step or teaching session, the main goal of the study is to compare effectiveness at the end of the project using all patients collected during the year. Costs for the two implementation strategies will be calculated from hours and tariffs for the strategy delivery team (CBO expert, subject matter experts) as well as all costs for surgeons, anaesthetists and nurses attending implementation related activities, including time, travel, and conference expenses. Furthermore, the costs of materials will be calculated. Additional costs of the audit database will be calculated as well, as this is an essential part of the implementation strategies. Standardised forms will be developed for the measurement of activities, materials and expenses associated with implementation [34]. Those forms will be filled in by members of the implementation team at each site and will provide information for the economic and process evaluation of the quality improvement. Qualitative data regarding the process evaluation will be derived from extensive notes of all contacts with each hospital and during the educational sessions. Minutes will be taken by at least two members of the expert or local implementation team.

\section{Sample size}

With the support of the DGOG we expect to include the majority of Dutch hospitals, and all patients that will be exposed to major gynaecological surgery during the implementation year. Approximately 100 patients undergo surgery per hospital each year. We do not expect drop outs among patients as informed consent procedures and filling in questionnaires by patients is not needed. The history of adherence to the ERAS protocol in the Netherlands during the implementation year will be described, so this study is in principle not about statistics and sample size calculations. However, it may be still worthwhile to calculate what statistical differences we will be able to assess, taking the perspective that this sample can be seen as an estimate for the future health care, or assuming that some hospitals will not participate as expected. The stepped implementation strategy is expected to be superior to the breakthrough strategy in improving perioperative care processes and reducing length of hospital stay. Anticipating a total adherence of $50 \%$ to the benchmark of length of stay of 5 days or less in the breakthrough hospitals versus $70 \%$ adherence in the stepped implementation hospitals, we would need to randomise seven hospitals per arm, each including at least 60 patients during the whole study period. Sample size computation was based on an estimated intra-class correlation coefficient (ICC) of 0.06 in secondary care, a power level of $80 \%$ and a significance level $(\alpha)$ of 0.05 . The intra-class correlation coefficient used to calculate the sample size was based on a study of Campbell et al. Although this study reported significant lower ICCs for outcome variables compared to process variables, we have conservatively computed the sample size using the highest applicable ICC of 0.06 for secondary care. Using an 
inflation factor of $11 \%$ to account for unequal cluster size yields a recruitment target of 67 patients per cluster. It is possible that no clinically relevant differences $(<20 \%)$ between arms could be found. In such case, we expect the stepped implementation programme still to be superior from a financial perspective.

\section{Data analysis \\ Effect evaluation}

Descriptive statistics (proportions, mean, and median) will be performed for process and outcome data sets. Differences between groups will be evaluated using independent- samples t-test for numerical variables and Chi-square test for categorical variables. Since patients are nested within hospitals, implementation effects will be analysed using linear or logistic mixed-effects model analysis for numerical or binary outcome measures, accounting for a random hospital effect. Since the four implementation periods can be considered as different (time) groups, this time variable will be included as a factor in the regression model. Besides, subgroup analyses will be performed to describe trends in implementation over time in more detail. Noninferiority comparisons will be presented for outcome measures using $95 \%$ confidence intervals. Two-sided p-values $\leq 0.05$ will be considered as statistically significant. Statistical analyses will be performed using SPSS ${ }^{\circledR}$ version 22 software (SPSS, Chicago, Illinois, USA).

\section{Economic evaluation and budget impact}

The uncertainty as regards the cost-minimisation results will be quantified using bootstrapping techniques. Subgroup and sensitivity analysis will be performed to test for the robustness of results. An effectiveness analysis will be performed every 3 months after completing a learning session. Thus, we will be able to judge to what level of intensity the activities are worth the effort. Implementation costs and effectiveness (savings due to reduction in hospital stay) will be calculated for each period, as well as cumulative implementation costs and cumulative effectiveness. A budget impact analysis will be performed addressing the financial stream of consequences related to the uptake and diffusion of the ERAS programme to assess affordability [36]. The budget impact will depend on both the costs of both strategies, the effect in terms of hospital stay reduction as well as the current level of uptake of ERAS.

\section{Process evaluation}

Quantitative process data of the implementation study will be analysed by means of descriptive statistics. Potential barriers and facilitators for successful implementation of the ERAS programme will be extracted, and open text data will be categorised 
in codes. Content analysis will be performed to analyse the obtained qualitative data of barriers and contextual factors of the implementation process.

\section{Ethical approval}

The Medical Ethics Committee of the University of Maastricht confirmed this quality assurance study (METC 13-5-031) was not subjected to the Dutch Clinical Research involving Human Subject Act (WMO). Therefore, informed consent at patient level was not necessary. Privacy of patients' information is protected by coding and processing all data anonymously.

\section{DISCUSSION}

This paper presents the protocol of a cluster randomised controlled study that takes up the challenge to design an efficient strategy in large scale implementation. Implementation of new evidence in practice is complex and evaluations have shown a modest to moderate impact of implementation processes on the achievement of results [37]. Limited resources increase the need for more efficiency during the implementation process. Therefore, it is important to explore if there are simpler and cheaper ways to achieve same outcomes. To our knowledge, this is the first study that used an innovative stepped strategy combining a tailored and successive implementation approach on national level. We lack a validated instrument to measure the stage of change and the need for more or less intensive implementation strategies. In the current study, process evaluation is an important tool to gain insight why and for whom the developed implementation strategy works. Reviews of implementation strategies show that interventions are successful in some settings, but fail in others [37, 38]. This stepped strategy allows us to study for whom and why pure diffusion is effective to change and for whom and why a surplus of an educational session, the use of opinion leaders, or a tailored strategy is required. Addressing the substantial gap between best practices on perioperative care and current care provided around major gynaecological surgery, the study focuses on large scale implementation of the Enhanced Recovery After Surgery (ERAS) programme in the Netherlands. Most Dutch hospitals authorised to perform major gynaecological surgery will participate in this study, providing representative results. The ERAS programme contains several elements to promote early recovery from major surgical trauma and consequently reducing length of hospitalisation. Key index elements were selected to ensure complete and correct recording of data. Besides, data collection was carried out by a member of the expert team, limiting bias in the data collection. ERAS programmes showed to be cost-saving [30, 39]. However, 
resistance to implement ERAS may arise due to competing priorities and pressure in the workplace. Those issues challenge adoption of ERAS in both implementation groups. Process analysis will help to explore perceived barriers and facilitators and will contribute to understand and explain study outcomes. This practice-based study follows an intention to treat analysis to prevent overestimation of the implementation effect. One of the limitations of the study is the short time to evaluate the effect of the different implementations steps. Although a progressive improvement in process and clinical outcomes might be expected, the 3-month evaluation periods were chosen to follow the timing of the breakthrough educational sessions. Comparing effectiveness and costs of a stepped implementation approach with tailoring intensity of interventions to the needs of organisations to the traditionally used breakthrough strategy will help to define a preferred strategy for nationwide dissemination of best practices. 


\section{REFERENCES}

1. Kehlet H. Multimodal approach to control postoperative pathophysiology and rehabilitation. Br J Anaesth. 1997;78(5):606-17.

2. Nygren J, Thacker J, Carli F, Fearon KC, Norderval S, Lobo DN, et al. Guidelines for perioperative care in elective rectal/pelvic surgery: Enhanced Recovery After Surgery (ERAS) Society recommendations. World J Surg. 2013;37(2):285-305.

3. Gatt M, Anderson AD, Reddy BS, Hayward-Sampson P, Tring IC, MacFie J. Randomized clinical trial of multimodal optimization of surgical care in patients undergoing major colonic resection. Br J Surg. 2005;92(11):1354-62.

4. Maessen J, Dejong CH, Hausel J, Nygren J, Lassen K, Andersen J, et al. A protocol is not enough to implement an enhanced recovery programme for colorectal resection. $\mathrm{Br} \mathrm{J}$ Surg. 2007;94(2):224-31.

5. Spanjersberg WR, Reurings J, Keus F, van Laarhoven CJ. Fast track surgery versus conventional recovery strategies for colorectal surgery. Cochrane Database Syst Rev. 2011;2:CD007635.

6. Varadhan KK, Neal KR, Dejong CH, Fearon KC, Ljungqvist O, Lobo DN. The Enhanced Recovery After Surgery (ERAS) pathway for patients undergoing major elective open colorectal surgery: a meta-analysis of randomized controlled trials. Clin Nutr. 2010;29(4):434-40.

7. Carter J, Szabo R, Sim WW, Pather S, Philp S, Nattress K, et al. Fast track surgery: a clinical audit. Aust N Z J Obstet Gynaecol. 2010;50(2):159-63.

8. Polle SW, Wind J, Fuhring JW, Hofland J, Gouma DJ, Bemelman WA. Implementation of a fast-track perioperative care program: what are the difficulties? Dig Surg. 2007;24(6):441-9.

9. Gravante G, Elmussareh M. Enhanced recovery for non-colorectal surgery. World J Gastroenterol. 2012;18(3):205-11.

10. Carter J. Fast-track surgery in gynaecology and gynaecologic oncology: a review of a rolling clinical audit. ISRN Surg. 2012;2012:368014.

11. de Groot JJ, van Es LE, Maessen JM, Dejong CH, Kruitwagen RF, Slangen BF. Diffusion of enhanced recovery principles in gynecologic oncology surgery: is active implementation still necessary? Gynecol Oncol. 2014;134(3):570-5.

12. Lassen K, Hannemann P, Ljungqvist O, Fearon K, Dejong CH, von Meyenfeldt MF, et al. Patterns in current perioperative practice: survey of colorectal surgeons in five northern European countries. BMJ. 2005;330(7505):1420-1.

13. Ansari D, Gianotti L, Schroder J, Andersson R. Fast-track surgery: procedure-specific aspects and future direction. Langenbecks Arch Surg. 2013;398(1):29-37.

14. Kehlet H, Wilmore DW. Evidence-based surgical care and the evolution of fast-track surgery. Ann Surg. 2008;248(2):189-98.

15. Carter J. Laparoscopy or laparotomy for early endometrial cancer? Lancet Oncol. 2010;11(11):1021-2. author reply 2-3.

16. Mourits MJ, Bijen CB, Arts HJ, ter Brugge HG, van der Sijde R, Paulsen L, et al. Safety of laparoscopy versus laparotomy in early-stage endometrial cancer: a randomised trial. Lancet Oncol. 2010;11(8):763-71. 
17. Institute for Healthcare Improvement. The Breakthrough Series: IHI's collaborative model for achieving breakthrough improvement. IHI Innovation Series white paper. Boston: Institute for Healthcare Improvement; 2003.

18. Kilo CM. A framework for collaborative improvement: lessons from the Institute for Healthcare Improvement's Breakthrough Series. Qual Manag Health Care. 1998;6(4):113.

19. Schouten LM, Hulscher ME, van Everdingen JJ, Huijsman R, Grol RP. Evidence for the impact of quality improvement collaboratives: systematic review. BMJ. 2008;336(7659):1491-4.

20. Schouten LM, Niessen LW, van de Pas JW, Grol RP, Hulscher ME. Costeffectiveness of a quality improvement collaborative focusing on patients with diabetes. Med Care. 2010;48(10):884-91.

21. Øvretveit J, Klazinga N. Meta-evaluation of the ten national quality improvement programs in The Netherlands 2004-2009. The Hague, the Netherlands: The Netherlands Organization for Health Research and Development (ZonMw); 10:106 Page 8 of 9

22. Grol R, Wensing M. What drives change? Barriers to and incentives for achieving evidence-based practice. Med J Aust. 2004;180(6 Suppl):S57-60.

23. Grol RP, Bosch MC, Hulscher ME, Eccles MP, Wensing M. Planning and studying improvement in patient care: the use of theoretical perspectives. Milbank Q. 2007;85(1):93-138.

24. Baker R, Camosso-Stefinovic J, Gillies C, Shaw EJ, Cheater F, Flottorp S, et al. Tailored interventions to overcome identified barriers to change: effects on professional practice and health care outcomes. Cochrane Database Syst Rev. 2010;3:CD005470.

25. Wensing M, Oxman A, Baker R, Godycki-Cwirko M, Flottorp S, Szecsenyi J, et al. Tailored Implementation For Chronic Diseases (TICD): a project protocol. Implement Sci. 2012;6:103.

26. Langley G, Nolan K, Nolan T, Norma C, Provost L. The improvement guide: a practical approach to enhancing organisational performance. San Francisco: Jossey-Bass Publishers; 1996.

27. de Kok M, van der Weijden T, Voogd AC, Dirksen CD, van de Velde CJ, Roukema JA, et al. Implementation of a short-stay programme after breast cancer surgery. Br J Surg. 2010;97(2):189-94.

28. Gillissen F, Hoff C, Maessen JM, Winkens B, Teeuwen JH, von Meyenfeldt MF, et al. Structured synchronous implementation of an enhanced recovery program in elective colonic surgery in 33 hospitals in The Netherlands. World J Surg. 2013;37(5):1082-93.

29. Khan S, Wilson T, Ahmed J, Owais A, MacFie J. Quality of life and patient satisfaction with enhanced recovery protocols. Colorectal Dis. 2010;12(12):1175-82.

30. Ghosh K, Downs LS, Padilla LA, Murray KP, Twiggs LB, Letourneau CM, et al. The implementation of critical pathways in gynecologic oncology in a managed care setting: a cost analysis. Gynecol Oncol. 2001;83(2):378-82.

31. Morris M, Levenback C, Burke TW, Dejesus Y, Lucas KR, Gershenson DM. An outcomes management program in gynecologic oncology. Obstet Gynecol. 1997;89(4):485-92.

32. Hjort Jakobsen D, Sonne E, Basse L, Bisgaard T, Kehlet H. Convalescence after colonic resection with fast-track versus conventional care. Scand J Surg. 2004;93(1):24-8.

33. Hulscher ME, Laurant MG, Grol RP. Process evaluation on quality improvement interventions. Qual Saf Health Care. 2003;12:40-6. 34. de Kok M, Dirksen CD, Kessels AG, 
van der Weijden T, van de Velde CJ, Roukema JA, et al. Cost-effectiveness of a short stay admission programme for breast cancer surgery. Acta Oncol. 2010;49(3):338-46.

35. Campbell MK, Fayers PM, Grimshaw JM. Determinants of the intracluster correlation coefficient in cluster randomized trials: the case of implementation research. Clin Trials. 2005;2(2):99-107.

36. Mauskopf JA, Sullivan SD, Annemans L, Caro J, Mullins CD, Nuijten M, et al. Principles of good practice for budget impact analysis: report of the ISPOR Task Force on good research practices-budget impact analysis. Value Health. 2007;10(5):336-47.

37. Grimshaw JM, Thomas RE, MacLennan G, Fraser C, Ramsay CR, Vale L, et al. Effectiveness and efficiency of guideline dissemination and implementation strategies. Health Technol Assess. 2004;8(6):iii-iv. 1-72.

38. Grol R. Personal paper. Beliefs and evidence in changing clinical practice. BMJ. 1997;315(7105):418-21. 39. Lee J, Tollefson E, Daly M, Kielb E. A generalized health economic and outcomes research model for the evaluation of companion diagnostics and targeted therapies. Expert Rev Pharmacoecon Outcomes Res. 2013;13(3):361-70.

39. Lee J, Tollefson E, Daly M, Kielb E. A generalized health economic and outcomes research model for the evaluation of companion diagnostics and targeted therapies. Expert Rev Pharmacoecon Outcomes Res. 2013;13(3):361-70. 



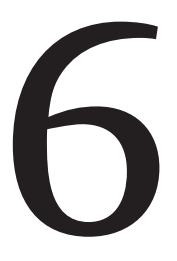

Evaluating a tailored stepped implementation strategy on the improvement of perioperative care in gynecologic oncology A cluster randomized clinical trial

\author{
Jeanny J.A. de Groot \\ Brigitte F.M. Slangen \\ José M.C. Maessen \\ Bjorn Winkens \\ Carmen D. Dirksen \\ Trudy van der Weijden
}

on behalf of all the members of the study group 


\section{ABSTRACT}

Importance Implementation of an enhanced recovery after surgery program to improve perioperative care on large scale in an effective manner is challenging. The optimal strategy for a multicenter quality improvement program remains undetermined.

Objective To evaluate the nationwide implementation of an enhanced recovery after surgery program in gynecologic surgery by using two strategies: a traditional breakthrough strategy and a tailored stepped implementation design.

Design Cluster randomized controlled trial.

Setting and participants Fourteen hospital gynecology units in The Netherlands performing major abdominal surgery in gynecologic oncology patients. A total of 1048 patients underwent surgery during the one-year implementation period (June 2014 to June 2015). Baseline data were retrospectively collected.

Interventions The intervention group (7 hospitals, 456 patients) received a tailored stepped implementation strategy comprising four levels of intensity of support. The decision for a stepwise increase in intensive support was hospital based. In the control arm (7 hospitals, 642 patients), hospitals received the traditional breakthrough strategy with generic educational sessions and the use of plan-do-study-act cycles for planning and executing local improvement activities.

Main outcomes and measures The length of postoperative hospital stay (primary outcome) and the length of recovery, and guideline adherence (secondary outcomes) were measured during a one-year implementation period. An effect and process evaluation was conducted.

Results Mean length of hospital stay was $5.8 \pm 3.9$ days in the breakthrough and $5.8 \pm 3.9$ days in the tailored stepped implementation group (MD 0.03, 95\% CI -0.44 to $0.50, \mathrm{p}=0.905)$. Both strategies significantly improved perioperative care, with a guideline adherence of $77.2 \pm 1.0 \%$ and $74.0 \pm 1.5 \%$, respectively (MD 1.7, 95\% CI -0.2 to $6.8, \mathrm{p}=0.058$ ) during the last implementation phase. Mixed-effect regression models did not reveal any differences in effect between the breakthrough and tailored stepped implementation strategy in postoperative length of hospital stay (mean difference (MD) -0.5 days; $95 \% \mathrm{CI}-1.9$ to $0.9 ; \mathrm{p}=0.476$ ), length of recovery (MD -0.36 days; $95 \%$ CI -1.47 to $0.76 ; \mathrm{p}=0.497$ ); and overall guideline adherence (MD -3.0\%; 95\% 
CI -13.6 to 7.6; $\mathrm{p}=0.583$ ). The proportion of patients recovered within postoperative day 3 and discharged within postoperative day 5 was similar in the tailored stepped implementation group compared to the breakthrough implementation group (odds ratio 1.56 ; $95 \%$ CI 0.79 to $3.07 ; \mathrm{p}=0.203$ and odds ratio $1.40 ; 95 \%$ CI 0.50 to 3.92 ; $\mathrm{p}=0.527$, respectively). Sensitivity and subgroup analysis showed similar results. Process data showed poor fidelity in the tailored stepped implementation group.

Conclusions and relevance Both implementation strategies improved perioperative care and outcomes. The tailored stepped implementation strategy did not differ in effectiveness compared to the breakthrough strategy for health care improvement on large scale, but process analysis showed a poor fidelity to implementation activities. The study underlines the importance of evaluating both effect outcomes and process measures of innovative strategies. 


\section{BACKGROUND}

Enhanced recovery after surgery (ERAS) programs are widely accepted as a new standard in patient care [1]. The multimodal program combines several core elements, in particular relating to pain, nutritional and mobilization management [2]. Over time, those programs have been implemented in several surgical settings to optimize perioperative care, accelerate recovery and reduce morbidity after surgery. Their effect has been studied on both local and large-scale level and has already proved to represent efficient practice [1,3]. Although perioperative care among different fields of surgery has improved, for gynecological patients perioperative care has not changed substantially yet and adoption of ERAS programs within and across hospitals remains a challenge.

Despite positive research findings, best clinical practices are not widely used and further knowledge on how to disseminate and implement the ERAS program is necessary. Most former research efforts have focused on the effectiveness of the ERAS program to improve quality of perioperative care in various settings by using an implementation strategy [4]. The current evidence lacks the focus on what type of implementation strategy can best be used to achieve this quality improvement.

The so-called 'breakthrough' implementation strategy has proven to be an effective strategy for implementation of healthcare innovations across different organizations [5, 6]. Originally, the components of the 'breakthrough' strategy were captured in a Breakthrough Series model developed by the Institute of Healthcare Improvement [7, 8]. This multifaceted strategy uses an underlying framework of collaborative learning sessions and action periods (Plan-Do-Study-Act cycles) to apply short-term implementation interventions in order to improve healthcare outcomes on a large scale $[7,8]$. Implementation interventions can be defined as techniques designed to enhance adoption of a specific clinical practice to improve health care outcomes and are part of an implementation strategy [9]. Examples are distribution of educational materials, educational meetings, and audit and feedback [10].

The Breakthrough Series model and its variants are the most widely used evidence-based quality improvement collaborative [5, 6]. Reviews include mainly observational data of pre-post and interrupted time series studies [5, 6, 11]. Data have shown that, besides the moderately positive effects observed, the evidence of the actual impact of a specific quality improvement strategy on health outcomes is limited [5, 6]. Implementation of quality improvement initiatives on large scale remains suboptimal and evidence about their impact could be improved. Part of this complexity owes to the multiple components and different settings involved, the required resources, and the empowerment of different health care professionals needed to successfully adjust clinical practice across different healthcare settings. Research on how to optimize resources is warranted [6]. 
Adapting current implementation strategies to a more efficient, tailored model is important for effective large scale implementation and was studied in the present study. The aim of this study was to evaluate the strategy used by implementing ERAS across multiple hospitals in order to define a preferred strategy for the large scale implementation of evidence-based practice. A hybrid-effectiveness implementation design was used [9], by comparing the breakthrough implementation strategy with a new tailored stepped approach designed to deliver an optimal effect of implementation interventions with minimal investments, and to identify intervention fidelity and contextual factors that influence implementation by means of a process evaluation.

\section{METHODS}

\section{Study design}

A multicenter, cluster randomized controlled trial was conducted to compare the effect of two different implementation strategies to support uptake of an ERAS program in multiple hospitals while gathering information about related clinical outcomes. Hospitals were randomly allocated by an independent statistician using cluster randomization, stratified by the type of hospital. Stratification was used to prevent unbalanced study groups with respect to surgical complexity of high-volume tertiary hospitals and their affiliated regional hospitals. Hospitals in both study arms underwent a retrospective measurement of 30 patients to assess baseline perioperative practice and received an implementation strategy consisting of a variety of implementation interventions. Approval and a waiver from the need to provide informed consent were obtained from the Maastricht Ethical Committee (METC 13-05-031). Institutional review boards of study hospitals individually approved participation. The study protocol was registered in the Dutch Trial Register for clinical trials (record number NTR4058) and has been published previously in more detail [12].

\section{Clinical intervention}

This study focused on the nationwide implementation of the ERAS program. This perioperative management program was developed by the ERAS Society ${ }^{\circledR}$ to enhance recovery from major surgical trauma and to reduce postoperative morbidity [2]. A standardization of health care is realized by combining several evidence based care elements during the pre-, intra- and postoperative phase. The guideline for perioperative care as proposed by the ERAS Society ${ }^{\circledR}$ was used as a template for quality improvement [2]. 


\section{Implementation strategies}

Two different implementation strategies, the breakthrough strategy and a tailored stepped strategy, were compared to explore which strategy could be preferred to facilitate efficient large-scale implementation of a quality improvement initiative. Key features of both strategies are described in the study protocol [12]. To support the nationwide implementation of ERAS, all participating hospitals agreed to actively implement ERAS in order to improve their perioperative care. Both implementation strategies started at the same time and timing of the implementation steps was synchronized between both comparative strategies.

\section{Breakthrough implementation strategy}

The breakthrough implementation strategy was performed in a traditional way with general learning sessions based on feedback within the elaborative PDSA cycle, and could be regarded as the control group. The strategy is based on an underlying framework of a site visit followed by three collaborative learning sessions with a three month interval. These learning sessions were chaired by an agent of the Dutch Institute for Healthcare Improvements (CBO). An expert panel was available throughout the implementation process. This panel consisted of subject matter experts and an implementation expert. All participating hospitals were instructed to appoint a multidisciplinary team to lead the local improvement. Teams were expected to participate in all learning sessions.

\section{Tailored stepped implementation strategy}

An innovative tailored stepped implementation strategy was designed based on a combination of theoretical backgrounds and experiences of prior implementation projects [12]. The strategy aims to optimize resources by tailoring implementation efforts to specific needs of an organization. Each step leads to a more intensive stage in the process of change. Implementation started with generic low-intensive support including a digital toolbox (step 1), followed by a local interactive learning session (step 2), a regional peer review session (step 3), and might build up to the highest level of tailored and labor-intensive outreach visit (step 4), if necessary. The decision to step up to a higher level of implementation activities was evaluated every three months and participation depended on the success of implementation so far [12]. Three-monthly audit and feedback data were provided for all hospitals throughout the implementation process.

\section{Setting}

All tertiary referral hospitals and their affiliated regional hospitals authorized to perform major abdominal surgery in gynecologic oncology patients in the Nether- 
lands were eligible for participation. This authorization is based on a volume norm set by the Dutch Association of Gynecologists with a minimum of 20 cytoreductive surgeries for ovarian cancer per hospital each year. Besides, these cytoreductive surgeries, other open procedures for suspected or diagnosed ovarian, uterine, or cervical cancer were included in the definition of major abdominal surgery.

In the Netherlands, 27 hospitals were eligible for participation at the start of the inclusion period in 2013. The hospitals that predominantly admitted patients to an external hospital replacement care facility after discharge were excluded to avoid interference in outcome measures. Eligible hospitals were informed about the study protocol and were recruited for participation in the trial. Specific details about the recruitment process were published earlier [13]. After permission to participate had been obtained, hospitals underwent a baseline audit to determine local perioperative management. Hospitals that already implemented the ERAS program successfully were excluded afterwards. Enrollment occurred between May 2013 and May 2014. The baseline audit was performed in 19 of the 27 hospitals authorized to perform major abdominal surgery in gynecologic oncology patients in the Netherlands. Data of this audit were published earlier and showed overall a median time to recovery of 4.0 (range 3.0 to 5.0) days and median length of hospital stay of 6.0 (range 4.0 to 10.5) days. For the randomized hospitals data are available in a supplementary file (Table S1).

Both implementation strategies were focused on a professional level, directed to individual hospitals and their key target groups. These key target groups included health professionals (gynecologists, anesthetists and nurses) involved during the perioperative process. No specific exclusion criteria were applied at patient level. All women, aged over 18 years, who underwent elective major abdominal surgery during the implementation period from June 2014 to June 2015, were included.

\section{Outcomes}

\section{Effect evaluation}

Predefined indicators were used to measure the successful implementation of ERAS. Length of hospital stay, defined as the number of nights that a patient stayed in the hospital after surgery, was determined as the primary outcome for this study. Secondary outcomes consisted of the length of recovery and guideline adherence. Recovery was reached when patients tolerated food, had adequate pain control on oral analgesics, and were independent in activities and daily living. A cut-off level for the length of recovery and length of hospital stay was determined and set on a recovery within three days and discharge within five days after surgery. Implementation success was defined as at least $80 \%$ of patients having recovered within three days and at least $60 \%$ of patients going home within five days after surgery. A composite 
score, including a selection of twelve yes/no index elements, was used to determine guideline adherence (0-100\%). Index elements included preadmission counseling, no bowel preparation, carbohydrate load, use of prophylactic anti-emetics, removal of nasogastric tube on end of surgery, no peritoneal drainage, start fluids on day 0 , start normal food and mobilization on day 1, use of epidural analgesia, use of laxatives, and urinary catheter removal on day 2 .

\section{Process evaluation}

To evaluate actual exposure and to describe experiences with both implementation strategies a prospective process evaluation was conducted in line with a framework described by Hulscher et al. [14]. Guideline adherence to specific index protocol elements, self-reports of implementation activities, and attendance registrars were used to monitor implementation fidelity. Barriers and facilitators that emerged during the implementation process were registered.

\section{Data collection}

Team and hospital characteristics were collected at the start of the study. Patient characteristics, process indicators, and outcome data were obtained prospectively by a member of the expert panel during the one-year implementation strategy. Data were entered in an electronic database designed according to the principles of good clinical practice research guidelines and were audited for every three-month period. Besides, extensive field notes were taken of each contact with local hospitals, minutes were taken during implementation activities, and quarterly time logs were collected after each implementation phase. Standardized forms with open text blocks to register implementation activities were available for the strategy delivery team and members of the local teams at each hospital. These data were used for the qualitative process evaluation. The retrospective baseline audit included a consecutive sample of 30 patients treated in 2012.

\section{Power calculation}

A total of seven hospitals per arm, each including at least 60 patients, were required to detect a $20 \%$ difference between the two study arms in the primary outcome (proportion of patients with a length of stay of 5 days or less). Sample size computation was based on an estimated intra-class correlation coefficient of 0.06 , a power level of $80 \%$ and a significance level of 0.05 . Using an inflation factor of $11 \%$ to account for unequal cluster size yields a recruitment target of 67 patients per cluster. Therefore, at least 938 patients were needed to enroll in the study. 


\section{Data analysis}

An intention to treat analysis was used for all outcomes. Descriptive statistics were used to describe process and outcome data sets. Since patients were nested within hospitals, implementation effects were analyzed using linear or logistic mixedeffect regression models for continuous or binary outcome measures, accounting for a random hospital effect. The four implementation phases (at 3, 6, 9 and 12 months) can be considered as different (time) groups; therefore this time variable was included as a factor in the regression model. Subgroup analyses were performed to describe trends in implementation over time in more detail. For some index elements adherence data contained missing values (supplementary table). Therefore, multiple imputation, with a maximum number of iterations set equal to 20 , for the missing values were used and 50 imputated datasets were created. Mixed regression analyses were applied to each dataset and then pooled. A p value $<0.05$ was considered to be statistically significant. Statistical analyses were performed using SPSS ${ }^{\circledR}$ software. Content analysis was used to analyze the qualitative data obtained with field notes, minutes and standardized forms. Evaluation criteria of the theory-based Consolidated Framework for Implementation Research (CFIR) were followed [15]. The CFIR captures five main domains (intervention characteristics, outer setting, inner setting, characteristics of individuals and process) associated with effective implementation. This evidence-based framework was used to systematically assess the data extraction process and to categorize the barriers and facilitators experienced during the implementation process.

\section{RESULTS}

Gynecology departments of 15 hospitals were eligible for participation and were cluster-randomized to the tailored stepped $(n=7)$ or breakthrough implementation group $(\mathrm{n}=8)$. One hospital had to withdraw directly after randomization because of loss of authorization to perform major abdominal surgery and therefore not fulfilling the inclusion criteria any more. Six Dutch tertiary referral hospitals and eight affiliated regional hospitals were included in the study and implemented ERAS during the one-year study period. Due to the intention-to-treat design, none of the hospitals discontinued the intervention (figure 1). In total, 1098 patients underwent surgery during the implementation process and were enrolled in the study (supplementary file, S2). Patients were linked to their hospital and their characteristics and surgical details were presented in table 1 for both the breakthrough $(\mathrm{N}=642)$ and the tailored stepped implementation group ( $\mathrm{N}=456)$. Although stratification on the type of hospital was performed, resulting in balanced groups at hospital level, a 
significant difference in the ratio of patients undergoing surgery in the high-volume tertiary care hospitals on patient level was found between the breakthrough and tailored stepped implementation group (69.9\% versus $61.8 \%$ respectively, $\mathrm{p}=0.005$ ). This resulted in more complex tertiary care procedures (for patients with ovarian and cervical cancer) in the breakthrough implementation group. Thirty-day mortality rate $(\mathrm{n}=3,0.5 \%$ versus $\mathrm{n}=3,0.6 \%)$ and readmission rate $(\mathrm{n}=20,5.5 \%$ versus $\mathrm{n}=20,4.4 \%)$ were comparable between the breakthrough and tailored stepped implementation study groups.

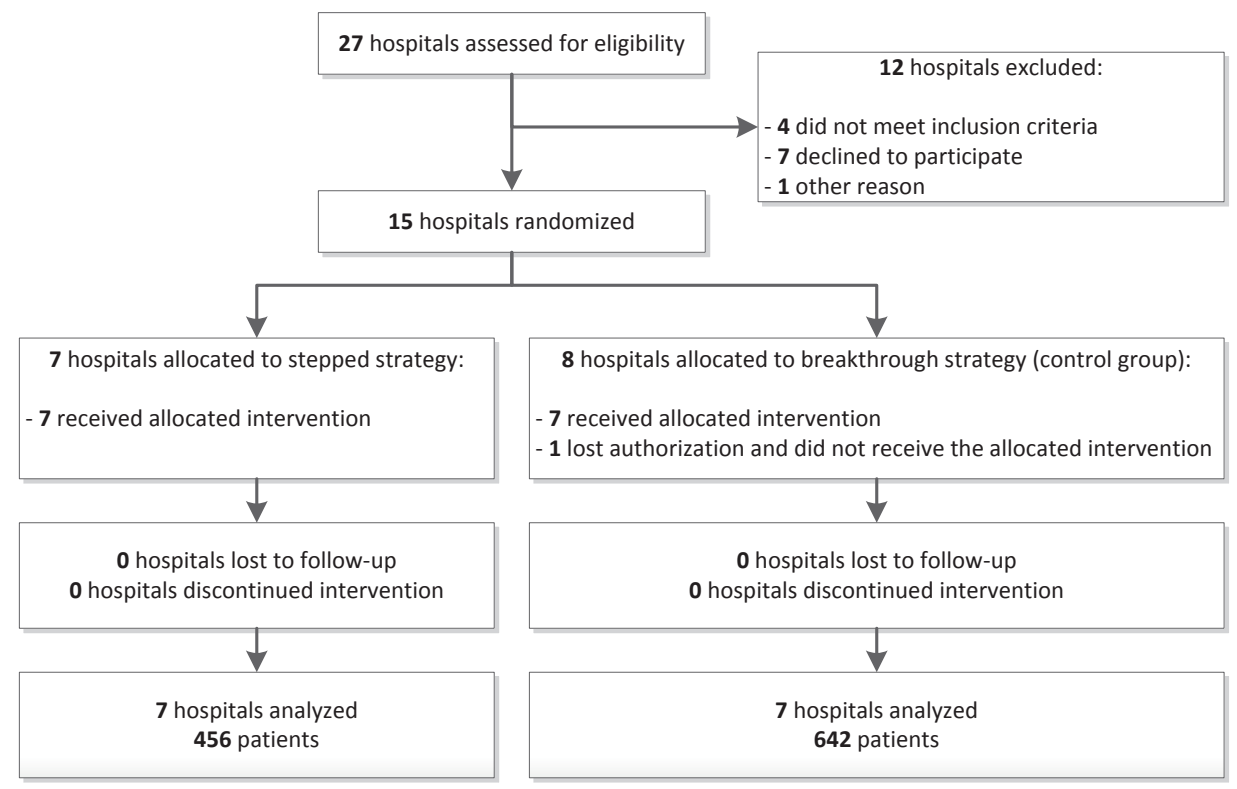

Figure 1 Flow chart

\section{Effect evaluation}

Mean length of hospital stay overall was $5.8 \pm 3.9$ days in the breakthrough and $5.8 \pm 3.9$ days in the tailored stepped implementation group (MD 0.03, 95\% CI - 0.44 to $0.50, \mathrm{p}=0.905$ ). Among hospitals undergoing the breakthrough strategy, ERAS implementation was associated with a significant improvement in recovery and admission times compared to data of the baseline audit (supplementary file, S1). Both implementation strategies significantly improved adoption of perioperative care practices with overall adherence rates of $67.5 \pm 16.9 \%$ in the breakthrough and $65.6 \pm 18.3 \%$ in the tailored stepped hospitals (supplementary file, S3 and S4). Figure 2 presents an overview of the guideline adherence to the selected twelve index elements (supplementary file, S5). During the last implementation period at 12 months (phase 4) composite scores of guideline adherence were comparable between the 
Table 1. Observed baseline characteristics and surgical details of patients by study group $(\mathrm{n}=1098)$.

\begin{tabular}{|c|c|c|}
\hline & Breakthrough & Stepped \\
\hline No. of patients & 642 & 456 \\
\hline Age in years, mean $\pm S D$ & $58.9 \pm 13.3$ & $61.6 \pm 12.69$ \\
\hline \multicolumn{3}{|l|}{ ASA classification, $\mathrm{n}(\%)$} \\
\hline $\mathrm{I} / \mathrm{II}$ & $577(89.9)$ & $400(87.7)$ \\
\hline III/IV & $65(10.2)$ & $56(12.3)$ \\
\hline \multicolumn{3}{|l|}{ (Suspected) diagnosis, n (\%) } \\
\hline Ovarian cancer & $421(65.6)$ & $329(72.1)$ \\
\hline Uterine cancer & $106(16.5)$ & $99(21.7)$ \\
\hline Cervical cancer & $115(17.9)$ & $28(6.1)$ \\
\hline \multicolumn{3}{|l|}{ Histological subtype, n (\%) } \\
\hline Benign & $114(17.8)$ & $89(19.5)$ \\
\hline Borderline / hyperplasia / dysplasia & $48(7.4)$ & $34(7.4)$ \\
\hline Malignant & $480(74.8)$ & $333(73.0)$ \\
\hline FIGO I & $189(39.4)$ & $396(28.8)$ \\
\hline FIGO II & $40(8.3)$ & $25(7.5)$ \\
\hline FIGO III & $151(31.5)$ & $117(35.1)$ \\
\hline FIGO IV & $52(10.8)$ & $51(15.3)$ \\
\hline Recurrence / residual tumor & $35(7.3)$ & $31(9.3)$ \\
\hline Metastases (other primary tumor) & $13(2.7)$ & $11(3.3)$ \\
\hline Unknown (no staging) & - & $2(0.6)$ \\
\hline \multicolumn{3}{|l|}{ Surgical approach, n (\%) } \\
\hline Midline incision & $633(98.6)$ & $431(94.5)$ \\
\hline Transverse incision & $9(1.4)$ & $25(5.5)$ \\
\hline Operation time in minutes, mean $\pm S D$ & $160.4 \pm 74.2$ & $148.3 \pm 84.3$ \\
\hline \multicolumn{3}{|l|}{ Type of surgery (only ovarian included), n (\%) } \\
\hline Explorative & $166(39.4)$ & $135(41.0)$ \\
\hline Staging (with/without lymphadenectomy) & $35(8.3)$ & $25(7.6)$ \\
\hline Primary cytoreductive & $100(23.8)$ & $71(21.6)$ \\
\hline Secondary cytoreductive & $120(28.5)$ & $98(29.8)$ \\
\hline \multicolumn{3}{|l|}{ Additional surgical procedure, n (\%) } \\
\hline Open-close (inoperable) & $15(2.3)$ & $17(3.7)$ \\
\hline Anastomosis & $43(6.7)$ & $26(5.7)$ \\
\hline Diverting stoma & $8(1.2)$ & $14(3.1)$ \\
\hline Lymphadenectomy & $222(34.6)$ & $103(22.6)$ \\
\hline Extensive upper abdominal surgery* & $36(5.6)$ & $43(9.4)$ \\
\hline
\end{tabular}

ASA = American Society of Anesthesiologists, FIGO = International Federation of Gynecology and Obstetrics, $\mathrm{SD}=$ standard deviation.

* Includes diaphragmatic stripping, partial liver resection, splenectomy, distal pancreatectomy, or cholecystectomy. 


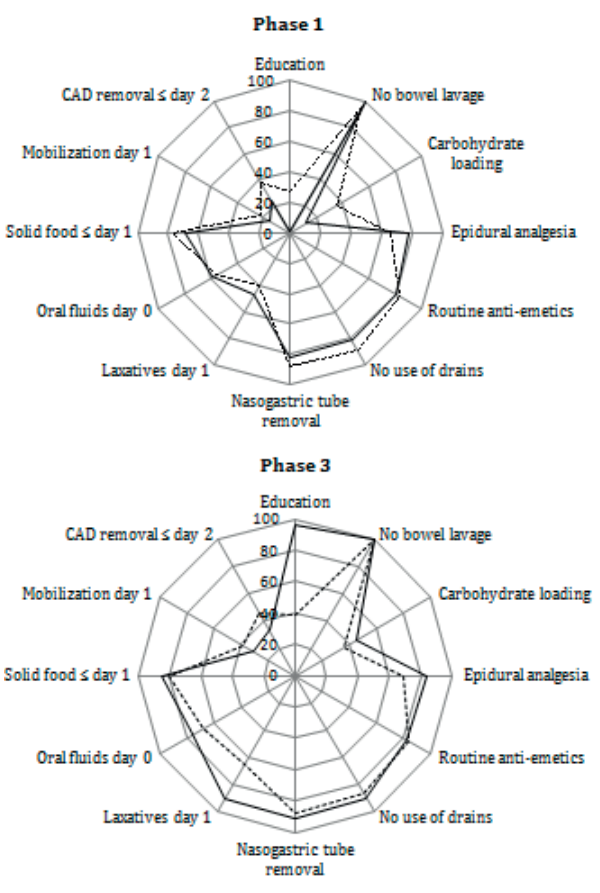

Figure 2 Overview of guideline adherence
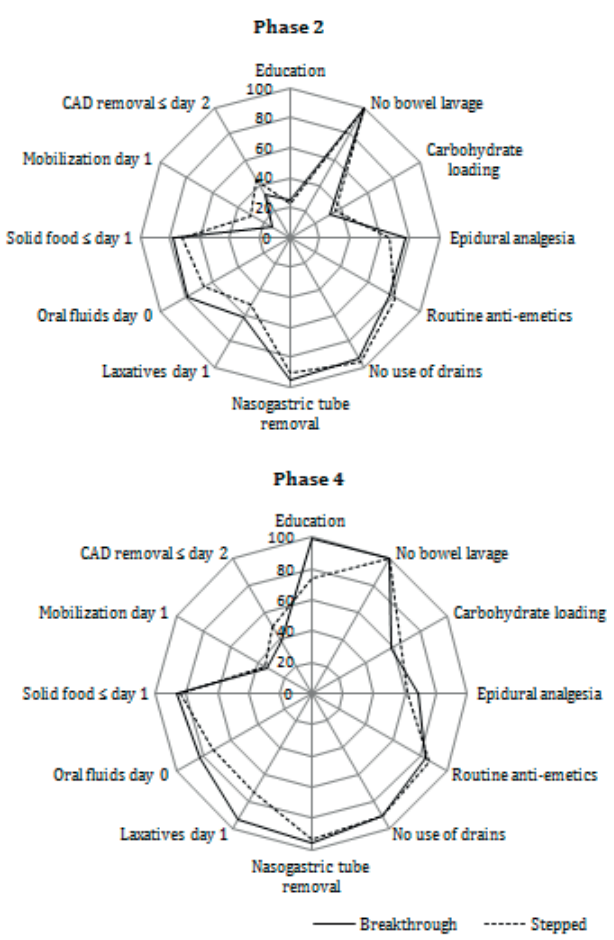

breakthrough and tailored stepped implementation group, $77.2 \pm 1.0 \%$ versus 74.0 $\pm 1.5 \%$, respectively ( $\mathrm{MD} 1.7,95 \% \mathrm{CI}-0.2$ to $6.8, \mathrm{p}=0.058$ ). At the 3 -month time point, five out of 12 elements had an adherence rate below $50 \%$. The five elements were similar between both study groups and included preadmission counseling, carbohydrate load, use of laxatives, mobilization on postoperative day 1 , and urinary catheter removal on day 2. The adherence to two protocol elements (mobilization and urinary catheter removal) remained below $50 \%$ at the 12 -month time point in both study groups.

Regression analysis of the type of implementation strategy, using random effects for clustering, found a positive but non-significant effect of the tailored stepped strategy versus the breakthrough strategy. The mean difference in effect during the implementation period on postoperative length of hospital stay was -0.5 days (95\% CI -1.9 to $0.9, \mathrm{p}=0.476$ ). The effect on secondary outcomes was also comparable between groups. The mean difference on length of functional recovery was -0.4 days (95\% CI -1.5 to $0.8, p=0.497)$ and on overall guideline adherence was $-3.0 \%$ (95\% CI -13.6 to $7.6, \mathrm{p}=0.583$ ). Table 2 shows that the proportion of patients recovered within postoperative day 3 and discharged within postoperative day 5 were similar 
Table 2. Regression analysis of the type of implementation strategy using random effects for clustering.

\begin{tabular}{|c|c|c|c|c|}
\hline & Breakthrough & Stepped & & \\
\hline & Mean $\pm S D$ & Mean $\pm S D$ & Adjusted MD (95\% CI) & P Value \\
\hline Protocol compliance (\%) & $67.5 \pm 16.9$ & $65.6 \pm 18.3$ & $-3.0(-13.6$ to 7.6$)$ & 0.583 \\
\hline Length of recovery (days) & $4.3 \pm 2.7$ & $4.3 \pm 2.4$ & $-0.4(-1.5$ to 0.8$)$ & 0.497 \\
\hline \multirow[t]{2}{*}{ Length of stay (days) } & $5.8 \pm 3.9$ & $5.8 \pm 3.9$ & $-0.5(-1.9$ to 0.9$)$ & 0.476 \\
\hline & $N(\%)$ & $N(\%)$ & Adjusted OR (95\% CI) & P Value \\
\hline$\%$ recovered $\leq 3$ days & $253 / 642(39.4)$ & $193 / 456(42.3)$ & $1.6(0.8$ to 3.1$)$ & 0.203 \\
\hline$\%$ discharged $\leq 5$ days & $407 / 642(63.4)$ & $277 / 456(60.7)$ & $1.4(0.5$ to 3.9$)$ & 0.527 \\
\hline
\end{tabular}

$\mathrm{SD}=$ standard deviation, $\mathrm{OR}=$ odds ratio.

in the stepped implementation group compared to the breakthrough implementation group.

To observe differences in the effect of the implementation process between both study groups sensitivity analysis were performed. Adding a time variable controlling for the different implementation phases did not substantially alter outcome measures. Corrected for their baseline performance (outcomes presented in supplementary file), subgroup analysis showed similar results. The odds ratios and 95\% confidence intervals are presented in figure 3 . These analyses showed a trend that differences in effect on recovery time were the largest during the first and second implementation phase. This trend was not observed in the effect on time to discharge.

\section{Process evaluation}

Local implementation teams varied in size and membership. The median number of members in the breakthrough strategy group was five persons (range, 2-9) compared to three in the stepped strategy group (range, 2-6). All teams included gynecologists and (specialized) nurses (practitioners). Besides, some teams included anesthesiologists, specialist registrars, quality assurance professionals, unit managers, outpatient clinical nurses, research nurses, physician assistants or dieticians.

\section{Breakthrough implementation strategy}

During the first implementation phase, hospitals randomized to the breakthrough implementation strategy were visited by two members of the expert panel to clarify the collaborative process and implementation goals. The first learning session (kickoff meeting) focused as intended on the gap between ERAS elements and current care, and was attended well, by 19 professionals (range, 2 to 5 per hospital). The second and third educational session were attended by 18 (range, 1 to 5 per hospital) 


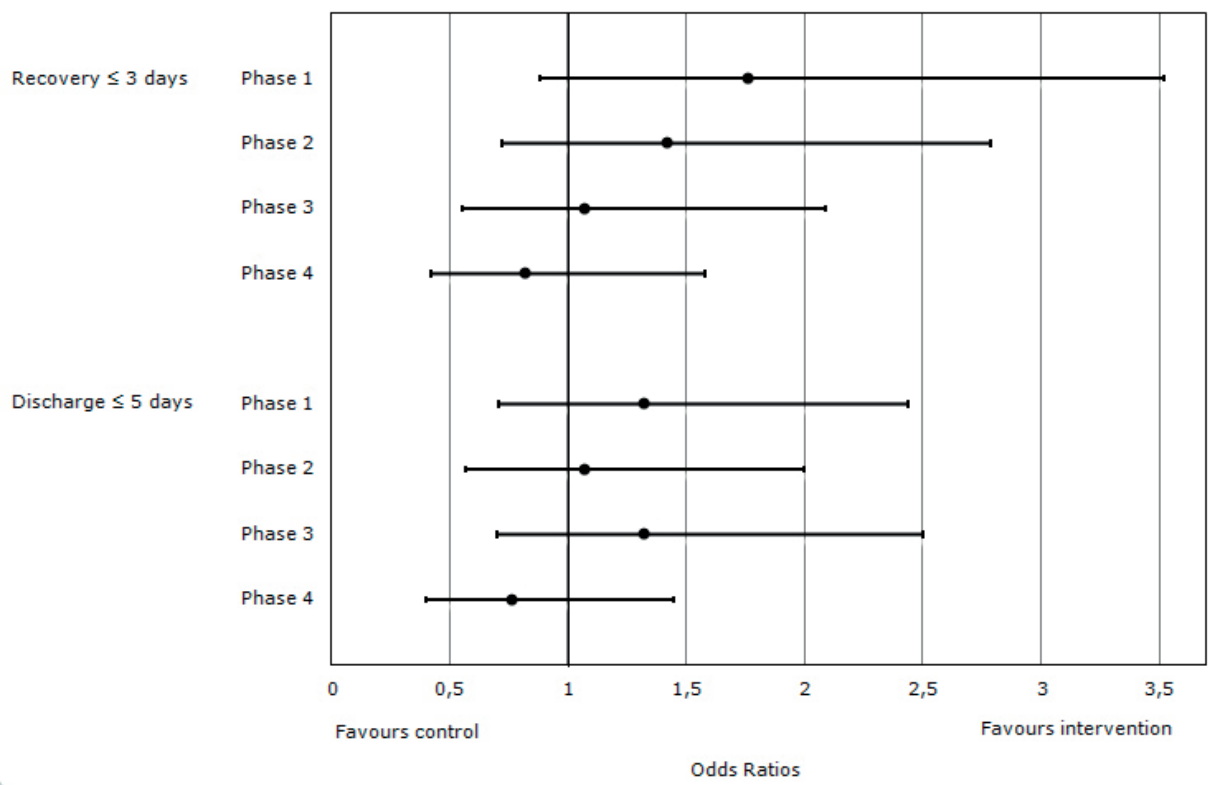

Figure 3 Odds ratios and 95\% confidence intervals, adjusted for clustering and baseline performance. Each point represents a 3-month period.

and 17 (range, 1 to 4 per hospital) professionals, respectively. Overall, mainly (specialized) nurses attended the meetings (66.7\%).

During the educational sessions, hospitals exchanged their experiences and reported their successes and barriers. Content analysis following the CFIR framework revealed that the self-reported facilitators could be listed within each of the five major CFIR domains. Trialability (by using real time feedback forms per patient and the quarterly performance audit) and the national collaborative educational sessions (by stimulating peer pressure and networking) were considered as the most important facilitators. The implementation climate of the professionals directly involved in the perioperative process was positively ranked. Nurses particularly appreciated the information given by their local peers and recognized their important role within ERAS. The PDSA cycles and the cyclical multidisciplinary approach contributed to the fact that hospitals maintained their enthusiasm and positively influenced the process of implementation. The process analysis also revealed some barriers to implementation. The perspective that ERAS resulted in extra work load for nurses, mainly during evening hours, resulted in a decreased compliance in mobilization goals. The large number of multidisciplinary professionals (e.g. outpatient clinical nurses, dieticians, physiotherapists and members of the pain management team) involved during the perioperative process complicated implementation. Additional costs related to the implementation of chewing gum and preoperative carbohydrate 
load resulted in another barrier. In some hospitals patients were routinely admitted to the intensive care unit or post anesthesia care unit after surgery. These organizational constraints were seen as barriers to implementation. Although the general department staff adapted existing workflows, almost no adjustment in the workflow on the intensive care unit was reached.

\section{Stepped implementation strategy}

All hospitals received the digital toolbox (phase 1) after the start of the study. However, this did not lead to a direct start of implementation activities at local level. Hospitals assumed that the toolbox would be enough for adequate local implementation. Local stakeholders agreed that their actual care still deviated from ERAS, but decided not to follow the invitation to intensify the support of the expert panel by participating in the optional meetings on national and regional level. The time frame between feedback of real-time performance and the planning of the meetings was too short to attend the meetings in actual practice. In fact, only one hospital was able to attend the meetings of the second and third implementation level. Due to practical reasons, these meetings were combined with the learning sessions of breakthrough implementation group. The fourth and most intensive implementation level was conducted in one hospital. In an outreach visit, members of the expert panel visited local peers to discuss the audit data and to define barriers and facilitators of change. Based on the quarterly audit data, progress was necessary in the intake, mobilization and pain protocol elements of the enhanced recovery elements. The hospital had the ambition to implement ERAS throughout all surgical disciplines; therefore not all protocol elements were adopted yet. This resulted in a positive implementation climate within this hospital. Mainly organizational barriers did hamper the implementation of some of the elements, because elements were not compatible with existing workflows. Admission of patients to the post anesthesia care unit, the responsibility of removal of epidural analgesia by members of the pain management team, and the more preserved policies during the weekend resulted in a delay of the implementation of ERAS principles. During the multidisciplinary meeting solutions to overcome these barriers were provided. We had no insight in local barriers and facilitators of the other hospitals in the stepped implementation group.

\section{DISCUSSION}

In this national cluster randomized controlled trial, including over 1,000 patients, the breakthrough strategy was compared with a new tailored stepped approach 
designed to deliver an optimal effect that is in balance with implementation efforts. The design of this tailored stepped strategy was based on existing evidence and was adaptable to varying clinical settings. The tailored stepped implementation strategy did not differ in effectiveness compared to the breakthrough strategy, resulting in comparable improvement in length of recovery and length of hospital stay between study groups. As could be expected, guideline adherence was the highest at the last implementation phase (phase 4), resulting in an increase in compliance of over $30 \%$ in both study arms compared to outcomes of the baseline audit, before implementation activities had started. Although both strategies improved outcomes on patient level, adjusted analysis showed no differences in effect between the strategies used. Process data showed, however, some contamination to have occurred and poor fidelity in the tailored stepped implementation group. This is mainly due to the complexity of the design of the tailored stepped implementation strategy and our underestimation of this complexity and its time constraints.

Our study did not show the preferred strategy for large-scale implementation of clinical interventions. However, it confirms the importance of evaluating both effect outcomes and process measures of innovative strategies. Although the innovative tailored stepped strategy was well designed and thought through, we encountered a timing problem during execution of the trial. An evaluation moment was planned every three months (at 3, 6, and 9 months) to decide to step up to a higher level of implementation activities, based on an audit of the primary outcome. But this auditing and decision making appeared to take more time than expected. The time constraint was the major limitation for the stepped implementation strategy, limiting fidelity in the tailored stepped implementation arm. The three-month evaluation periods were chosen to follow the timing of the breakthrough educational sessions. It seemed that the time between audit and feedback was too short to plan an additional, next step every three months. The poor fidelity in the tailored stepped implementation group could be considered as the most important limitation in the design of the innovative implementation strategy. Therefore, we decided not to perform the intended cost-minimization analysis as stated in the study protocol [12]. Data collection was carried out by one member of the expert panel. This optimized standardization in the data collection, however could have influenced sustainability because extraction, analysis, and interpretation of own data was no longer the responsibility of the participants and therefore no part of standard care. The study followed an intention to treat analysis to prevent over estimation of the implementation effect and concealed allocation was used to avoid bias.

In this study the guideline for perioperative care in elective pelvic surgery as proposed by the ERAS Society ${ }^{\circledR}$ in 2013 was used as a template [2]. Specific recommendations for gynecologic procedures were first published in 2016 [16, 17], but no 
major differences in elements between both guidelines existed. Recently, an update of this gynecologic guideline became available [18]. Studies have shown that the combination of ERAS elements is more important than implementation of single elements alone $[19,20]$. During the implementation process, it was attempted to implement the whole guideline. However, analysis included a composite score of only twelve index elements. With the selection of these elements, representing pre-, intra-, and postoperative care, it was assumed that a reliable representation of the total ERAS pathway was provided. Recently, a systematic review of 53 studies was conducted to examine the factors that affected ERAS implementation [21]. The key facilitating factors included the possibility to adapt the pathway to local contexts, using a formal implementation framework, and leveraging supporters and fulltime ERAS coordinators [21]. Barriers of success included a resistance to change from health care professionals and a lack of resources for implementation [21]. Although it was not possible to correlate process data to the level of success, findings of our process analyses suggested similar results. In their Delphi study, Francis et al. [22] aimed to draw consensus from an expert panel regarding the successful training and implementation of ERAS. They concluded that successful implementation required strong leadership, an ERAS facilitator, and an effective multidisciplinary team [22]. The concept of our designed stepped implementation strategy included almost all these facilitating factors. The appointment of a fulltime ERAS coordinator is ideal, however due to restricted resources in hospitals almost impossible. These tasks have to be performed by one of the stakeholders involved in the initial implementation project.

In both study groups multifaceted interventions were used to change perioperative practice. By exposing both groups to an implementation strategy, instead of usual care as control, and the focus on effect and process evaluation, the trial had a different approach compared to most of the existing implementation studies [23]. Previous research in other fields showed that the effect of multifaceted strategies for the implementation of best practices in health care is inconsistent. Some studies found a positive effect $[24,25]$, but others do not suggest any additional value of multifaceted strategies to change health care [26, 27]. Tailoring interventions to specific barriers could be promising, however outcomes vary and overall effect sizes are still modest [28]. The shift of a traditional culture towards best practice remains a challenge. Although a momentum for implementation was created in this trial, priority to change differed between health professionals, resulting in a delay in the start of local implementation during the study. This difference between the so-called early adopters, early majority and late majority can be explained by the Rogers's diffusion theory and reflects normal practice. We were unable to detect if this observed delay in implementation efforts significantly influenced research outcomes. 
Based on the results of this randomized controlled trial, we were unable to define the preferred strategy for large-scale implementation of clinical interventions. Both implementation strategies provided comparable results; however some major drawbacks existed. Process analyses of our innovative tailored stepped implementation strategy showed several limitations that hinder actual use in daily practice. The design of a feasible, tailored, and stepped strategy, which could be more appropriate for use in daily practice, should be a topic for further research. 


\section{SUPPLEMENTARY FILES}

Supplementary table (S1) Unadjusted analysis of outcome measures between data of the baseline audit and data obtained during implementation (phase 1-4).

\begin{tabular}{lllllll}
\hline & \multicolumn{2}{l}{ Breakthrough strategy } & \multicolumn{3}{l}{ Stepped strategy } \\
\cline { 2 - 7 } & Baseline & Phase 1-4 & & Baseline & Phase 1-4 \\
\cline { 2 - 7 } & Mean $\pm S D$ & Mean $\pm S D$ & $P$ Value & Mean $\pm S D$ & Mean $\pm S D$ & $P$ Value \\
\hline Protocol compliance (\%) & $42.2 \pm 15.3$ & $67.5 \pm 16.9$ & $<0.001$ & $43.6 \pm 15.6$ & $65.6 \pm 18.3$ & $<0.001$ \\
\hline Length of recovery (days) & $5.1 \pm 2.6$ & $4.3 \pm 2.7$ & $<0.001$ & $4.6 \pm 2.8$ & $4.3 \pm 2.4$ & 0.187 \\
\hline Length of stay (days) & $7.0 \pm 3.5$ & $5.8 \pm 3.9$ & $<0.001$ & $6.3 \pm 3.4$ & $5.8 \pm 3.9$ & 0.142 \\
\hline & & & & & & \\
\hline \% recovered $\leq 3$ days & $50 / 210(23.8)$ & $253 / 642(39.4)$ & $<0.001$ & $74 / 210(35.2)$ & $193 / 456(42.3)$ & 0.083 \\
\hline \% discharged $\leq 5$ days & $86 / 210(41.0)$ & $407 / 642(63.4)$ & $<0.001$ & $112 / 210(53.3)$ & $277 / 456(60.7)$ & 0.071 \\
\hline$S D=$ standard deviation & & & & & &
\end{tabular}

$S D=$ standard deviation.

Supplementary table (S2). Overview of the number of patients included per hospital.

\begin{tabular}{|c|c|c|c|c|c|}
\hline & Phase 1 & Phase 2 & Phase 3 & Phase 4 & Total \\
\hline \multicolumn{6}{|c|}{ Breakthrough implementation } \\
\hline \multicolumn{6}{|c|}{ Level of care: secondary } \\
\hline Hospital 1 & 29 & 27 & 31 & 37 & 124 \\
\hline Hospital 2 & 62 & 54 & 58 & 64 & 238 \\
\hline Hospital 3 & 23 & 19 & 27 & 18 & 87 \\
\hline \multicolumn{6}{|c|}{ Level of care: tertiary } \\
\hline Hospital 4 & 8 & 13 & 16 & 18 & 55 \\
\hline Hospital 5 & 8 & 15 & 10 & 13 & 46 \\
\hline Hospital 6 & 5 & 7 & 7 & 7 & 26 \\
\hline Hospital 7 & 21 & 16 & 19 & 10 & 66 \\
\hline \multicolumn{6}{|c|}{ Stepped implementation } \\
\hline \multicolumn{6}{|c|}{ Level of care: secondary } \\
\hline Hospital 1 & 28 & 26 & 25 & 30 & 109 \\
\hline Hospital 2 & 27 & 27 & 30 & 36 & 120 \\
\hline Hospital 3 & 14 & 5 & 14 & 20 & 53 \\
\hline \multicolumn{6}{|c|}{ Level of care: tertiary } \\
\hline Hospital 4 & 19 & 11 & 10 & 3 & 43 \\
\hline Hospital 5 & 13 & 14 & 7 & 8 & 42 \\
\hline Hospital 6 & 7 & 10 & 10 & 11 & 38 \\
\hline Hospital 7 & 12 & 19 & 12 & 8 & 51 \\
\hline
\end{tabular}




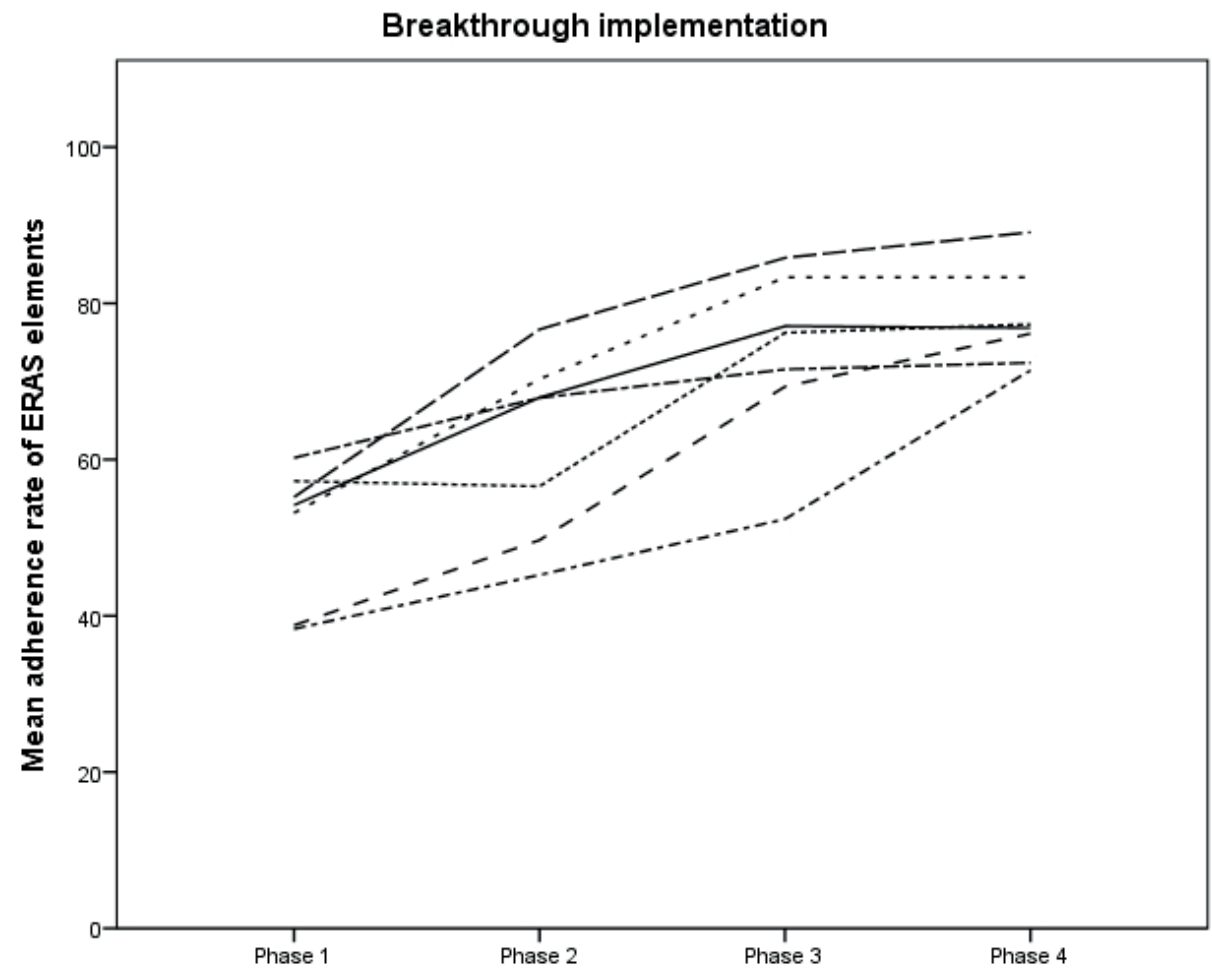

Supplementary figure (S3). Adherence rates to the selected perioperative care index elements per hospital during the implementation phases in the breakthrough implementation group. Each point represents mean values during a 3-month period. The overall mean adherence rate in the breakthrough implementation group at baseline audit (before the start of implementation activities) was $42.2 \pm 15.3 \%$ (data not included) and $67.5 \pm 16.9 \%$ during the four implementation phases. 


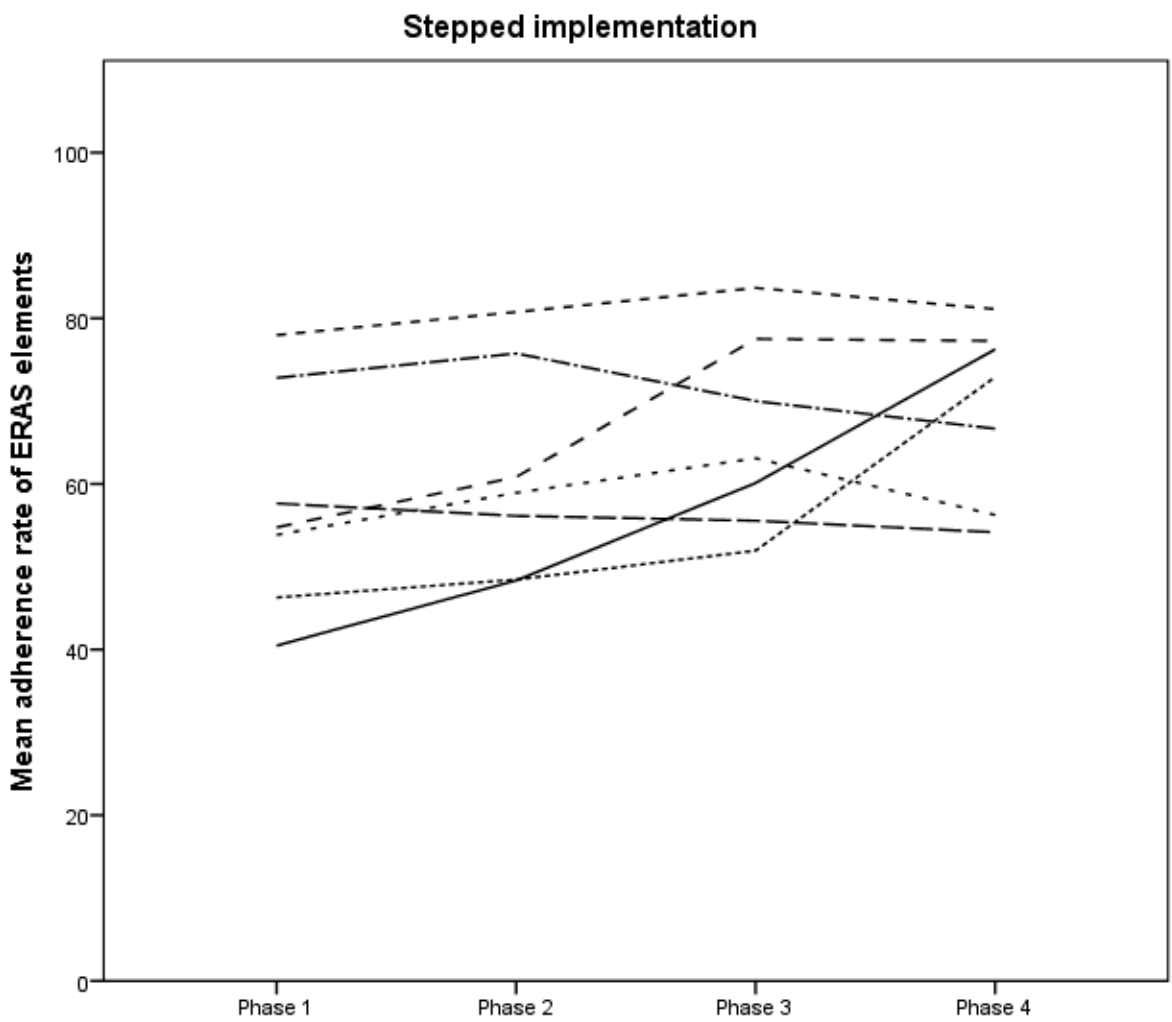

Supplementary figure (S4). Adherence rates to the selected perioperative care index elements per hospital during the implementation phases in the stepped and tailored implementation group. Each point represents mean values during a 3-month period. The overall mean adherence rate in the stepped and tailored implementation group at baseline audit (before the start of implementation activities) was 43.6 $\pm 15.6 \%$ (data not included) and $65.6 \pm 18.3 \%$ during the four implementation phases. 


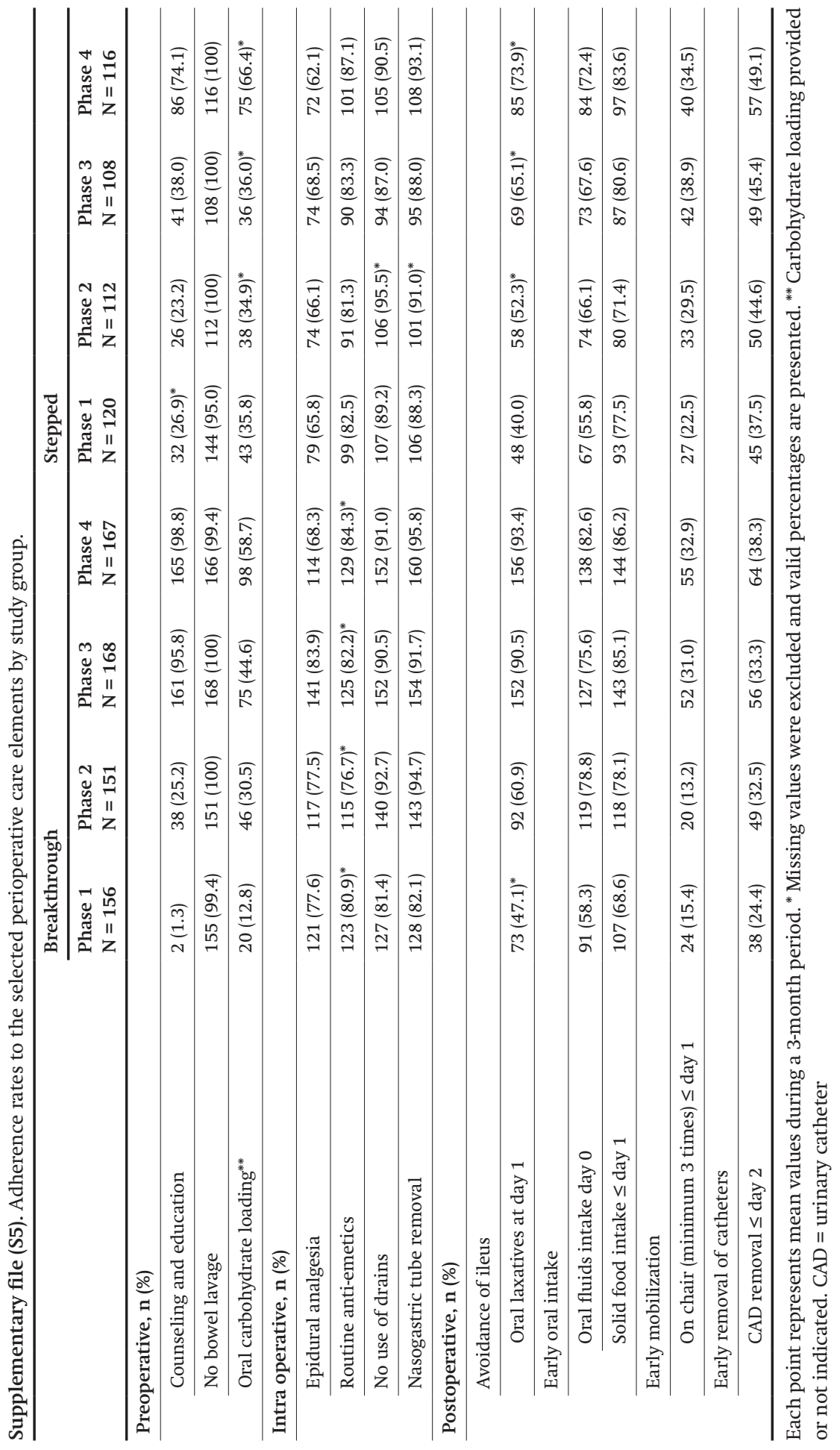




\section{REFERENCES}

1. Ljungqvist O, Scott M, Fearon KC: Enhanced Recovery After Surgery: A Review. JAMA surgery 2017, 152(3):292-298.

2. Nygren J, Thacker J, Carli F, Fearon KC, Norderval S, Lobo DN, Ljungqvist O, Soop M, Ramirez J, Enhanced Recovery After Surgery Society fPC et al: Guidelines for perioperative care in elective rectal/pelvic surgery: Enhanced Recovery After Surgery (ERAS((R))) Society recommendations. World journal of surgery 2013, 37(2):285-305.

3. Visioni A, Shah R, Gabriel E, Attwood K, Kukar M, Nurkin S: Enhanced Recovery After Surgery for Noncolorectal Surgery?: A Systematic Review and Meta-analysis of Major Abdominal Surgery. Annals of surgery 2018, 267(1):57-65.

4. Liu VX, Rosas E, Hwang J, Cain E, Foss-Durant A, Clopp M, Huang M, Lee DC, Mustille A, Kipnis P et al: Enhanced Recovery After Surgery Program Implementation in 2 Surgical Populations in an Integrated Health Care Delivery System. JAMA surgery 2017, 152(7):e171032.

5. Schouten LM, Hulscher ME, van Everdingen JJ, Huijsman R, Grol RP: Evidence for the impact of quality improvement collaboratives: systematic review. Bmj 2008, 336(7659):1491-1494.

6. Nadeem E, Olin SS, Hill LC, Hoagwood KE, Horwitz SM: Understanding the components of quality improvement collaboratives: a systematic literature review. The Milbank quarterly 2013, 91(2):354-394.

7. Improvement IfH: The breakthrough series: IHI's colaborative model for achieving breakthrough improvement. Cambridge, Massachusetts: Institute for Healthcare Improvement.

8. Kilo CM: A framework for collaborative improvement: lessons from the Institute for Healthcare Improvement's Breakthrough Series. Quality management in health care 1998, 6(4):1-13.

9. Curran GM, Bauer M, Mittman B, Pyne JM, Stetler C: Effectiveness-implementation hybrid designs: combining elements of clinical effectiveness and implementation research to enhance public health impact. Medical care 2012, 50(3):217-226.

10. Cochrane Effective Practice and Organisation of Care Review Group: Data Collection Checklist. . In. Edited by Health. IoP. Ontario: University of Ottawa.

11. Newton PJ, Davidson PM, Halcomb EJ, Denniss AR, Westgarth F: An introduction to the collaborative methodology and its potential use for the management of heart failure. The Journal of cardiovascular nursing 2006, 21(3):161-168.

12. de Groot JJ, Maessen JM, Slangen BF, Winkens B, Dirksen CD, van der Weijden T: A stepped strategy that aims at the nationwide implementation of the Enhanced Recovery After Surgery programme in major gynaecological surgery: study protocol of a cluster randomised controlled trial. Implementation science : IS 2015, 10:106.

13. de Groot JJA, Timmermans M, Maessen JMC, Winkens B, Dirksen CD, Slangen BFM, van der Weijden T: Quality improvement strategies for organizational change: a multiphase observational study to increase insight into nonparticipating organizations. BMC health services research 2018, 18(1):1011.

14. Hulscher ME, Laurant MG, Grol RP: Process evaluation on quality improvement interventions. Quality \& safety in health care 2003, 12(1):40-46. 
15. Damschroder LJ, Aron DC, Keith RE, Kirsh SR, Alexander JA, Lowery JC: Fostering implementation of health services research findings into practice: a consolidated framework for advancing implementation science. Implementation science : IS 2009, 4:50.

16. Nelson G, Altman AD, Nick A, Meyer LA, Ramirez PT, Achtari C, Antrobus J, Huang J, Scott M, Wijk L et al: Guidelines for postoperative care in gynecologic/oncology surgery: Enhanced Recovery After Surgery (ERAS(R)) Society recommendations--Part II. Gynecologic oncology 2016, 140(2):323-332.

17. Nelson G, Altman AD, Nick A, Meyer LA, Ramirez PT, Achtari C, Antrobus J, Huang J, Scott M, Wijk L et al: Guidelines for pre- and intra-operative care in gynecologic/oncology surgery: Enhanced Recovery After Surgery (ERAS(R)) Society recommendations--Part I. Gynecologic oncology 2016, 140(2):313-322.

18. Nelson G, Bakkum-Gamez J, Kalogera E, Glaser G, Altman A, Meyer LA, Taylor JS, Iniesta M, Lasala J, Mena G et al: Guidelines for perioperative care in gynecologic/oncology: Enhanced Recovery After Surgery (ERAS) Society recommendations-2019 update. International journal of gynecological cancer : official journal of the International Gynecological Cancer Society 2019.

19. Gustafsson UO, Hausel J, Thorell A, Ljungqvist O, Soop M, Nygren J, Enhanced Recovery After Surgery Study G: Adherence to the enhanced recovery after surgery protocol and outcomes after colorectal cancer surgery. Archives of surgery 2011, 146(5):571-577.

20. Wijk L, Udumyan R, Pache B, Altman AD, Williams LL, Elias KM, McGee J, Wells T, Gramlich L, Holcomb K et al: International validation of Enhanced Recovery After Surgery Society guidelines on enhanced recovery for gynecologic surgery. American journal of obstetrics and gynecology 2019.

21. Stone AB, Yuan CT, Rosen MA, Grant MC, Benishek LE, Hanahan E, Lubomski LH, Ko C, Wick EC: Barriers to and Facilitators of Implementing Enhanced Recovery Pathways Using an Implementation Framework: A Systematic Review. JAMA surgery 2018, 153(3):270-279.

22. Francis NK, Walker T, Carter F, Hubner M, Balfour A, Jakobsen DH, Burch J, Wasylak T, Demartines N, Lobo DN et al: Consensus on Training and Implementation of Enhanced Recovery After Surgery: A Delphi Study. World journal of surgery 2018, 42(7):1919-1928.

23. Davies P, Walker AE, Grimshaw JM: A systematic review of the use of theory in the design of guideline dissemination and implementation strategies and interpretation of the results of rigorous evaluations. Implementation science : IS 2010, 5:14.

24. Grimshaw JM, Eccles MP, Lavis JN, Hill SJ, Squires JE: Knowledge translation of research findings. Implementation science : IS 2012, 7:50.

25. Boaz A, Baeza J, Fraser A, European Implementation Score Collaborative G: Effective implementation of research into practice: an overview of systematic reviews of the health literature. BMC research notes 2011, 4:212.

26. Squires JE, Sullivan K, Eccles MP, Worswick J, Grimshaw JM: Are multifaceted interventions more effective than single-component interventions in changing health-care professionals' behaviours? An overview of systematic reviews. Implementation science : IS 2014, 9:152.

27. Suman A, Dikkers MF, Schaafsma FG, van Tulder MW, Anema JR: Effectiveness of multifaceted implementation strategies for the implementation of back and neck pain guidelines in health care: a systematic review. Implementation science : IS 2016, 11(1):126. 
28. Shaw B, Cheater F, Baker R, Gillies C, Hearnshaw H, Flottorp S, Robertson N: Tailored interventions to overcome identified barriers to change: effects on professional practice and health care outcomes. The Cochrane database of systematic reviews 2005(3):CD005470. 

Quality improvement strategies for

organizational change A multiphase observational study to increase insight into nonparticipating organizations

Jeanny J.A. de Groot

Maite Timmermans

José M.C. Maessen

Bjorn Winkens

Carmen D. Dirksen

Brigitte F.M. Slangen

Trudy van der Weijden

on behalf of the SINERGY nonparticipant group

BMC Health Serv Res 2018; 18:1011 


\begin{abstract}
Background The scope of implementation research is often restricted to the analysis of organizations that participate voluntarily in implementation interventions. The recruitment of participants for a quality improvement collaborative increases awareness of the specific innovation. The objective of this multiphase observational study was to identify differences between organizations that participated in a largescale implementation project aiming to improve perioperative care, functional recovery, and length of hospital stay after gynecologic surgery and organizations that did not participate. A secondary objective was to explore how perioperative practice changed among nonparticipants.
\end{abstract}

Methods Of the seven gynecology departments of nonparticipating Dutch hospitals, five agreed to participate in a retrospective analysis. Baseline data of participating hospitals' $(\mathrm{N}=19)$ characteristics, time to functional recovery, and length of hospital stay were compared. Outcome measures for the subsequent pre-post awareness study in the five nonparticipating hospitals were: (1) overall adherence to predefined evidence-based perioperative elements; and (2) change in functional recovery and length of hospital stay. Multivariable regression models, adjusted for baseline characteristics, were used for analysis.

Results In retrospect, nonparticipating and participating hospitals did not differ in baseline characteristics, functional recovery, and length of hospital. In three of the five nonparticipating hospitals, adherence to the selected evidence-based perioperative elements increased significantly after awareness of the trial (overall mean difference 9.7\%, 95\% CI 6.9\% to 12.5\%, p < 0.001). Linear regression models revealed no statistically significant or clinically relevant differences in time to functional recovery (mean difference -0.2 days, $95 \%$ CI -0.7 to $0.2, \mathrm{p}=0.319$ ) or length of hospital stay (mean difference -0.4 days, $95 \%$ CI -1.3 to $0.5, \mathrm{p}=0.419$ ) in the nonparticipating hospitals. None of these hospitals managed to reduce time to functional recovery or length of hospital stay significantly.

Conclusions No differences in perioperative outcomes between the nonparticipating and participating hospitals were identified at baseline. Despite the statistically significant improvement in overall evidence-based perioperative care, the awareness raised by recruitment activities alone was not enough to reduce time to functional recovery and length of hospital stay in nonparticipating hospitals. Insight into the trends of nonparticipants is valuable to existing implementation effectiveness research. 


\section{BACKGROUND}

Several single and multifaceted implementation interventions have been used to improve the translation of knowledge and guidelines into medical practice [1-3]. These implementation interventions are specified activities designed to enhance adoption of clinical practice with the goal to improve healthcare outcomes $[4,5]$. Their effectiveness in optimizing patient outcomes and healthcare quality has been addressed across different contexts, but further evaluations are still required in order to explore the practical implications of different strategies [2, 3, 6]. Whether beneficial implementation effects on health outcome or quality measures will occur and to what extent is difficult to predict in advance [2, 3, 6]. Moreover, the process of change requires the investment of considerable financial and personal resources $[7,8]$. These considerations have the potential to influence health organizations' choices regarding the execution of implementation interventions [2, 7].

Grol $[9,10]$ described several specific steps and related barriers involved in the process of change. Orientation, insight, and acceptance are necessary before actual change can begin in practice [10]. Studies have shown that organizational readiness facilitates the successful implementation of innovations and new guidelines [11]. In their review article, Weiner and colleagues conclude that both motivation and capability are essential components of readiness for change [11]. There has been increased awareness of the role of such contextual factors on the effectiveness of implementation interventions in healthcare $[12,13]$.

Achieving large-scale implementation of innovations and new guidelines across intra-organizational boundaries is even more complex. Quality improvement collaboratives are designed to improve healthcare quality and outcomes among multiple organizations by using a structured, temporary approach [6, 14]. Sponsorship and the type and priority of the specific issue might determine whether organizations participate in those collaboratives [15]. Participants usually take part in quality improvement collaboratives voluntarily in order to improve quality in healthcare [15]. This depends primarily on their willingness to invest and take part. The scope of implementation research is often restricted to the analysis of participants. Specific recruitment processes remain unexamined and could affect the representativeness of the study sample. Although previous studies have addressed nonparticipation in other settings, the evaluation of sample representativeness is particularly interesting in implementation research. In every process of change, target groups can be divided into several categories according to Rogers's diffusion theory [16]. The process by which an innovation spreads among members of a social system over time is described in this conceptual theory. So, it can be assumed that participants of large-scale implementation strategies differ from nonparticipants. Analysis of 
demographic variables and reasons for nonparticipation could reveal subsequent participation bias.

In Grol's ten-stage model [10], awareness is pointed out as the first orienting step in the process of change. This action model for effective implementation of change formulates a systematic step-wise proposal for change and can be used to plan and support an implementation process. The recruitment of participants in a quality improvement collaborative increases awareness of the specific innovation [17]. Evaluating the influence of this awareness in terms of how it effects change in medical practice among the nonparticipant group might further understandings of implementation processes. In the present study, we focused on the nonparticipants of the 'Stepped Implementation of Enhanced Recovery in Gynecology' (SINERGY) trial. The SINERGY trial is a nationwide comparative effectiveness implementation study that aims to compare two different large-scale implementation strategies across Dutch hospitals in order to define the most efficient strategy to improve practice [18]. A traditional breakthrough strategy with generic, short-term cyclic educational sessions is compared with an innovative stepped implementation strategy comprising different levels of intensity of support adapted to the specific needs of an organization. Reviews have indicated that implementing evidence-based perioperative elements in gynecologic surgery results in improved perioperative outcomes [19, 20, 21].

There were two primary objectives of the current study: (1) to identify differences between the nonparticipating and participating hospitals of the SINERGY trial; (2) to explore whether awareness of the start of a perioperative improvement program in other hospitals affected perioperative outcomes in hospitals that did not participate in the improvement program. The overall group effect on outcome measures and differences in effect within individual hospitals will be evaluated.

\section{METHODS}

\section{Design}

A multiphase observational study was conducted sequentially. To assess the representativeness of the potentially eligible sample of the SINERGY trial, a retrospective observational multicenter approach was used, which compared nonparticipating hospitals with hospitals participating in the SINERGY trial. Subsequently, a pre-post study was conducted in the nonparticipating hospitals in order to identify any changes in perioperative practice, length of functional recovery, and length of postoperative hospital stay. This study was financially supported by the Netherlands Organization for Health Research and Development (ZonMw) (project number: 837003002). 


\section{Setting}

The current study can be seen as an addition to the nationwide SINERGY trial [18]. All the gynecology departments of the 27 hospitals authorized to perform major gynecologic oncologic surgery in the Netherlands were assessed for eligibility. In line with the SINERGY trial protocol [18], one hospital that referred most of its patients to inpatient rehabilitation facilities after hospital discharge was excluded a priori. In total, 26 eligible hospitals were invited to participate in the SINERGY trial.

\section{Recruitment process}

A combination of targeted recruitment efforts were undertaken to increase involvement in the SINERGY trial. In mid-2013 local stakeholders were identified and contacted directly by members of the expert team. A personal letter was sent, which contained information about the study project with the implementation strategies being tested, objectives of the perioperative guidelines, the need for active implementation, and the expected time and personal commitment required. In conformance with the SINERGY trial protocol [18], and in order to minimize implementation effects before the start of the study project, detailed information about the specific elements of the perioperative guidelines were not disseminated. Local stakeholders were, however, aware of the intentions of the SINERGY trial to reduce time to recovery and length of hospital stay by implementing evidence-based perioperative elements according to the enhanced recovery after surgery (ERAS) approach [18]. Reminders by email and telephone were used consecutively, when necessary. Visits to local organizations to provide additional information were undertaken on request. The study was supported by the Dutch Gynecology Oncology Group (DGOG), which comprises professionals who collaborate to promote research in gynecologic cancer. Funding provided by ZonMw helped to tackle financial disincentives by confining the local costs of implementation to attending meetings and executing local activities. Local stakeholders continued to be approached until a statement of commitment or nonparticipation was provided, resulting in a response rate of $100 \%$. Even though all hospitals acknowledged the need for the nationwide standardization of perioperative practice consistent with ERAS, some decided not to participate in the SINERGY trial (nonparticipation rate 27\%). These hospitals did not undergo a baseline audit of perioperative practice to determine if they were eligible for inclusion in the SINERGY trial. Stakeholders were asked about their reasons for nonparticipation. A combination of the compulsory nature of collective multidisciplinary meetings, a lack of time and resources due to high workload, and the lack of financial incentives were reported. In addition, nonparticipants questioned the return on their investment of implementing the evidence-based perioperative elements in improving time to recovery and length of hospital stay. 


\section{Study groups}

In total, seven hospitals chose not to take part in the SINERGY trial. Five of these seven hospitals agreed to participate in the current study. These 'nonparticipating in SINERGY trial' hospitals were compared with eligible participants of the SINERGY trial in order to address the first research objective of determining sample representativeness. The participant group consisted of hospitals that intended to participate in the SINERGY project $(\mathrm{N}=19)$ [18]. For the second research objective, only the nonparticipating hospitals were analyzed for change in perioperative practice and outcomes following recruitment activities for the SINERGY trial. Patients treated in the period before the recruitment process began, and thus before awareness of the SINERGY trial (pre-awareness group), were compared with patients treated one year after awareness of the trial (post-awareness group). This one-year period was chosen because the implementation interventions for the SINERGY trial were to begin in June 2014 [18].

\section{Outcomes}

Nonparticipating and participating hospitals were compared to identify differences in baseline characteristics before the onset of the recruitment process. The total number of beds per hospital, the type of hospital (university or non-university), and the level of specialized health care (secondary or tertiary) were obtained. Effect measures of the implementation of evidence-based perioperative elements were time to functional recovery and length of postoperative hospital stay. The outcomes in these effect measures were assessed in retrospect. Functional recovery was achieved when patients tolerated a normal diet, mobilized independently, and were comfortable on oral analgesia. Day zero refers to the day of surgery.

The outcomes of the second research objective, to explore the effect of awareness, were overall adherence rate to selected evidence-based perioperative care elements, time to functional recovery, and length of postoperative hospital stay in days. The selection of elements was in line with the SINERGY trial protocol [18] and an overall mean adherence rate was calculated. Selected elements included preadmission counseling and education, no bowel lavage, oral carbohydrate loading before surgery, routine use of prophylactic anti-emetics, nasogastric tube removal after surgery, no use of peritoneal drains, oral fluid intake on day zero, start solid food on the first postoperative day, mobilization on chair at least three times on the first postoperative day, use of epidural analgesia, routine use of oral laxatives, and urinary catheter removal before the third postoperative day. These predefined elements can be retrieved reliably and have a direct influence on recovery. In combination they should reflect an enhanced recovery approach. 


\section{Data collection}

In each nonparticipant hospital, retrospective data of two independent, consecutive samples of 30 patients were collected for the pre-awareness (January 2012) and postawareness (June 2014) group. Baseline characteristics, surgical details, and outcome measures were retrieved from medical records. Patients underwent explorative laparotomies, surgical staging, or cytoreductive procedures for suspected, primary, or recurrent gynecologic malignancies. In addition, hospital characteristics were collected. For the participant group the baseline audit included a consecutive sample of 30 patients treated in 2012 . No exclusion criteria were applied at the patient level.

\section{Data analysis}

Absolute numbers with percentages were used for categorical data, while means with standard deviations (SD) or medians with ranges, where appropriate, were used for numerical variables. Differences were assessed using the independent-samples t-test or the Mann-Whitney U test for numerical variables, and the chi-square test or Fisher's exact test, where appropriate, for categorical ones. Both unadjusted and adjusted statistical methods were used to identify differences in implementation endpoints between the nonparticipating and participating hospitals and to assess the overall effect of awareness on perioperative management and outcomes after elective gynecologic surgery. Linear multilevel analysis methods were used to compare the time to functional recovery and the length of hospital stay between the nonparticipating and participating hospitals. Models were adjusted for clustering (random effect) and patient and surgical characteristics (fixed effects). Study group, age, American Society of Anesthesiologists' classification, (suspected) diagnosis and type of surgery, histological subtype, surgical approach, operation time, type of incision, and type of additional surgical procedure were included in the models as fixed effects. To evaluate the overall effect of awareness, the hospitals included in the nonparticipant study could not be treated as a random sample, but were included in the model as a fixed factor. So, multivariable linear regression models, adjusted for patient and surgical characteristics, were used. Furthermore, we were also interested in the separate outcomes for each hospital. Therefore, subsequent analyses of interaction terms between groups (pre-awareness versus post-awareness) and hospitals were performed to determine separate effects. Adjusted mean differences (MD) and 95\% confidence intervals (CI) for results in linear regression models were reported. Multiple imputations (20 imputed datasets) were used to replace missing data on covariates, where appropriate. Since there was only one case with a missing covariate in both the pre-awareness and post-awareness group, complete case analysis was used for the regression models of nonparticipants. In a sensitivity analysis, logarithmic transformation of the data on time to functional recovery and length 
of postoperative hospital stay was used to analyze the influence of right-skewness of data in the nonparticipant analysis. A p-value $\leq 0.05$ was considered statistically significant. A more than one day reduction in time to functional recovery and length of hospital stay (pre-awareness versus post-awareness) were considered clinically relevant. Analyses were performed using IBM SPSS Statistics for Windows, version 21.0 (Armonk, NY, USA).

\section{RESULTS}

\section{Nonparticipating versus participating hospitals}

A total of 24 hospitals were compared, five nonparticipating and 19 participating. Although the nonparticipating hospitals tended to be smaller, no significant differences in the baseline characteristics of the nonparticipating and participating hospitals were found (Table 1). Unadjusted analysis demonstrated no significant differences between hospital groups in the SINERGY trial effect outcomes, that is, in time to functional recovery $(\mathrm{p}=0.205)$ and length of postoperative hospital stay $(\mathrm{p}=$ 0.659) at baseline (Table 1). Linear multilevel analysis of time to functional recovery demonstrated an adjusted mean difference of 0.3 (95\% CI - 0.2 to 0.8 ) days ( $\mathrm{p}=0.256$ ) between the nonparticipating and the participating hospitals. The adjusted mean difference in length of postoperative hospital stay was 0.0 (95\% CI -0.8 to 0.8 ) days $(\mathrm{p}=0.977)$.

Table 1. Characteristics of participating and nonparticipating hospitals to the SINERGY trial ( $\mathrm{N}=24)$.

\begin{tabular}{llll}
\hline & $\begin{array}{l}\text { Participating } \\
\text { hospitals } \\
(\mathbf{N}=19)\end{array}$ & $\begin{array}{l}\text { Nonparticipating } \\
\text { hospitals } \\
(\mathbf{N}=5)\end{array}$ & $p$ value \\
\hline Total number of hospital beds, median [range] & $715[367-1320]$ & $653[180-976]$ & $0.455^{\mathrm{a}}$ \\
\hline Type of hospital, N (\%) & & & $>0.999^{\mathrm{b}}$ \\
\hline University medical center & $6(31.6)$ & $1(20.0)$ & $0.631^{\mathrm{b}}$ \\
\hline Non university teaching hospital & $13(68.4)$ & $4(80.0)$ & \\
\hline Level of care, N (\%) & & & $4(80.0)$ \\
\hline Secondary care & $12(63.2)$ & $1(20.0)$ & $0.205^{\mathrm{a}}$ \\
\hline Tertiary care & $7(36.8)$ & & \\
\hline Time to functional recovery (days) & & $5.0[3.0-6.0]$ & \\
\hline Unadjusted, median [range] & $4.0[3.0-5.0]$ & & $0.659^{\mathrm{a}}$ \\
\hline Length of postoperative hospital stay (days) & & $5.0[3.0-7.0]$ & \\
\hline Unadjusted, median [range] & $6.0[4.0-10.5]$ & & \\
\hline
\end{tabular}

Group differences were tested using the Mann-Whitney U test ${ }^{\mathrm{a}}$ or Fisher's exact test ${ }^{\mathrm{b}}$ at hospital level. $\mathrm{N}=$ number of hospitals. 


\section{Pre-awareness versus post-awareness group}

In the nonparticipating hospitals, the medical records of a total of 300 patients who underwent elective gynecologic surgery were collected for the pre-awareness $(n=150)$ and post-awareness $(n=150)$ groups. Patient characteristics and surgical details for the patients treated at baseline (pre-awareness) and post-awareness are shown in Table 2.

Characteristics were similar in terms of American Society of Anesthesiologists' classification, (suspected) diagnosis, and histological subtype; however, mean ( \pm SD) patient age was significantly higher in the post-awareness group (61.8 \pm 12.2 versus $65.1 \pm 12.7$ years, $p=0.024$ ). Surgical approach, length of surgery, and the presence of additional procedures did not differ statistically between the study groups. More patients in the post-awareness group underwent primary cytoreductive surgery for ovarian cancer rather than an explorative or staging procedure compared to the pre-awareness group $(\mathrm{p}=0.027)$.

Adherence rates to the perioperative elements of the ERAS pathway pre- and postawareness are shown in Table 3. Anti-emetics were routinely used during surgery in $61.6 \%(n=90)$ of patients in the pre-awareness group compared with $74.7 \%(n=112)$ of patients in the post-awareness group $(\mathrm{p}=0.016)$. In addition, a significant increase was observed in adherence rates for the avoidance of intraperitoneal drains $(69.3 \%$ versus $81.9 \%, \mathrm{p}=0.011$ ), early oral intake of fluids $(24.7 \%$ versus $42.6 \%, \mathrm{p}=0.001)$ and solid food (55.3\% versus $77.7 \%, \mathrm{p}<0.001)$, and early mobilization on postoperative day one (14.1 versus $27.3 \%, \mathrm{p}=0.005)$. On the other hand, slightly more patients were pretreated with oral bowel lavage solutions $(0.0 \%$ versus $5.4 \%, \mathrm{p}=0.003)$. Overall, there was a significant difference in unadjusted adherence rates to the 12 predefined perioperative elements between the pre- and post-awareness group (mean rate $46.1 \pm$ 15.5 versus $53.3 \pm 13.3, \mathrm{p}<0.001$ ) (Table 4). Multivariable linear regression, adjusted for baseline characteristics and surgical approach, demonstrated a similar result (MD $9.7 \%, 95 \%$ CI $6.9 \%$ to $12.5 \%, \mathrm{p}<0.001)$. A significant increase in the adjusted mean difference in adherence rate was observed for three out of five hospitals (Hospital 2: $\mathrm{p}<$ 0.001, Hospital 3: $\mathrm{p}=0.001$, and Hospital 5: $\mathrm{p}<0.001$ ) (Fig. 1). There was no significant difference in adherence rates to the predefined perioperative elements between the two study groups for Hospital 1 ( $\mathrm{p}=0.921$ ) and Hospital 4 ( $\mathrm{p}=0.875)$ (Fig. 1).

Functional recovery data were obtained for 294 patients $(98.0 \%)$. In the pre-awareness group three patients died during admission (2.0\%). In the post-awareness group one patient died shortly after discharge $(0.7 \%)(\mathrm{p}=0.622)$. The mean $( \pm \mathrm{SD})$ time to functional recovery was $4.8 \pm 2.4$ days in the pre-awareness and $4.9 \pm 2.2$ days in the post-awareness group $(\mathrm{p}=0.892)$ (Table 4$)$. Adjusted analysis of time to functional recovery demonstrated a non-significant reduction of 0.2 days ( $95 \% \mathrm{CI}-0.7$ to $0.2, \mathrm{p}=$ 0.319 ) (Table 4). The effect of awareness on time to functional recovery did not differ significantly for any hospital (Fig. 2). 
No statistically significant or clinically relevant difference in the unadjusted mean length of hospital stay after surgery was observed between the pre- and postawareness group (6.4 \pm 4.2 versus $6.5 \pm 4.1$ days, $\mathrm{p}=0.874$ ) (Table 3). Multivariable linear regression showed that none of the hospitals had a statistically significant change in the length of postoperative hospital stay (Fig. 3). The overall adjusted mean difference remained non-significant (MD -0.4 days, 95\% CI -1.3 to 0.5, $\mathrm{p}=0.419$ ) (Table 4).

Table 2. Baseline characteristics and surgical details of the pre-awareness and post-awareness group.

\begin{tabular}{|c|c|c|c|}
\hline & $\begin{array}{l}\text { Pre-awareness } \\
(\mathrm{n}=150)\end{array}$ & $\begin{array}{l}\text { Post-awareness } \\
(\mathrm{n}=150)\end{array}$ & $p$ value \\
\hline Age in years, mean $\pm S D$ & $61.8 \pm 12.2$ & $65.1 \pm 12.7$ & $0.024^{\mathrm{a}}$ \\
\hline ASA classification, $\mathbf{n}(\%)$ & & & $0.757^{\mathrm{b}}$ \\
\hline $\mathrm{I} / \mathrm{II}$ & $124(82.7)$ & $126(84.0)$ & \\
\hline III/IV & $26(17.3)$ & $24(16.0)$ & \\
\hline (Suspected) diagnosis, n (\%) & & & $0.110^{\mathrm{b}}$ \\
\hline Ovarian cancer & $115(76.7)$ & $114(76.0)$ & \\
\hline Uterine cancer & $27(18.0)$ & $34(22.7)$ & \\
\hline Cervical cancer & $8(5.3)$ & $2(1.3)$ & \\
\hline Histological subtype, n (\%) & & & $0.111^{\mathrm{b}}$ \\
\hline Benign & $37(24.7)$ & $27(18.0)$ & \\
\hline Borderline / hyperplasia & $14(9.3)$ & $8(5.3)$ & \\
\hline Malignant & $99(66.0)$ & $115(76.7)$ & \\
\hline Surgical approach, n (\%) & & & $>0.999^{c}$ \\
\hline Midline incision & $147(98.0)$ & $147(98.0)$ & \\
\hline Transverse incision & $3(2.0)$ & $3(2.0)$ & \\
\hline Operation time in minutes, mean $\pm \mathrm{SD}$ & $138.9 \pm 72.0$ & $142.7 \pm 67.5$ & $0.632^{\mathrm{a}}$ \\
\hline Type of surgery (only ovarian included), n (\%) & & & $0.027^{\mathrm{b}}$ \\
\hline Explorative & $50(43.5)$ & $40(35.1)$ & \\
\hline Staging & $13(11.3)$ & $6(5.3)$ & \\
\hline Primary cytoreductive & $11(9.6)$ & $25(21.9)$ & \\
\hline Secondary cytoreductive & $41(35.7)$ & $43(37.7)$ & \\
\hline \multicolumn{4}{|l|}{ Additional surgical procedure, $\mathbf{n}(\%)$} \\
\hline Open-close (inoperable) & $1(0.7)$ & $5(3.3)$ & $0.214^{\mathrm{c}}$ \\
\hline Anastomosis & $6(4.0)$ & $7(4.7)$ & $0.777^{\mathrm{b}}$ \\
\hline Diverting stoma & $6(4.0)$ & $9(6.0)$ & $0.427^{\mathrm{b}}$ \\
\hline Lymphadenectomy & $34(22.7)$ & $27(18.0)$ & $0.315^{\mathrm{b}}$ \\
\hline Extensive upper abdominal surgery * & $4(2.7)$ & $8(5.3)$ & $0.239^{\mathrm{b}}$ \\
\hline
\end{tabular}

Group differences were tested using the independent samples t-test ${ }^{\mathrm{a}}$, Chi-square test ${ }^{\mathrm{b}}$, or Fisher's exact test $^{c}$. ASA $=$ American Society of Anesthesiologists, $\mathrm{n}=$ number of patients, $\mathrm{SD}=$ standard deviation. ${ }^{*}$ Includes diaphragmatic stripping, partial liver resection, or splenectomy. 
Table 3. Adherence rates to the selected perioperative care elements.

\begin{tabular}{|c|c|c|c|}
\hline & $\begin{array}{l}\text { Pre-awareness } \\
(\mathrm{n}=150)\end{array}$ & $\begin{array}{l}\text { Post-awareness } \\
(\mathrm{n}=150)\end{array}$ & $p$ value $^{\mathrm{a}}$ \\
\hline \multicolumn{4}{|l|}{ Preoperative items, n (\%) } \\
\hline Counseling and education & $0(0)$ & $0(0)$ & \\
\hline No bowel lavage & $150(100)$ & $140(94.6)$ & $0.003^{\mathrm{b}}$ \\
\hline Oral carbohydrate loading (if indicated) & $32(21.3)$ & $35(23.3)$ & 0.782 \\
\hline \multicolumn{4}{|l|}{ Intraoperative items, $\mathbf{n}(\%)$} \\
\hline Epidural analgesia & $121(80.7)$ & $126(84.0)$ & 0.451 \\
\hline Routine anti-emetics & $90(61.6)$ & $112(74.7)$ & 0.016 \\
\hline No use of drains & $104(69.3)$ & $122(81.9)$ & 0.011 \\
\hline Nasogastric tube removal & $105(70.0)$ & $112(75.7)$ & 0.272 \\
\hline \multicolumn{4}{|l|}{ Postoperative items, n (\%) } \\
\hline \multicolumn{4}{|l|}{ Avoidance of ileus } \\
\hline Oral laxatives at day 1 & $33(22.0)$ & $42(28.0)$ & 0.232 \\
\hline \multicolumn{4}{|l|}{ Early oral intake } \\
\hline Oral fluids intake day 0 & $37(24.7)$ & $63(42.6)$ & 0.001 \\
\hline Solid food intake $\leq$ day 1 & $83(55.3)$ & $115(77.7)$ & $<0.001$ \\
\hline \multicolumn{4}{|l|}{ Early mobilization } \\
\hline On chair (minimum 3 times) $\leq$ day 1 & $21(14.1)$ & $41(27.3)$ & 0.005 \\
\hline \multicolumn{4}{|l|}{ Early removal of catheters } \\
\hline $\mathrm{CAD}$ removal $\leq$ day 2 & $51(34.2)$ & $47(31.5)$ & 0.623 \\
\hline
\end{tabular}

Values are valid percentages (excluding missing values). ${ }^{a}$ Chi-square test. ${ }^{\mathrm{b}}$ Fisher's exact test. CAD = urinary catheter, $\mathrm{n}=$ number of patients.

Table 4. Unadjusted and adjusted outcomes for the pre-awareness and post-awareness group.

\begin{tabular}{|c|c|c|c|c|}
\hline & $\begin{array}{l}\text { Pre-awareness } \\
(\mathrm{n}=150)\end{array}$ & $\begin{array}{l}\text { Post-awareness } \\
(\mathrm{n}=150)\end{array}$ & $\begin{array}{l}\text { Mean difference } \\
(95 \% \mathrm{CI})\end{array}$ & $p$ value \\
\hline \multicolumn{5}{|l|}{ Adherence to key items (\%) } \\
\hline Unadjusted mean \pm SD & $46.1 \pm 15.5$ & $53.3 \pm 13.3$ & $7.3(4.0$ to 10.5$)$ & $<0.001^{\mathrm{a}}$ \\
\hline Adjusted EMM (95\% CI) & $54.3(41.5$ to 67.1$)$ & 64.0 (51.2 to 76.9$)$ & 9.7 (6.9 to 12.5$)$ & $<0.001^{\mathrm{b}}$ \\
\hline \multicolumn{5}{|l|}{ Time to recovery (days) } \\
\hline Unadjusted mean \pm SD & $4.8 \pm 2.4$ & $4.9 \pm 2.2$ & $0.0(-0.5$ to 0.6$)$ & $0.892^{\mathrm{a}}$ \\
\hline Adjusted EMM (95\% CI) & $4.9(2.7$ to 7.0$)$ & $4.6(2.5$ to 6.8$)$ & $-0.2(-0.7$ to 0.2$)$ & $0.319^{\mathrm{b}}$ \\
\hline \multicolumn{5}{|c|}{ Length of hospital stay (days) } \\
\hline Unadjusted mean \pm SD & $6.4 \pm 4.2$ & $6.5 \pm 4.1$ & $0.1(-0.9$ to 1.0$)$ & $0.874^{\mathrm{a}}$ \\
\hline Adjusted EMM (95\% CI) & $6.6(2.6$ to 10.7$)$ & $6.3(2.2$ to 10.3$)$ & $-0.4(-1.3$ to 0.5$)$ & $0.419^{\mathrm{b}}$ \\
\hline
\end{tabular}

Group differences were tested using the independent samples t-test ${ }^{a}$, or multivariable linear regression models ${ }^{b}$. Linear models were adjusted for age, ASA classification, diagnosis and type of surgery, histological subtype, surgical approach, duration of surgery, and type of additional surgical procedure. Analysis of the log-transformed data on time to recovery and length of hospital stay showed similar results. $\mathrm{CI}=$ confidence interval, $\mathrm{EMM}=$ estimated marginal mean, $\mathrm{n}=$ number of patients, $\mathrm{SD}=$ standard deviation. 


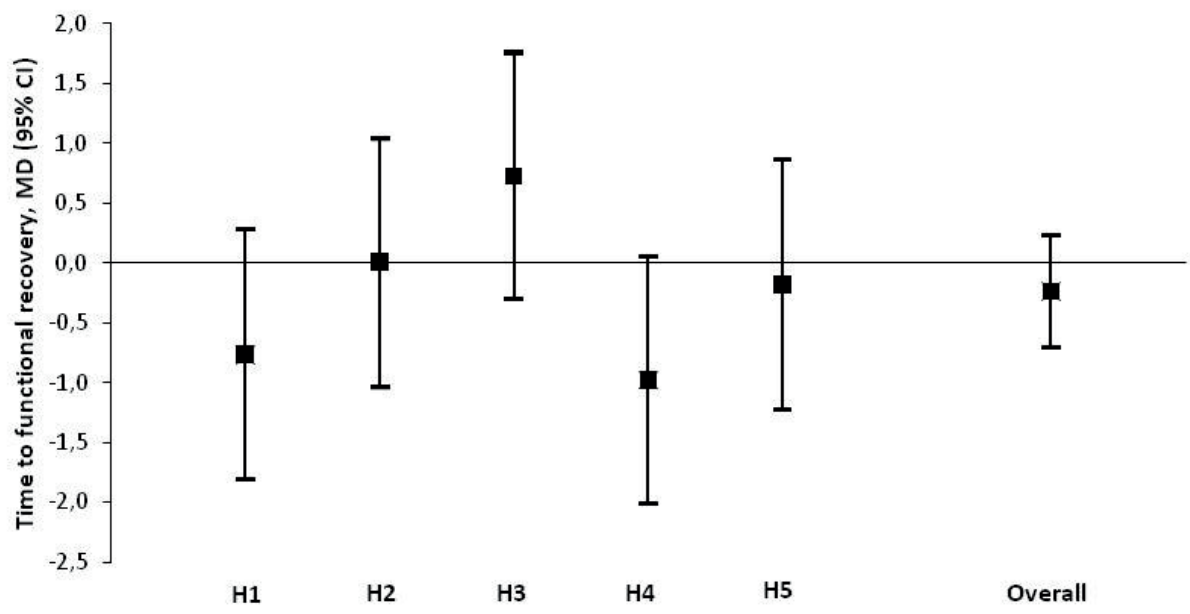

Figure 1. Mean differences in adherence rate to the preselected perioperative elements per hospital with $95 \%$ confidence intervals between the pre-awareness and post-awareness group. Multivariable linear regression models were adjusted for age, ASA classification, diagnosis and type of surgery, histological subtype, surgical approach, duration of surgery, and type of additional surgical procedure. Hospital identifiers were entered in the models as dummy variables with interaction terms.

* indicates statistically significant difference. $\mathrm{CI}=$ confidence interval, $\mathrm{MD}=$ mean difference.

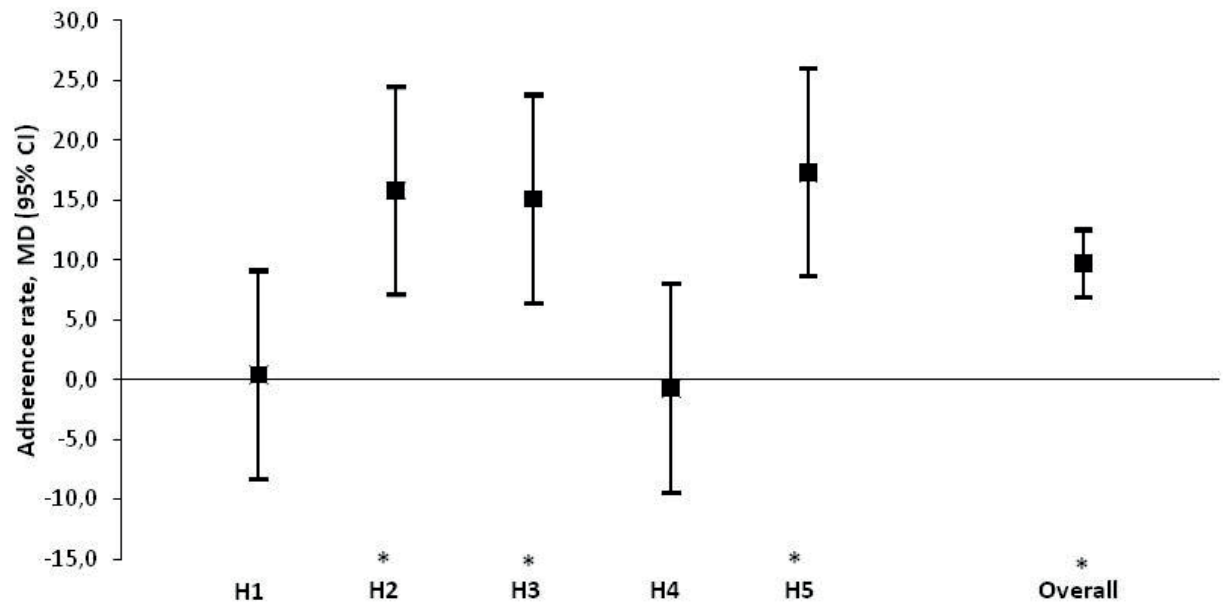

Figure 2. Mean differences in time to functional recovery (days) per hospital with 95\% confidence intervals between the pre-awareness and post-awareness group. Multivariable linear regression models were adjusted for age, ASA classification, diagnosis and type of surgery, histological subtype, surgical approach, duration of surgery, and type of additional surgical procedure. Hospital identifiers were entered in the models as dummy variables with interaction terms. Analysis of the log-transformed data showed similar results. No statistically significant differences were found . $\mathrm{CI}=$ confidence interval, $\mathrm{MD}$ $=$ mean difference. 


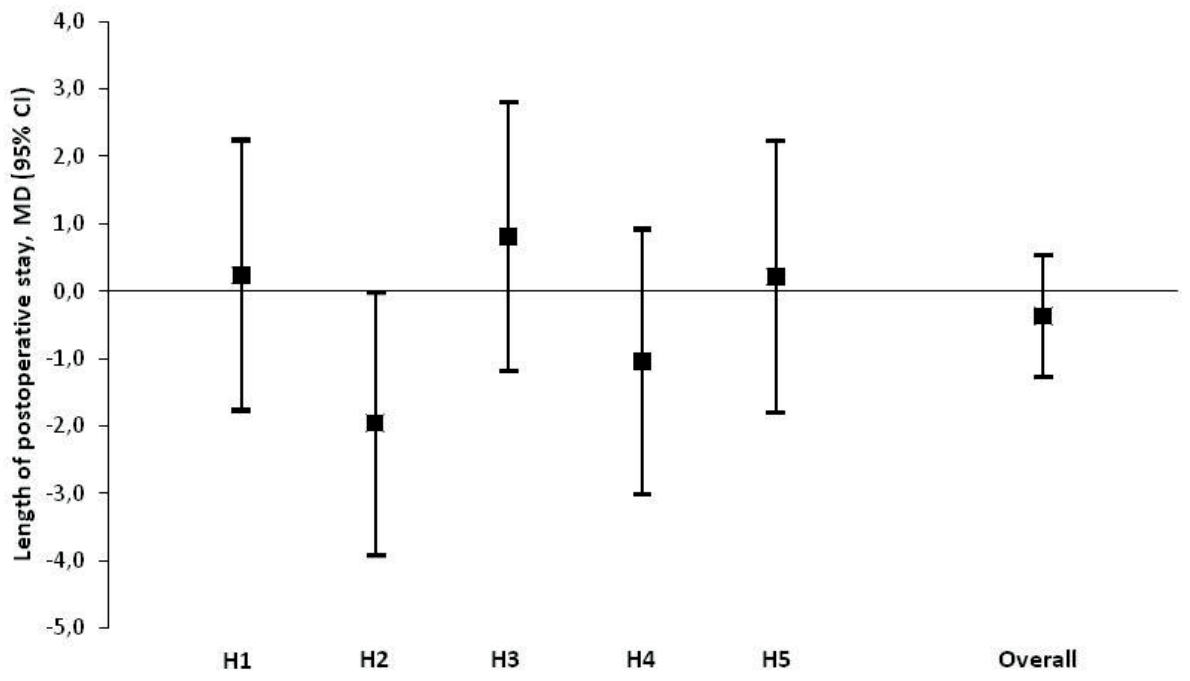

Figure 3. Mean differences in length of postoperative stay (days) per hospital with $95 \%$ confidence intervals between the pre-awareness and post-awareness group. Multivariable linear regression models were adjusted for age, ASA classification, diagnosis and type of surgery, histological subtype, surgical approach, duration of surgery and type of additional surgical procedure. Hospital identifiers were entered in the models as dummy variables with interaction terms. Analysis of the log-transformed data showed similar results. No statistically significant differences were found CI $=$ confidence interval, $\mathrm{MD}$ = mean difference.

\section{DISCUSSION}

In this study, we had the opportunity to illustrate the characteristics of and changes in organizations that did not participate in a nationwide comparative effectiveness implementation trial to implement evidence-based perioperative guidelines across gynecology departments in the Netherlands [18]. The findings provide insight into the effect that awareness of the start of an implementation program can have on the perioperative outcomes of hospitals not participating in the improvement program.

The current study found a significant change in overall perioperative practice, resulting in an estimated mean adherence of $64.0 \%$ (95\% CI $51.2 \%$ to $76.9 \%$ ) to the selected evidence-based elements in the post-awareness group. Analysis of the individual perioperative elements showed that more routine anti-emetics were administered and less intraperitoneal drains were used during surgery. In addition, an increase in early oral intake and early mobilization rates was found. Nevertheless, no statistically significant or clinically meaningful reduction in time to functional recovery or length of postoperative hospital stay occurred in the post-awareness group (MD -0.2 days, $\mathrm{p}=0.319$ and MD -0.4 days, $\mathrm{p}=0.419$, respectively). These results support previous research findings regarding guidelines for enhanced recovery 
after surgery, which indicate a 70\% cutoff as the minimum level of adequate adherence $[22,23]$. The enhanced recovery after surgery guidelines developed by the ERAS Society ${ }^{\circledR}$ include over 20 elements that, according to the best evidence available, are necessary for optimal perioperative care $[24,25]$. The guidelines are used to reduce the gap between research and practice and aim to minimize the stress response to surgery [26]. As a result, functional recovery and time to discharge are supposed to improve. In accordance with the SINERGY trial protocol [18], we analyzed adherence to 12 of these perioperative elements. These predefined elements can be retrieved reliably and have a direct influence on recovery. In combination they should reflect an enhanced recovery approach. However, perioperative data were obtained retrospectively through the review of medical records, which is a limitation of the study.

On a hospital level, three out of five hospitals achieved a significant improvement in evidence-based perioperative care. However, none of them reached the $70 \%$ cutoff and none managed to reduce the effect outcomes on time to functional recovery and length of hospital stay significantly. So, while there is some evidence that local stakeholder awareness of this intended change in perioperative practice might have affected the implementation of single evidence-based elements in nonparticipating hospitals, the improvement did not significantly or clinically change time to functional recovery or length of postoperative hospital stay. These findings are in agreement with Grol's theory regarding the several steps involved in the process of change [10]. Merely increasing awareness through external peers during recruitment activities does not seems sufficient in starting the cascade of change needed to implement complex, multimodal innovations.

Based on Rogers's diffusion theory [16], participating organizations probably belong to the early adopter and early majority categories. These organizations already have the best starting position for achieving beneficial implementation effects compared to nonparticipating organizations. The lack of clinical effect might be explained by differences in resources and willingness to change. Research on the effect of strategies used to implement innovations or guidelines should, therefore, not present data about the volunteering study sample only [27]. Additional information about nonparticipating organizations and contextual factors is particularly necessary to provide representative effect outcomes of different implementation strategies [27]. Mays et al. [28] showed that the public health organizations involved in research networks tend to be more engaged in implementation activities. Based on their findings, the authors suggest that the success of research networks is not only due to selection bias during the recruitment phase, but also to the development of competences during participation in research networks [28]. The findings of this quantitative study do not give insight into the underlying processes involved. There- 
fore, the limited effect could be due to the combination of a preexisting resistance to change and to a lack of resources and tools for achieving organizational change.

Organizational readiness seems to be essential in the process of change [11]. On the other hand, components of organizational readiness determine whether organizations become participants in implementation interventions [15]. In the beginning, we intended to include all eligible hospitals in the SINERGY trial. The process of recruiting hospitals was slow and difficult, despite the use of targeted strategies and facilitators. An evidence-based innovation that had already been proved effective, local stakeholders, peer pressure, and general funding of implementation strategies were used to increase participation. Eventually, a participation rate of $73 \%$ of all eligible hospitals was achieved. Our results suggest that the decision not to participate was based on organizational and financial constraints. This is in line with factors identified by a recent qualitative report [29]. In retrospect, because baseline outcomes in effect measures did not differ between participating and nonparticipating organizations they appear not to have influenced the choice to participate in implementation interventions. Prior studies have determined the importance of addressing local barriers to change during implementation interventions [9]. To increase organizational readiness and the extent of implementation effects, barriers for participating in large-scale implementation interventions also have to be taken into account. In addition, targeted implementation interventions based on specific organizational needs might be effective in increasing participation rates. Organizational readiness is difficult to quantify, therefore qualitative data could have provided more information and could have helped to interpret quantitative findings.

Our findings give insight into the characteristics of and changes that took place in organizations that did not participate in a nationwide comparative effectiveness trial, but they must be interpreted with some caution. Despite the nationwide focus, only a relatively small number of hospitals could be included because of the specific study design. Multivariable regression analyses, with the hospital as a fixed instead of random factor, were used to explore the influence of awareness on perioperative care and outcomes. Therefore, the results are specific to our study sample and regression outcomes lack statistical generalizability. Regardless, we think that the perceived challenges of the recruitment phase and the observed trends in our study might be illustrative of other situations as well, particularly due to its nationwide focus. Data were retrieved from a consecutive sample of patients per hospital. To increase comparability between hospitals all patients included in the study underwent surgery within the same one-year period. Inclusion of a larger number of patients per hospital would have resulted in a longer study period, thereby increasing the chance of bias. 


\section{CONCLUSIONS}

This multiphase observational study has shown that neither hospital characteristics nor perioperative outcomes at baseline differed, in retrospect, between the participating and nonparticipating departments of a national large-scale implementation project. Although a statistically significant improvement in evidence-based perioperative practice was achieved over time, the awareness raised by recruitment activities alone was not enough to facilitate change in functional recovery and length of hospital stay in the nonparticipating hospitals. Despite its exploratory nature, this study offers some insight into the influence of large-scale implementation projects on clinical practice in nonparticipating hospitals. 


\section{REFERENCES}

1. Grimshaw JM, Eccles MP, Lavis JN, Hill SJ, Squires JE. Knowledge translation of research findings. Implement Sci. 2012;7:50.

2. Grimshaw JM, Thomas RE, MacLennan G, Fraser C, Ramsay CR, Vale L, et al. Effectiveness and efficiency of guideline dissemination and implementation strategies. Health Technol Assess. 2004;8(6):iii-v 1-72.

3. Gagliardi AR, Alhabib S, members of Guidelines International Network Implementation Working Group. Trends in guideline implementation: a scoping systematic review. Implement Sci. 2015;10:54.

4. Stetler CB, Mittman BS, Francis J. Overview of the VA Quality Enhancement Research Initiative (QUERI) and QUERI theme articles: QUERI series. Implement Sci. 2008;3:8.

5. Cochrane Effective Practice and Organisation of Care Review Group. Data collection checklist. Ontario: Institute of Population Health. University of Ottawa; 2011.

6. Schouten LM, Hulscher ME, van Everdingen JJ, Huijsman R, Grol RP. Evidence for the impact of quality improvement collaboratives: systematic review. BMJ. 2008;336(7659):1491-4.

7. Gagliardi AR. "More bang for the buck": exploring optimal approaches for guideline implementation through interviews with international developers. BMC Health Serv Res. 2012;12:404.

8. Solberg LI. If you've seen one quality improvement collaborative. Ann Fam Med. 2005;3(3):198-9.

9. Grol R, Wensing M. What drives change? Barriers to and incentives for achieving evidence-based practice. Med J Aust. 2004;180(Suppl 6):S57-60.

10. Grol R. Implementing guidelines in general practice care. Qual Health Care. 1992;1(3):184-91.

11. Weiner BJ, Amick H, Lee SY. Conceptualization and measurement of organizational readiness for change: a review of the literature in health services research and other fields. Med Care Res Rev. 2008;65(4):379-436.

12. Kringos DS, Sunol R, Wagner C, Mannion R, Michel P, Klazinga NS, et al. The influence of context on the effectiveness of hospital quality improvement strategies: a review of systematic reviews. BMC Health Serv Res. 2015;15:277.

13. Ovretveit J. Understanding the conditions for improvement: research to discover which context influences affect improvement success. BMJ Qual Saf. 2011;20(Suppl 1):i18-23.

14. Kilo CM. A framework for collaborative improvement: lessons from the Institute for Healthcare Improvement's breakthrough series. Qual Manag Health Care. 1998;6(4):113.

15. Wilson T, Berwick DM, Cleary PD. What do collaborative improvement projects do? Experience from seven countries. Jt Comm J Qual Saf. 2003; 29(2):85-93.

16. Rogers E. Diffusion of innovations. 5th ed. New York: Free Press; 2003.

17. Maessen J, Dejong CH, Hausel J, Nygren J, Lassen K, Andersen J, et al. A protocol is not enough to implement an enhanced recovery programme for colorectal resection. $\mathrm{Br} \mathrm{J}$ Surg. 2017;94(2):224-31.

18. de Groot JJ, Maessen JM, Slangen BF, Winkens B, Dirksen CD, van der Weijden T. A stepped strategy that aims at the nationwide implementation of the enhanced recovery 
after surgery programme in major gynaecological surgery: study protocol of a cluster randomized controlled trial. Implement Sci. 2015;10:106.

19. Barber EL, Van Le L. Enhanced recovery pathways in gynecology and gynecologic oncology. Obstet Gynecol Surv. 2015;70(12):780 -92.

20. de Groot JJ, Ament SM, Maessen JM, Dejong CH, Kleijnen JM, Slangen BF. Enhanced recovery pathways in abdominal gynecologic surgery: a systematic review and metaanalysis. Acta Obstet Gynecol Scand. 2016;95(4):382 -95.

21. Nelson G, Kalogera E, Dowdy SC. Enhanced recovery pathways in gynecologic oncology. Gynecol Oncol. 2014;135(3):586 -94.

22. Ahmed J, Khan S, Gatt M, Kallam R, MacFie J. Compliance with enhanced recovery programmes in elective colorectal surgery. Br J Surg. 2010;97(5):754 -8.

23. Gustafsson UO, Hausel J, Thorell A, Ljungqvist O, Soop M, Nygren J, et al. Adherence to the enhanced recovery after surgery protocol and outcomes after colorectal cancer surgery. Arch Surg. 2011;146(5):571 -7.

24. Nelson G, Altman AD, Nick A, Meyer LA, Ramirez PT, Achtari C, et al. Guidelines for pre- and intra-operative care in gynecologic/oncology surgery: enhanced recovery after surgery (ERAS®) society recommendations - part I. Gynecol Oncol. 2016;140(2):313 -22.

25. Nelson G, Altman AD, Nick A, Meyer LA, Ramirez PT, Achtari C, et al. Guidelines for postoperative care in gynecologic/oncology surgery: enhanced recovery after surgery (ERAS®) society recommendations - part II. Gynecol Oncol. 2016;140(2):323 -32.

26. Kehlet H. Multimodal approach to control postoperative pathophysiology and rehabilitation. Br J Anaesth. 1997;78(5):606 -17.

27. Glasgow RE, Klesges LM, Dzewaltowski DA, Estabrooks PA, Vogt TM. Evaluating the impact of health promotion programs: using the RE-AIM framework to form summary measures for decision making involving complex issues. Health Educ Res. 2006;21(5):688 -94 .

28. Mays GP, Hogg RA, Castellanos-Cruz DM, Hoover AG, Fowler LC. Public health research implementation and translation: evidence from practicebased research networks. Am J Prev Med. 2013;45(6):752-62.

29. Herbert G, Sutton E, Burden S, Lewis S, Thomas S, Ness A, et al. Healthcare professionals ' views of the enhanced recovery after surgery programme: a qualitative investigation. BMC Health Serv Res. 2017;17(1):617. 




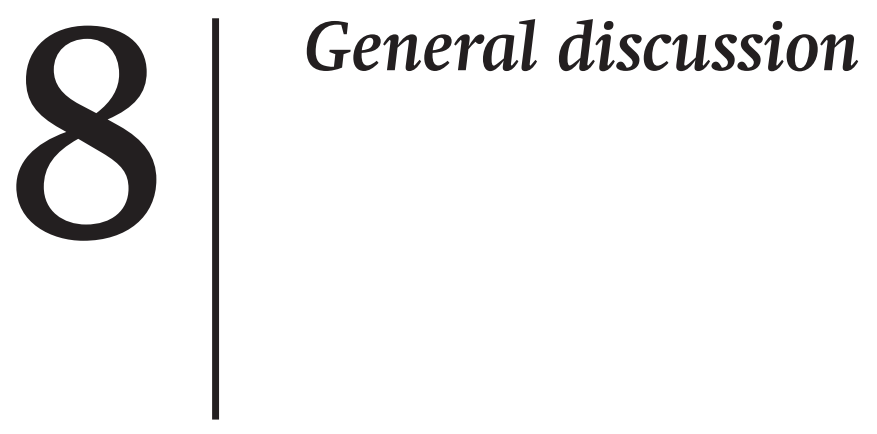



After the first positive results in the pioneering hospitals on the implementation of the enhanced recovery after surgery (ERAS) guideline in colorectal surgery [1], ERAS was implemented using a Breakthrough Series collaborative in a nationwide setting in colorectal surgery [2]. The sustainability of this implementation effort was evaluated subsequently [3, 4].

Following on these studies, this thesis focuses on the implementation of the ERAS guideline in the Netherlands. The acquired knowledge about implementation challenges contributed to the design of the studies performed within this thesis. It was aimed to provide further information on the process of implementation by exploring the adoption of the ERAS guideline in gynecologic surgery in the Netherlands. The challenge to define a preferred efficient strategy for the nationwide dissemination of best practices was addressed. By providing this evidence, the infrastructure of the randomized controlled trial was used to induce a practice change in Dutch hospitals at the same time. In this thesis the level of implementation was determined on process and outcome level.

This chapter presents an overview of the main findings and reflects on the context, process, and effectiveness of the nationwide implementation of ERAS in actual perioperative practice around gynecologic surgical procedures. The following research questions, addressed in chapter 1 , are summarized and discussed:

- Which ERAS elements are already used in gynecologic surgery and what is known about their effect on postoperative outcomes? (chapter 2)

- What is the current status of perioperative care in gynecologic surgery in the Netherlands and how is the adoption of ERAS elements in actual practice? (chapter 3)

- How do spontaneous diffusion and structured implementation of ERAS in other departments impact implementation of ERAS in gynecologic surgery and what is their relation to patient's outcomes? (chapter 3,4)

- Does an efficient, tailored model to implement ERAS across multiple hospitals make large scale implementation strategies more effective and to what degree do intervention fidelity and contextual factors influence implementation? (chapter $5,6,7)$

Methodological issues are discussed and future perspectives are described.

\section{SUMMARY OF MAIN FINDINGS}

The effect of the evidence-based ERAS guideline on patient outcomes in colorectal surgery was mainly investigated by performing pre-post analyses and randomized 
controlled trials. Evaluation of the literature on ERAS in gynecologic surgery showed that literature was limited. Included studies were mostly classified as case series (15 out of 16 studies). A large diversity in reported perioperative elements within studies was shown. Preoperative education, early oral intake, and early mobilization were the only elements included in all pathways. The length of hospital stay following an ERAS guideline ranged from 1 to 2 days among patients with a benign disease and from 5 to 7 days among patients with an oncologic indication for surgery. Five studies compared ERAS with traditional care and could be used for a quantitative analysis. A reduction of 1.6 days in primary length of hospital stay was observed after the adoption of an ERAS guideline. No significant differences were observed in complication and re-admission rates; however available data in the eligible studies were limited. Our review provided an overview of which single-evidence based elements were already used in gynecologic surgery. Acknowledging the limitations of the included studies, it was shown that implementation of ERAS in gynecologic surgery might be associated with a reduced length of postoperative hospital stay.

The adoption of the ERAS guideline in gynecologic surgery in the Netherlands was explored by performing two observational studies. Spread of ERAS principles to other surgical departments is possible, but occurred only partially in the Netherlands. A lack of progress with the implementation of ERAS was initially shown in the early adopter hospital, but findings proved to be transferrable to other hospitals as well. ERAS elements related to the preoperative and intra-operative phase were partially adopted in standard gynecologic practice, but adoption of the postoperative elements did not occur spontaneously after several years of practicing ERAS in colorectal surgery. Initial implementation effects seem to be restricted to the participating departments and do not automatically spread to other surgical teams in the same hospital. Even in the hospitals with a mixed ward for colorectal and gynecologic surgery patients, no adoption of the majority of ERAS elements occurred. The implication of these findings is that complete adoption of the evidence-based ERAS guideline in gynecology requires an active and organized implementation approach.

This provided the opportunity to combine the search for an optimal quality implementation strategy with the required improvement of perioperative practice in gynecologic surgery. An efficient evidence-based strategy to induce change on large scale level is still lacking. The challenge to define a preferred strategy for the nationwide dissemination of best practices was addressed in the study protocol. The ideal approach should be based on the existing evidence, could be adapted to varying clinical settings, and would be able to deliver an optimal effect at the lowest possible costs. The study protocol describes the framework of a tailored stepwise implementation approach comprising four levels of intensity of support. Theoretically, the stepped 
implementation strategy fulfilled the criteria for optimal implementation. Different change interventions, based on the Cochrane Effective Practice and Organization of Care (EPOC) database [5], were included. This newly designed strategy was compared with the traditional breakthrough strategy composing three generic educational sessions and the use of plan-do-study-act cycles for planning and executing local improvement activities. The cluster randomized controlled trial had a nationwide focus and a participation rate of $73 \%$ of all eligible hospitals was achieved. The data of 1098 patients treated in 14 hospitals were prospectively received and analyzed. Outcomes were compared with data received from a retrospective baseline audit of 420 patients treated in the same hospitals. Process data showed that fidelity to the implementation activities was poor in the tailored stepped implementation group. It was assumed that this was mainly due to the complexity of the design of the tailored stepped implementation strategy and the underestimation of this complexity and its time constraints. However, the stepped implementation strategy did not differ in effectiveness compared to the breakthrough strategy. Both strategies improved perioperative care and outcomes. An increase in compliance of over $30 \%$ in both study arms was reached. Based on the results of this randomized controlled trial, we were unable to define the preferred strategy for large scale implementation of clinical interventions. Both implementation strategies provided comparable results, however some major drawbacks existed, mainly related to time constraints in the design of the implementation strategy. Process analyses of the innovative tailored stepped implementation strategy showed several limitations that hinder actual use in daily practice. Further refinement and testing of this type of strategy is recommended. The design of a feasible tailored and stepped strategy, which could be more appropriate for use in daily practice, should be a topic for further research.

By exploring data of the nonparticipating hospitals, we provided information about the role of awareness in the process to change. Local stakeholders were initially approached to participate in a randomized controlled trial to implement the ERAS guideline in a research setting. These recruitment activities raised awareness that a nationwide change in perioperative care was intended. Our hypothesis was that this awareness could have induced a process to change local perioperative care. Data showed that these nonparticipating hospitals were able to adapt some of the ERAS elements, resulting in an improvement of evidence-based perioperative practice. The improvements however, were too limited to significantly change other outcome measures. Time to functional recovery and length of postoperative hospital stay remained unchanged. 


\section{METHODOLOGICAL CONSIDERATIONS}

\section{Study design}

The multicentre design of the studies strengthens conclusions and provides more generalizability of results. To our knowledge, the used designs were unique and provided new evidence in the field of knowledge transfer. In the randomized controlled trial, we focused on the process of change. The trial was designed to observe the implementation processes and effects in both study groups and was powered based on difference in effect between both strategies. We applied a Comparative Effectiveness Research (CER) trial [6], by using an active control group that was also exposed to an implementation strategy. Such CER trial is not often applied in existing implementation studies [7, 8]. Similarly to the Breakthrough Series collaborative [9], a multifaceted intervention was used in the tailored stepped implementation strategy. These interventions were mainly focused on changes at professionals' level. Although it is known that changes on organizational level should be included in the strategy as well $[10,11]$, we were unable to embed those changes structurally in clinical practice during the execution of the trial in all participating hospitals. The study followed an intention to treat analysis to prevent over estimation of the implementation effect and concealed allocation by an independent statistician was used to avoid bias. The time constraint was the major limitation for the tailored stepped implementation strategy. The 3-month evaluation periods were chosen to follow the timing of the breakthrough educational session. However, the time between audit and feedback was too short to plan an additional next step every three months if necessary. The poor fidelity in the tailored stepped implementation group could be considered as the most important limitation in the design of the innovative implementation strategy. Therefore, we decided not to perform the intended costminimization analysis as stated in the study protocol.

\section{Hospital selection}

Reflecting on Rogers' diffusion theory [12], it is probable that the included hospitals in the randomized controlled trial belong to the early adopter and early majority categories. The climate and willingness to change within these organizations may be more optimal compared to the hospitals that did not participate. This could have induced some selection bias. By providing information about the recruitment process, the nonparticipating organizations, and the contextual factors, a complete representation could be given which is better transferable to other settings. The participation rate of $73 \%$ of all eligible hospitals in the Netherlands helped to provide representative results as well. 


\section{Measurements}

Guideline adherence to key elements was evaluated and patient outcomes on length of recovery and length of hospital stay were measured throughout the different studies. As stated in the general introduction, ERAS guidelines consist of several interacting and evidence-based perioperative elements [13-15]. Several studies focusing on colorectal surgery have performed multivariate analysis, but results on the effect of individual elements were diverse [16-18]. In line with other studies, a selection of key elements was used as a measure for overall guideline adherence. This could have resulted in a restricted focus on the non-selected elements of the participating hospitals during the implementation process. The predefined elements could all be retrieved reliably and were assumed to directly influence functional recovery. Their combination should have reflected implementation of a complete ERAS pathway; however this assumption was not tested. Because of the synergistic nature of the elements, the effect of several of the unmeasured elements could have been greater than initially anticipated. The selection of key elements did ensure complete and correct recording of data. The data collection in all studies was carried out by one data abstractor, limiting bias in the data collection. Although this supported the collection of reliable data for the analysis, one data abstractor could have influenced implementation effects on the long term. With the cessation of the study, the total structure of data collection, analysis and feedback was ended. Precisely those elements of implementation are (audit and feedback) valuable for a sustained effect [4].

\section{Fidelity}

Fidelity to implementation activities was measured in the randomized controlled trial. Results showed that the fidelity in the breakthrough strategy group was acceptable. However, in the tailored stepped implementation group fidelity was poor and could be considered as one of the most important limitations in the design of the strategy. Although hospitals agreed that their actual care still deviated from ERAS, apparently they did not prioritize other learning sessions. In fact, only one hospital participated in the second and third implementation level. Professionals were unable to plan other educational meetings instead of performing clinical tasks in advance. The short period between the real-time feedback and meetings, made that they were unable to attend those meetings. In contrast, participants in the breakthrough strategy group acknowledged the additional effect of general learning sessions. Some professionals even attended those meetings beyond their working hours. 


\section{INTERPRETATION OF THE FINDINGS}

The effect of ERAS on patient outcomes was addressed in the systematic review. The available evidence, consisting of 16 observational studies, suggested that ERAS could reduce the length of postoperative hospital stay. Additional studies, performed afterwards, showed similar results and presented the positive influence of ERAS on patient satisfaction $[19,20]$, hospital costs [21, 22], and complication rates [23] within gynecologic surgery. Traditional care is often undefined which complicates thorough evaluation on the effect of ERAS pathways. The observed variation in the number and type of ERAS elements reveals a lack of standardization of the included elements. The consensus guideline of the ERAS Society in gynecologic surgery was published in 2016 [14, 15] and updated in 2019 [13]. No major differences in elements between both guidelines existed. With their evidence-based recommendations, an essential tool is provided to promote integration of existing knowledge into practice. During the last years more systematic reviews about ERAS in gynecologic surgery were published [24]. Although differences in design were acknowledged, conclusions about the positive role of ERAS in perioperative care are consistent.

In this thesis we had the unique opportunity to compare perioperative practice after gynecologic surgery in different settings and with a nationwide focus. The different settings were created by the previous implementation of ERAS in colorectal surgery. By using acquired knowledge and implementation theories, we were able to provide insight into the adoption and spread of ERAS elements. Spread can be defined as the process through which effective innovations are adopted from one setting to another. A continuum between unplanned (diffusion) and planned (dissemination or implementation) mechanisms are described. A retrospective study demonstrated that previous implementation of ERAS in colorectal surgery induced a positive impact on patients undergoing liver surgery on the same ward and treated by the same team [25]. In contrast, our results have shown that spread was limited between different departments. Apparently, barriers between different disciplines were even higher than initially expected.

During the last decades, knowledge about change management in health care has been improved. New evidence became available and existing concepts were adapted from other scientific fields. For instance, the diffusion theory of Rogers that divides a population in five main adopter categories was initially used in social sciences [12]. Several different implementation strategies have emerged and wellknown strategies are adapted constantly in an attempt to improve outcomes. With the development of initiatives to summarize the available evidence in frameworks and to concretize this in accessible guidelines for implementation, important steps were taken to further improve quality improvement processes [26, 27]. Despite 
these efforts, a gold standard for implementation of innovations is still lacking. There is an increasing need for an evidence based model to efficiently implement innovations on a large scale [26, 27]. Tailored interventions that take individual, social and organizational factors into account have shown some optimistic effects, but these interventions are more complex and difficult to apply at larger scale [28, 29]. Quality improvement interventions are often based on theoretical models. The cascade of change necessary to implement complex innovations can be categorized in several steps. Grol et al. [30, 31] developed a model consisting of five consecutive phases (awareness, understanding, acceptance, adoption, and integration of the proposed behavior). This stepwise framework was used in the design of the stepped implementation strategy. To our knowledge, this was the first study that used a stepped design combining tailored and successive implementation approaches. A final sustainability meeting was added in both models at the end of the implementation strategy. While it is recognized that the implementation of innovations in practice is challenging, achieved successes have to sustain after active implementation has ended. The long-term impact of implementation efforts was evaluated in a systematic literature review [32]. Given the limited number of studies at a high risk of bias, results suggest that adherence was not sustained in at least $30 \%$ of the evaluated implementation projects [32]. In the final sustainability meeting, tools were provided to sustain and improve the progressions made.

Recently, a systematic review of 53 studies was conducted to examine the factors that affected ERAS implementation [10]. The key facilitating factors included the possibility to adapt the pathway to local contexts, using a formal implementation framework, and leveraging supporters and fulltime ERAS coordinators [10]. Barriers of success included a resistance to change from health care professionals and a lack of resources for implementation [10]. Although it was not possible to correlate process data to the level of success, findings of our process analysis suggested similar results. The review showed that data about the process of implementation were limited and stated that more studies about implementation processes are needed to promote the spread of ERAS programs [10]. So, the findings of this thesis are a valuable addition to the existing evidence.

\section{IMPLICATIONS FOR PRACTICE}

Implementation of ERAS leads to improved perioperative outcomes and faster recovery after surgery. The potential implication of ERAS in clinical practice already proved to be far reaching. 
The process of recruiting hospitals for the implementation trial was slow and difficult, despite the use of targeted strategies and facilitators. Local stakeholders acknowledged the need for the nationwide standardization of perioperative practice along ERAS guideline, but some decided not to participate based on organizational and financial constraints. Our results proved that active implementation is necessary to change, which could help to convince organizations to participate in quality improvement projects to implement innovations in the future. The short-term constraints do not outweigh the long-term benefits. The high workload of specialists hampered participation. Therefore, increasing the role of other health care professionals in the process of change has to be considered as well. In the breakthrough strategy group, (specialized) nurses had a pivotal role during the implementation process. The national learning sessions were mainly visited by nurses who represented their hospitals and they leaded the local change. Traditionally, nursing practice was based more on habits and expertise than on evidence. Evidence-based nursing has gained interest during the last decades. The involvement of nurses in improvement programs, routine evidence-based nursing, and exchanging knowledge between nurses on different wards are important to improve practice.

Implementation of new evidence in clinical practice has to be improved. In this thesis we demonstrated the minimal spontaneous spread of innovations between surgical disciplines and between hospitals. In addition, the lack of an efficient evidence-based strategy for the nationwide dissemination of best practices was addressed. Based on our results, the design of a tailored stepped approach with increasing levels of support and the possibility to tailor activities to the individual needs of a specific organization seems to be promising. However, adaptations have to be made before this design will be appropriate for large scale implementation.

Besides improving implementation strategies, even more effort is needed to keep clinical practice up to date during the upcoming years. To improve evidence based care, changes in the structure of our health system and use of guidelines are also necessary. Several initiatives are already described in literature. The functional grouping of departments in Dutch hospitals seems to hamper spread of innovations and the multidisciplinary efforts to improve patient care. Knowledge sharing between different groups of healthcare professionals is restricted through group membership and can be considered as an intra-organizational barrier to change. The shift from a traditional function-oriented towards a process-oriented structure of hospitals has gained attention and could improve collaboration between different disciplines and facilitate spread of knowledge. Furthermore, involvement of knowledge brokers may promote spread of innovations across disciplines and hospitals [33]. Knowledge brokers are described as intermediaries to exchange knowledge across the varying stakeholders and settings. A recent review demonstrated that the 
availability of evidence about their effectiveness within healthcare is still limited so far [34].

At this moment, ERAS guidelines are almost completely implemented in gynecologic surgery in the Netherlands. In the hospitals that participated in the implementation trial some progress can be made. For the nonparticipating centers a local quality improvement program to update their perioperative practice conform the ERAS guideline seems indicated. Studies have shown that with increasing compliance to ERAS elements, even adverse events after surgery could be reduced in gynecologic surgery. We hope that the results presented in this thesis further stimulate professionals to take up the challenge to continuously improve their perioperative practice.

\section{IMPLICATION FOR FURTHER RESEARCH}

The results of the research presented in this thesis support further investigation in the field of large-scale implementation. There is sufficient evidence that supports the need for active implementation of innovations in clinical practice. However, more research is necessary to be able to define a preferred efficient strategy for the nationwide dissemination of best practices. Further refinement and testing of a stepped strategy, tailored to the individual needs of the participating organizations, seems to be recommended for large-scale implementation of best practices. The major drawback in our study was due the complexity in the design of the tailored stepped implementation strategy and its time constraints. By performing continuous audit of guideline adherence and implementation effects instead of a three-monthly feedback system, this delay could be minimized. In an ideal situation, real time feedback data within existing electronic health records should be available, to stimulate implementation effects, ensure sustainability, and facilitate research. To optimize the effect of quality improvement efforts, further research is required to provide potential solutions to promote knowledge sharing within and between hospital walls and to extend the initial positive effects of quality improvement projects to different settings.

Organizations voluntarily participate in research initiatives. This can lead to non-representative effect sizes of implementation collaboratives. It has been shown that public health organizations involved in research networks are more engaged in implementation activities [35]. Research on the effect of implementation strategies should therefore also include additional information about the nonparticipating organizations. 


\section{CONCLUSION}

This thesis highlights the total process of change and demonstrates how an evidencebased innovation is implemented in daily practice. It shows that awareness raised by recruitment activities and successful implementation on other departments alone is not enough to facilitate change in patient outcomes. The results emphasize the challenge to spread and actively implement ERAS in actual practice. So, investments in structured implementation programs are necessary. No differences in the positive effect between the tailored stepped strategy and the traditional breakthrough strategy were found. The design of a stepped approach with increasing levels of support and the possibility to tailor activities to the individual needs of a specific organization is promising. However, adaptations have to be made before this design will be appropriate for large scale implementation. Our findings support the design of other implementation studies and help to better understand the process of change. 


\section{REFERENCES}

1. Maessen J, Dejong CH, Hausel J, Nygren J, Lassen K, Andersen J, Kessels AG, Revhaug A, Kehlet $\mathrm{H}$, Ljungqvist $\mathrm{O}$ et al: A protocol is not enough to implement an enhanced recovery programme for colorectal resection. Br J Surg 2007, 94(2):224-231.

2. Gillissen F, Hoff C, Maessen JM, Winkens B, Teeuwen JH, von Meyenfeldt MF, Dejong CH: Structured synchronous implementation of an enhanced recovery program in elective colonic surgery in 33 hospitals in The Netherlands. World J Surg 2013, 37(5):1082-1093.

3. Gillissen F, Ament SM, Maessen JM, Dejong CH, Dirksen CD, van der Weijden T, von Meyenfeldt MF: Sustainability of an enhanced recovery after surgery program (ERAS) in colonic surgery. World J Surg 2015, 39(2):526-533.

4. Ament SMC, Gillissen F, Moser A, Maessen JMC, Dirksen CD, von Meyenfeldt MF, van der Weijden T: Factors associated with sustainability of 2 quality improvement programs after achieving early implementation success. A qualitative case study. Journal of evaluation in clinical practice 2017, 23(6):1135-1143.

5. Cochrane Effective Practice and Organisation of Care Review Group: Data Collection Checklist. Ontario: Institute of Population Health. 2011, University of Ottawa. In.

6. Sox HC, Goodman SN: The methods of comparative effectiveness research. Annual review of public health 2012, 33:425-445.

7. Davies P, Walker AE, Grimshaw JM: A systematic review of the use of theory in the design of guideline dissemination and implementation strategies and interpretation of the results of rigorous evaluations. Implement Sci 2010, 5:14.

8. Goodacre S: Uncontrolled before-after studies: discouraged by Cochrane and the EMJ. Emergency medicine journal : EMJ 2015, 32(7):507-508.

9. Kilo CM: A framework for collaborative improvement: lessons from the Institute for Healthcare Improvement's Breakthrough Series. Quality management in health care 1998, 6(4):1-13.

10. Stone AB, Yuan CT, Rosen MA, Grant MC, Benishek LE, Hanahan E, Lubomski LH, Ko C, Wick EC: Barriers to and Facilitators of Implementing Enhanced Recovery Pathways Using an Implementation Framework: A Systematic Review. JAMA surgery 2018, 153(3):270-279.

11. Francis NK, Walker T, Carter F, Hubner M, Balfour A, Jakobsen DH, Burch J, Wasylak T, Demartines N, Lobo DN et al: Consensus on Training and Implementation of Enhanced Recovery After Surgery: A Delphi Study. World J Surg 2018, 42(7):1919-1928.

12. Rogers E. Diffusion of Innovations. 5th ed. New York: Free Press: 2003.

13. Nelson G, Bakkum-Gamez J, Kalogera E, Glaser G, Altman A, Meyer LA, Taylor JS, Iniesta M, Lasala J, Mena G et al: Guidelines for perioperative care in gynecologic/oncology: Enhanced Recovery After Surgery (ERAS) Society recommendations-2019 update. Int J Gynecol Cancer 2019, 29(4):651-668.

14. Nelson G, Altman AD, Nick A, Meyer LA, Ramirez PT, Achtari C, Antrobus J, Huang J, Scott M, Wijk L et al: Guidelines for postoperative care in gynecologic/oncology surgery: Enhanced Recovery After Surgery (ERAS(R)) Society recommendations--Part II. Gynecol Oncol 2016, 140(2):323-332.

15. Nelson G, Altman AD, Nick A, Meyer LA, Ramirez PT, Achtari C, Antrobus J, Huang J, Scott M, Wijk L et al: Guidelines for pre- and intra-operative care in gynecologic/oncol- 
ogy surgery: Enhanced Recovery After Surgery (ERAS(R)) Society recommendations--Part I. Gynecol Oncol 2016, 140(2):313-322.

16. Aarts MA, Okrainec A, Glicksman A, Pearsall E, Victor JC, McLeod RS: Adoption of enhanced recovery after surgery (ERAS) strategies for colorectal surgery at academic teaching hospitals and impact on total length of hospital stay. Surg Endosc 2012, 26(2):442-450.

17. Feroci F, Lenzi E, Baraghini M, Garzi A, Vannucchi A, Cantafio S, Scatizzi M: Fast-track Surgery in Real Life: How Patient Factors Influence Outcomes and Compliance With an Enhanced Recovery Clinical Pathway After Colorectal Surgery. Surg Laparosc Endosc Percutan Tech, 23(3):259-265.

18. Simpson JC, Moonesinghe SR, Grocott MP, Kuper M, McMeeking A, Oliver CM, Galsworthy MJ, Mythen MG, National Enhanced Recovery Partnership Advisory B: Enhanced recovery from surgery in the UK: an audit of the enhanced recovery partnership programme 2009-2012. Br J Anaesth 2015, 115(4):560-568.

19. Lee J, Asher V, Nair A, White V, Brocklehurst C, Traves M, Bali A: Comparing the experience of enhanced recovery programme for gynaecological patients undergoing laparoscopic versus open gynaecological surgery: a prospective study. Perioperative Medicine 2018, 7(1):15.

20. Philp S, Carter J, Pather S, Barnett C, D’Abrew N, White K: Patients' satisfaction with fast-track surgery in gynaecological oncology. European journal of cancer care 2015, 24(4):567-573.

21. Pache B, Joliat G-R, Hübner M, Grass F, Demartines N, Mathevet P, Achtari C: Costanalysis of Enhanced Recovery After Surgery (ERAS) program in gynecologic surgery. Gynecologic Oncology 2019, 154(2):388-393.

22. Gerardi MA, Santillan A, Meisner B, Zahurak ML, Diaz Montes TP, Giuntoli RL, 2nd, Bristow RE: A clinical pathway for patients undergoing primary cytoreductive surgery with rectosigmoid colectomy for advanced ovarian and primary peritoneal cancers. Gynecol Oncol 2008, 108(2):282-286.

23. Kalogera E, Bakkum-Gamez JN, Jankowski CJ, Trabuco E, Lovely JK, Dhanorker S, Grubbs PL, Weaver AL, Haas LR, Borah BJ et al: Enhanced recovery in gynecologic surgery. Obstet Gynecol 2013, 122(2 Pt 1):319-328.

24. Trowbridge ER, Dreisbach CN, Sarosiek BM, Dunbar CP, Evans SL, Hahn LA, Hullfish KL: Review of enhanced recovery programs in benign gynecologic surgery. International urogynecology journal 2018, 29(1):3-11.

25. Labgaa I, Jarrar G, Joliat GR, Allemann P, Gander S, Blanc C, Hubner M, Demartines N: Implementation of Enhanced Recovery (ERAS) in Colorectal Surgery Has a Positive Impact on Non-ERAS Liver Surgery Patients. World J Surg 2016, 40(5):1082-1091.

26. Gagliardi AR, Alhabib S, members of Guidelines International Network Implementation Working G: Trends in guideline implementation: a scoping systematic review. Implement Sci 2015, 10:54.

27. Grimshaw JM, Eccles MP, Lavis JN, Hill SJ, Squires JE: Knowledge translation of research findings. Implement Sci 2012, 7:50.

28. Baker R, Camosso-Stefinovic J, Gillies C, Shaw EJ, Cheater F, Flottorp S, Robertson N: Tailored interventions to overcome identified barriers to change: effects on professional practice and health care outcomes. Cochrane Database Syst Rev 2010(3):CD005470. 
29. Wensing M, Oxman A, Baker R, Godycki-Cwirko M, Flottorp S, Szecsenyi J, Grimshaw J, Eccles M: Tailored Implementation For Chronic Diseases (TICD): a project protocol. Implement Sci 2011, 6:103.

30. Grol R, Wensing M: What drives change? Barriers to and incentives for achieving evidence-based practice. Med J Aust 2004, 180(6 Suppl):S57-60.

31. Grol R: Implementing guidelines in general practice care. Qual Health Care 1992, 1(3):184-191.

32. Ament SMC, de Groot JJA, Maessen JMC, Dirksen CD, van der Weijden T, Kleijnen J: Sustainability of professionals' adherence to clinical practice guidelines in medical care: a systematic review. BMJ Open 2015, 5(12):e008073.

33. Waring J, Currie G, Crompton A, Bishop S: An exploratory study of knowledge brokering in hospital settings: facilitating knowledge sharing and learning for patient safety? Social science \& medicine 2013, 98:79-86.

34. Bornbaum CC, Kornas K, Peirson L, Rosella LC: Exploring the function and effectiveness of knowledge brokers as facilitators of knowledge translation in health-related settings: a systematic review and thematic analysis. Implement Sci 2015, 10:162.

35. Wilson T, Berwick DM, Cleary PD: What Do Collaborative Improvement Projects Do? Experience from Seven Countries. The Joint Commission Journal on Quality and Safety 2003, 29(2):85-93. 



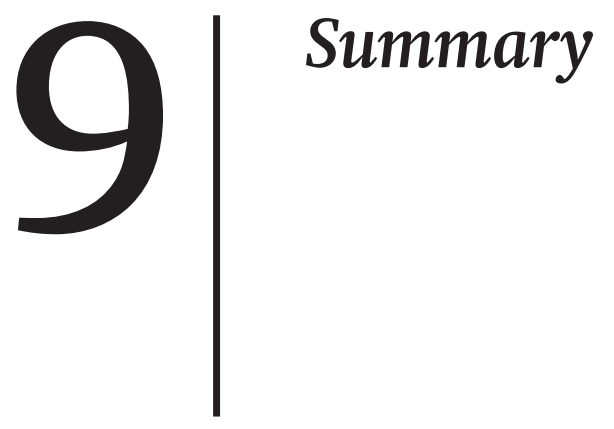



This chapter summarizes the findings of the research performed in this thesis and highlights the most important conclusions.

Chapter 1 describes the challenges of using evidence-based medicine in daily practice and focuses on knowledge translation and multi-organizational change. Recent developments in perioperative care in the light of the Enhanced Recovery After Surgery (ERAS) program are reported. Besides, the outline of this thesis and related research questions are presented in this chapter.

In chapter 2, a systematic search and review to identify current evidence of ERAS in gynecologic surgery was performed. The search resulted in the inclusion of 16 observational studies at high risk of bias, of which five studies were eligible for quantitative analysis. A large diversity in reported perioperative elements between studies was shown. Preoperative education, early oral intake, and early mobilization were the only elements included in all pathways. The length of hospital stay after ERAS implementation ranged from 1 to 2 days among patients with a benign disease and from 5 to 7 days among patients with an oncologic indication for surgery. Based on the meta-analysis, we suggested that implementation of ERAS elements might result in a reduced length of hospital stay (mean difference 1.6 days, 95\% CI -2.9 to -0.2) after abdominal gynecologic surgery.

Chapter 3 focuses on the spread of ERAS elements between surgical departments. The hypothesis that positive experiences and implementation tools gained after running a quality improvement project would stimulate spread to another closely related intra-organizational department was tested. ERAS elements related to the preoperative and intra-operative phase were partially adopted in standard gynecologic practice, but adoption of the postoperative elements did not occur spontaneously after several years of practicing ERAS in colorectal surgery. This multicenter observational study suggested that interdepartmental spread of universal evidence-based innovations within organizations was restricted. Multilevel regression analysis of 684 patients showed that perioperative care and outcomes did not differ between the gynecology departments of hospitals that previously followed a quality improvement project for colorectal surgery and the hospitals that did not.

In chapter 4 the impact of implementation activities to increase adherence to ERAS elements was investigated. An audit of 77 patients undergoing gynecologic cancer surgery was performed in an early adopter hospital. Theoretically, this center should have the optimal characteristics to change daily practice. Outcomes were compared with a historical cohort of 38 patients treated before structured implementation of ERAS. A reduced time to functional recovery (median 3 versus 6 days) and a shorter length of hospital stay (median 5 versus 7 days) were achieved. This chapter shows that implementation activities are still necessary and effective to improve perioperative practice. 
The opportunity to combine the search for an optimal quality implementation strategy with the required improvement of perioperative practice in gynecologic surgery was used to design a multicentre, cluster-randomized controlled trial. Chapter 5 presents the study protocol and describes the characteristics and framework of two different implementation strategies to change nationwide perioperative care in gynecologic surgery. The objective is to compare a tailored stepped approach that is characterized by tailoring the intensity of implementation activities to the needs of organizations and local barriers for change with a generic breakthrough strategy that is usually applied in large-scale improvement projects. The methodology to evaluate both implementation strategies is presented.

Chapter 6 reports the results of a multicentre, cluster-randomized controlled trial performed in 14 Dutch gynecologic oncology departments. A total of 1048 patients underwent surgery during the one-year period to implement ERAS in perioperative care. Analysis at three to 12 months after the start of implementation activities, revealed a significant increase in guideline adherence in both the breakthrough and tailored stepped implementation strategy. Mixed-effect regression models did not reveal any significant differences in effect between study groups in postoperative length of hospital stay (mean difference (MD) -0.5 days; 95\% CI -1.9 to 0.9; $\mathrm{p}=$ 0.476 ), length of recovery (MD -0.36 days; $95 \% \mathrm{CI}-1.47$ to $0.76 ; \mathrm{p}=0.497$ ); and overall guideline adherence (MD -3.0\%; 95\% CI -13.6 to 7.6; $\mathrm{p}=0.583$ ). Process analyses of the tailored stepped implementation strategy showed several limitations that hinder actual use in daily practice. Fidelity to the implementation activities was poor and further refinement and testing of this type of strategy is recommended.

In chapter 7, it was shown that neither hospital characteristics nor perioperative outcomes at baseline differed between the participating and nonparticipating departments of a national large-scale implementation project. In addition, we explored how perioperative practice changed among nonparticipants. Linear regression models revealed no statistically significant or clinically relevant differences in time to functional recovery or length of hospital stay. Despite its exploratory nature, this study offers some insight into the influence of large-scale implementation projects on clinical practice in nonparticipating hospitals.

Chapter 8 presents a general discussion of findings addressed in this thesis within the context of the recent literature. Subsequently, a reflection on methodological considerations, practical implications, and future perspectives were provided. 




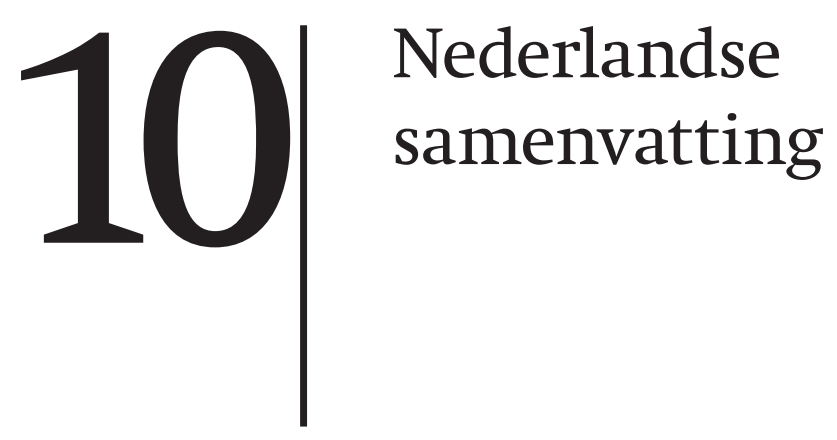



Dit hoofdstuk beschrijft de bevindingen van het onderzoek uitgevoerd binnen deze thesis en belicht de meest belangrijke conclusies die getrokken kunnen worden.

Hoofdstuk 1 geeft de uitdagingen die gepaard gaan met het gebruik van evidencebased medicine in de dagelijkse praktijk weer. Dit hoofdstuk focust zich op de toepassing van eerder verworven kennis en op verandering binnen meerdere organisaties. Recente ontwikkelingen binnen de perioperatieve zorg met betrekking tot het Enhanced Recovery After Surgery (ERAS) programma worden besproken. Daarnaast wordt er een overzicht gegeven over de inhoud van deze thesis en gerelateerde onderzoeksvragen.

Hoofdstuk 2 beschrijft de uitkomsten van een systematische zoekstrategie en review over de beschikbare literatuur over ERAS binnen de gynaecologische chirurgie. Deze zoekstrategie resulteerde in de inclusie van 16 observationele studies met een hoog risico op bias. Vijf van deze studies waren geschikt voor een kwantitatieve analyse. Er bestond een grote diversiteit in gerapporteerde perioperatieve elementen tussen studies. Preoperatieve educatie, snel hervatten van orale intake en vroege mobilisatie na de ingreep waren de enige elementen die in alle studies werden geïncludeerd. De opnameduur na implementatie van ERAS was een tot twee dagen bij patiënten met een benigne indicatie en vijf tot zeven dagen bij patiënten met een oncologische indicatie voor de chirurgische ingreep. Gebaseerd op de resultaten van de meta-analyse, suggereren we dat de implementatie van ERAS kan resulteren in een kortere opnameduur na laparotomische gynaecologische chirurgie (verschil 1.6 dagen, 95\% betrouwbaarheidsinterval -2.9 tot -0.2 ).

Hoofdstuk 3 focust zich op de verspreiding van ERAS elementen tussen chirurgische afdelingen. De hypothese dat de positieve ervaringen en implementatie vaardigheden verworven bij het uitvoeren van een kwaliteitsverbeteringsprogramma de verspreiding naar andere afdelingen binnen dezelfde organisatie zou stimuleren, werd getoetst. ERAS elementen gerelateerd aan de pre-operatieve en intra-operatieve fase werden gedeeltelijk toegepast in de standaard gynaecologische zorg. Adoptie van de postoperatieve elementen heeft, na het jarenlang uitvoeren van ERAS in de colorectale chirurgie, nog niet spontaan plaatsgevonden. Deze multicenter observationele studie suggereert dat de verspreiding van universele evidence-based innovaties tussen afdelingen binnen dezelfde organisatie beperkt is. Multilevel regressie analyses van 684 patiënten lieten zien dat de perioperatieve zorg en uitkomsten niet verschillen tussen de gynaecologische afdelingen van ziekenhuizen die eerder een kwaliteitsverbeteringsprogramma binnen de colorectale chirurgie hebben uitgevoerd en de ziekenhuizen die dat niet deden.

In hoofdstuk 4 wordt de impact van implementatie activiteiten op de toename in adherentie van ERAS elementen onderzocht. Een audit van 77 patiënten met een gynaecologische maligniteit die een operatieve behandeling kregen, werd uitgevoerd 
in een early adopter ziekenhuis. Theoretisch gezien zou dit centrum de optimale karakteristieken moeten hebben om de dagelijkse praktijk te veranderen. Uitkomsten werden vergeleken met een cohort van 38 patiënten die behandeld werden voordat ERAS gestructureerd werd ingevoerd. Een reductie in herstelduur (mediaan 3 versus 6 dagen) en een kortere opnameduur (mediaan 5 versus 7 dagen) werden bereikt. Dit hoofdstuk laat zien dat implementatie activiteiten nog steeds nodig en effectief zijn om de perioperatieve zorg te verbeteren.

De zoektocht naar een optimale strategie voor kwaliteitsverbetering, gecombineerd met de noodzakelijke verbetering in perioperatieve zorg binnen de gynaecologische chirurgie, gaf de mogelijkheid om een multicenter, cluster-gerandomiseerde trial uit te voeren. Hoofdstuk 5 toont het studieprotocol en beschrijft de karakteristieken en het framework van twee verschillende implementatie strategieën om de landelijke perioperatieve zorg binnen de gynaecologische chirurgie te veranderen. Het doel is om een stapsgewijze strategie op maat te vergelijken met de algemene doorbraak strategie welke meestal wordt toegepast in verbetertrajecten op grote schaal. De stapsgewijze strategie wordt gekenmerkt doordat de intensiteit van implementatie activiteiten is aan te passen aan de behoeftes van de organisatie en aan de lokale belemmeringen voor verandering. De methodologie om beide implementatie strategieën te evalueren wordt weergegeven.

Hoofdstuk 6 bespreekt de resultaten van een multicenter, cluster gerandomiseerde trial die werd uitgevoerd binnen 14 gynaecologische oncologie afdelingen in Nederland. Een totaal van 1048 patiënten onderging een operatie gedurende de periode van 1 jaar om ERAS in de perioperatieve zorg te implementeren. Analyses op 3 tot 12 maanden na start van de activiteiten lieten een significante toename zien in richtlijnopvolging in zowel de doorbraak als de stapsgewijze implementatie strategie. Mixed-effect regressie modellen lieten geen significante verschillen zien tussen de studiegroepen in opnameduur (gemiddeld verschil -0.5 dagen; 95\% betrouwbaarheidsinterval -1.9 tot $0.9 ; \mathrm{p}=0.476$ ), herstelduur (gemiddeld verschil -0.36 dagen; $95 \%$ betrouwbaarheidsinterval -1.47 tot 0.76 ; $\mathrm{p}=0.497$ ); en richtlijnopvolging (gemiddeld verschil $-3.0 \%$; 95\% betrouwbaarheidsinterval -13.6 tot 7.6 ; $\mathrm{p}=0.523$ ). Procesanalyse van de stapsgewijze implementatie strategie liet verschillende beperkingen zien voor het daadwerkelijk gebruik in de dagelijkse praktijk. Deelname aan implementatie activiteiten was beperkt en verdere aanpassing en controle van dit type strategie wordt aanbevolen.

In hoofdstuk 7 werd aangetoond dat er geen verschil is in de kenmerken per ziekenhuis en de perioperatieve uitkomsten op baseline tussen de afdelingen die wel en niet deelnemen aan een landelijk implementatie project. Daarnaast werd de verandering in perioperatieve zorg geëvalueerd binnen de niet deelnemende ziekenhuizen. Lineaire regressie modellen lieten zowel geen statistisch als klinisch 
relevant verschil zien in herstel- en opnameduur. Ondanks de exploratieve insteek, geeft deze studie inzicht in de invloed op de klinische praktijk van implementatieprojecten op grote schaal in niet deelnemende ziekenhuizen.

Hoofdstuk 8 presenteert een algemene discussie van de bevindingen die in deze thesis worden besproken in de context van de recente literatuur. Daaropvolgend wordt er een reflectie op de methodologische overwegingen, praktische implicaties en toekomstige perspectieven gegeven. 

$\left.11\right|^{\text {Epilogue }}$ 



\section{VALORIZATION}

This chapter discusses the valorization potential of the research presented in this thesis. The relevance and implications on professional, societal, and economic perspective were already addressed in the introduction and discussion in this thesis and throughout the individual chapters. In this paragraph we will shortly summarize the implications of the main findings. The challenge of the implementation of the best available evidence in daily practice, with a focus on perioperative care after gynecologic surgery in the Netherlands, is the focus of this thesis.

Since the first successful report of a multimodal program for colorectal surgery by Kehlet in 1997, the use of perioperative care programs has increased significantly worldwide. In 2001, the ERAS Society was initiated by a group of early adopters in Europe to enhance recovery and reduce complications after surgery. The multidisciplinary and multimodal approach was based on existing evidence and includes all available elements of care that improve recovery. Structured implementation (compliance of $70 \%$ or more) results in a reduction in hospital stay, a reduction in postoperative complications, and a reduction in overall health care costs. So, the concept of ERAS could be considered as an important example of value-based care. The collaborative promoted the development and spread of perioperative care protocols, but also focused on education and implementation of best practices. The Netherlands was one of these early adopters. ERAS was implemented successfully in colorectal surgery, between 2005 and 2009, by using a Breakthrough Series model in 33 Dutch hospitals. Our review showed that the multimodal elements could be applied to gynecologic surgery as well, with similar positive results. The nationwide implementation of ERAS in gynecologic surgery was evaluated in this thesis. Implementation of ERAS contributes to the standardization of perioperative practice, which is essential to improve health care. However, still variation exists in perioperative care elements between different hospitals. The (prolonged) use of thrombosis prophylaxis and different pain treatment strategies are, for example, elements with a lack of consistent evidence and consensus. Audit of process measures and patient outcomes are necessary to reveal those differences in clinical care. Besides fundamental research generating improved medical knowledge, health care research generating insight into clinical practice variation is necessary to compare and spread best practices in health care.

The concept of valorization is based on the underlying value of knowledge. Structured implementation of this knowledge in daily practice is essential. The implementation of quality improvement initiatives on large scale proved to be challenging and research on how to optimize resources is warranted. Although this thesis was focused on an innovation in gynecologic surgery, results about the presented 
implementation challenges are relevant for a broader audience. The results were published in peer reviewed international journals and were presented at national and international scientific conferences. During the research project, we had a nationwide focus and multidisciplinary health care professionals were involved. The patient association for gynecologic cancer (OLJF) and the national collaborative of professionals involved in the treatment of women with gynecologic cancer (DGOG) were contacted before the start of the implementation project. The DGOG supported the underlying aim to reach nationwide standardization of perioperative care consistent with the ERAS program. This stimulated awareness at professional, hospital, and patient level. As part of the implementation process actual care was measured, evaluated, and improved. Although these results are promising, still progress can be made to refine the findings described in this thesis, and to stimulate the development of more efficient and sustainable large-scale implementation programs in the future. 


\section{CONTRIBUTING CENTERS}

Academic Medical Centre, Amsterdam

I. Burke, G. Fons, F. Kosterman

Albert Schweitzer Hospital, Dordrecht

L.N. Hofman

Amphia Hospital, Breda

D. van Hamont, A.L.M. Kok

Bravis Hospital, Bergen op Zoom

J. Schellekens, P.M.L.H. Vencken

Canisius Wilhelmina Hospital, Nijmegen

M.P.L.M. Snijders

Catharina Hospital, Eindhoven

Y. Boomgaard, R.H.M. Hermans, L. Jue, R. Tops, I. Vandenput, E. van de Ven

Diakonessenhuis, Utrecht

M. van Haaften

Elisabeth-TweeSteden Hospital, Tilburg

C. Diepenhorst, N. van Leijsen, J.M.A. Pijnenborg, K. Pouls, M. van Vroenhoven

Erasmus Medical Centre, Rotterdam

S. Blom, F. Grune, L. Holley, E.M. Roes, W. Visser

Franciscus Hospital, Rotterdam

W. Hofhuis

HagaZiekenhuis, The Hague

K. van Dijk, A.M.L.D. van Haaften-de Jong, M. van der Hel, M. Rijsbergen, S. Verheule Isala Klinieken, Zwolle

H.H. de Haan

Jeroen Bosch Hospital,'s Hertogenbosch

B.M. Pijlman

Leiden University Medical Centre, Leiden

K.N. Gaarenstroom, M. Hogendorf, M. Löwik, M. Westerink

Maasstad Hospital, Rotterdam

J.E. Martens

Maastricht University Medical Centre, Maastricht

J. Goedmakers, M. Kamps, C. Penders, M. van de Voort

Maxima Medical Centre, Veldhoven

E.A. Boss

Meander Medical Centre, Amersfoort

C.G. Gerestein, A. Krol

Medisch Spectrum Twente, Enschede 
Y. Bredenhoff, M. Otto, N. Reesink-Peters, S. Arendsen

Netherlands Cancer Institute, Amsterdam

W.J. van Driel

Radboud University Nijmegen Medical Centre, Nijmegen

R.L.M. Bekkers

St Antonius Hospital, Nieuwegein

J.H. Schagen van Leeuwen, M. van Nieuwaal

University Medical Centre Utrecht, Utrecht

E.B.L. van Dorst

Zuyderland Hospital, Sittard-Geleen

H. Mertens 


\section{DANKWOORD}

Tot slot het dankwoord, een moment waarbij ik mijn dank voor alle begeleiding, ondersteuning, vertrouwen en motivatie die ik mocht ontvangen gedurende mijn promotietraject op papier kan zetten. Ik snap wel waarom men spreekt over een traject, want dat is het zeker geweest. De grootste stappen zijn al enige tijd geleden gezet en diverse mensen hebben hier direct en indirect aan bijgedragen. Wellicht vergeet ik iemand, maar de volgende personen wil ik graag in het bijzonder bedanken:

Bovenal mijn promotieteam, prof. dr. van der Weijden, dr. Slangen en dr. Maessen. Voor mij was dit onderzoek een schot in de roos: praktisch, klinisch toepasbaar en ook nog eens binnen de gynaecologische oncologie. Vanaf het begin heb ik hier met heel veel plezier aan gewerkt. Ondanks dat dit de laatste periode met wat horten en stoten ging, kijk ik met trots terug op wat we bereikt hebben. Trudy, dank voor de mogelijkheid die je me hebt gegeven om te promoveren op dit implementatieproject. Je heb me vrij gelaten in het verder uitwerken hiervan, waarbij er alle ruimte was voor nieuwe ideeën en onderzoeksvragen. Met je visie, ervaring en vertrouwen zijn we in staat geweest om daadwerkelijk veranderingen door te voeren. Ik wil je bedanken voor de fijne samenwerking, je inspiratie en je vertrouwen in mij tot en met de laatste loodjes. Beste Brigitte, jij was een onmisbare schakel. Met je kundigheid, ervaring, inzet en hulp heb je me de juiste weg op geholpen. Tijdens al je verplichtingen in de kliniek wist je altijd wel tijd voor mij vrij te maken, iets wat $\mathrm{ik}$, met name nu, alleen maar enorm kan bewonderen. Bedankt voor je begeleiding, ik heb veel van je geleerd, tijdens mijn onderzoekstijd, maar bovenal in de kliniek. Je nieuwe nevenfunctie als plaatsvervangend opleider binnen het cluster komt dan ook niet als een verassing. José, jij was de spil in het geheel en wist de theorie en praktijk moeiteloos te verbinden. Tijdens mijn promotietraject wist je me altijd de juiste kant op te sturen. Je oprechte interesse in mij als persoon en je adviezen als copromoter, collega, maar soms ook als moeder, heb ik altijd gewaardeerd. Dank voor al je hulp en toewijding tijdens mijn promotietraject.

Prof. Dirksen, prof. Dejong en prof. Kruitwagen, ondanks dat jullie niet tot het officiële promotieteam horen, beschouw ik jullie wel als onderdeel van het team. Ik waardeer jullie enthousiasme, vriendelijkheid en behulpzaamheid. Carmen, je sloot altijd aan bij alle besprekingen en je feedback heeft zeker bijgedragen aan de successen. Gezien de uitkomsten van de trial is de kosteneffectiviteitsanalyse er helaas nooit definitief van gekomen. Prof. Dejong, dank voor uw adviezen en waardevolle feedback. Prof. Kruitwagen, vanaf de zijlijn was u altijd betrokken bij het project en was $u$ altijd bereid om mee te denken. Dank voor alle support, als persoon en als opleider. 
Prof. dr. Faber, dr. van Bodegom - Vos, prof. dr. van Gorp, prof. dr. de Hingh en prof. dr. van Merode, ik wil u graag bedanken dat $\mathrm{u}$ deel wilde uitmaken van de manuscript commissie en tevens voor de tijd en moeite die daarmee gepaard gaat.

Voor het daadwerkelijke implementatietraject zijn alle deelnemende centra onmisbaar geweest. Dank voor jullie deelname aan de bijeenkomsten en jullie effort om ERAS in de dagelijkse praktijk te implementeren. Door jullie bereidheid tot verandering hebben we een belangrijke bijdrage kunnen leveren in het verbeteren van de perioperatieve zorg. Het heeft me heel wat treinuren gekost, maar ik werd elke keer hartelijk ontvangen als ik weer langskwam voor de dataverzameling. Speciaal dank aan de contactpersonen en de lokale implementatieteams, zonder jullie was het nooit zover gekomen. Een speciaal woord van dank voor Loes Schouten. Destijds als senior adviseur van het $\mathrm{CBO}$, heb je een grote bijdrage gehad bij het uitvoeren van de doorbraakstrategie. We mochten blij zijn dat jij, als expert in het invoeren en verspreiden van innovaties, betrokken wilde zijn bij dit traject.

Ook dank aan mijn medeauteurs voor hun bijdrage. Bjorn Winkens, dank voor je hulp bij het opzetten van de statistische analyses. Maite, bedankt voor al je werk bij de dataverzameling voor de nonparticipant ziekenhuizen. Inmiddels heb je zelf al een mooie promotie achter de rug. Alle andere medeauteurs wil ik graag danken voor hun kritische blik, inbreng en betrokkenheid.

Beste Stephanie en Iris, tijdens mijn onderzoekstijd waren jullie een welkome afleiding, dat heeft de (statistiek)curssussen toch wat luchtiger gemaakt. Stephanie, een van die cursussen was de aanzet voor twee mooie reviews. Dank voor al je hulp.

Vanzelfsprekend wil ik ook alle mensen binnen de vakgroep huisartsgeneeskunde bedanken. Jullie hebben mij een goede werkplek geboden tijdens mijn onderzoek en ik heb veel kunnnen leren van jullie onderzoekstrajecten en visie.

Petra en Karin, jullie zijn als secretariële ondersteuning onmisbaar. Dank voor jullie hulp bij het inplannen van alle afspraken en voor alle regelzaken. Charlotte en Moniek, jullie waren door al jullie inzet voor de implementatie van ERAS binnen het MUMC+ echte experts. Dank dat jullie een landelijk aanspreekpunt wilden zijn voor alle verpleegkundig specialisten.

Beste stafleden gynaecologie van het MUMC+ en het Zuyderland MC, jullie hebben allemaal op je eigen manier een belangrijke rol in het fijne opleidingsklimaat. Dank voor jullie interesse in mijn promotietraject en jullie investering in mijn ontwikkeling als arts en als persoon. Martine en Nicol, jullie hebben de kwaliteit van de opleiding binnen het Zuyderland naar een hoger niveau weten te tillen, uiteraard met steun van de hele vakgroep. Ik kijk uit naar alle mooie en leerzame momenten die nog komen gaan. Ook alle andere collega's op de verloskamers, het secretariaat, de polikliniek en verpleegafdeling wil ik hartelijk bedanken voor de fijne samenwerking. 
Beste collega arts-assistenten, dank voor de mooie opleidingstijd die we samen hebben. De gezelligheid met elkaar, maar ook steun voor elkaar is onmisbaar. Een speciaal woord van dank voor mijn (semi)jaargenootjes en carpoolmaatjes. Onze discussies, verhalen, successen en blunders werken verhelderend, ontnuchterend en relativerend. Wat ben ik blij met jullie!

Natuurlijk wil ik ook mijn vriendengroep bedanken. Vijftien jaar geleden leerden we elkaar kennen bij de start van de opleiding en inmiddels is er een hechte vriendschap ontstaan. Ik ben enorm trots op wat jullie in die tijd bereikt hebben. Carmen, Daphne, Emmi, Lieve, Saman, Sophie, Tim en partners, bedankt voor jullie onvoorwaardelijke vriendschap en steun, ook in roerige tijden. Maar vooral voor alle ontspanning buiten het werken om. Carmen, op bepaalde fronten zijn we heel verschillend, maar we hebben dezelfde werkethos en streven beiden naar perfectie. Dank voor je bemoedigende woorden en dat je ook vandaag naast me staat.

Pap, mam, Karin en Berly, jullie zijn er altijd voor me. Dank voor jullie onvoorwaardelijke steun. Wat geniet ik van alle familiemomentjes (met Rick, Sander, Tijn, Sam en Bente) en van de mijlpalen die we bereiken. Deze kunnen we ook afstrepen! 



\section{CURRICULUM VITAE}

Jeanny de Groot was born in Meerlo-Wanssum, the Netherlands on May 24, 1988. In 2005, she graduated from high school (Gymnasium, Dendron College, Horst) and started medical school at the Radboud University in Nijmegen. After graduating in 2012, she started working as a medical trainee at the Department of Gynecology and Obstetrics, Atrium Medical Center, Heerlen. From March 2013 untill June 2016 she worked as a $\mathrm{PhD}$ candidate at the Department of Family Medicine at Maastricht University under supervision of prof. dr. T. van der Weijden, dr. B.F.M. Slangen and dr. J.M.C. Maessen. During her PhD trajectory she worked on a national project to implement ERAS in gynecologic oncology. In 2016, she started her residency in gynecology and obstetrics at the Zuyderland Medical Center, Sittard - Heerlen, and at the Maastricht University Medical Center, Maastricht. She finished her PhD research project that resulted in this thesis during her residency. 



\section{LIST OF PUBLICATIONS}

J.J.A. de Groot, L.E.J.M. van Es, J.M.C. Maessen, C.H.C. Dejong, R.F.P.M. Kruitwagen, B.F.M. Slangen. Diffusion of Enhanced Recovery principles in gynecologic oncology surgery: is active implementation still necessary? Gynecol Oncol 2014;134: 570-5.

J.J.A. de Groot, J.M.C. Maessen, B.F.M. Slangen, B. Winkens, C.D. Dirksen, T. van der Weijden. A stepped strategy that aims at the nationwide implementation of the Enhanced Recovery After Surgery programme in major gynaecological surgery: study protocol of a cluster randomised controlled trial. Implement Sci 2015;10:106.

S.M.C. Ament, J.J.A. de Groot, J.M.C. Maessen, C.D. Dirksen, T. van der Weijden, J.M.P. Kleijnen. Sustainability of professionals' adherence to clinical practice guidelines in medical care: a systematic review. BMJ Open 2015; 5:e008073.

J.J.A. de Groot, S.M.C. Ament, J.M.C. Maessen, C.H.C. Dejong, J.M.P. Kleijnen, B.F.M. Slangen. Enhanced recovery pathways in abdominal gynecologic surgery: a systematic review and meta-analysis. Acta Obstet Gynecol Scand 2016; 95: 382-95.

J.J.A. de Groot, J.M.C. Maessen, C.H.C. Dejong, B. Winkens, R.F.P.M. Kruitwagen, B.F.M. Slangen, T. van der Weijden, all the members of the study group. Interdepartmental spread of innovations: a multicentre study of the enhanced recovery after surgery programme. World J Surg 2018; 42: 2348-2355.

J.J.A. de Groot, M. Timmermans, J.M.C. Maessen, B. Winkens, C.D. Dirksen, B.F.M. Slangen, T. van der Weijden, on behalf of the SINERGY nonparticipant group. Quality improvement strategies for organizational change: a multiphase observational study to increase insight into nonparticipating organizations. BMC Health Serv Res 2018;18: 1011.

J.J.A. de Groot, B.F.M. Slangen, J.M.C. Maessen, B. Winkens, C.D. Dirksen, T. van der Weijden, on behalf of all the members of the study group. Evaluating a tailored stepped implementation strategy on the improvement of perioperative care in gynecologic oncology. A cluster randomized clinical trial. (Submitted). 